Document downloaded from:

http://hdl.handle.net/10251/65421

This paper must be cited as:

Payri, R.; García Oliver, JM.; Xuan, T.; Bardi, M. (2015). A study on diesel spray tip penetration and radial expansion under reacting conditions. Applied Thermal Engineering. 90:619-629. doi:10.1016/j.applthermaleng.2015.07.042.

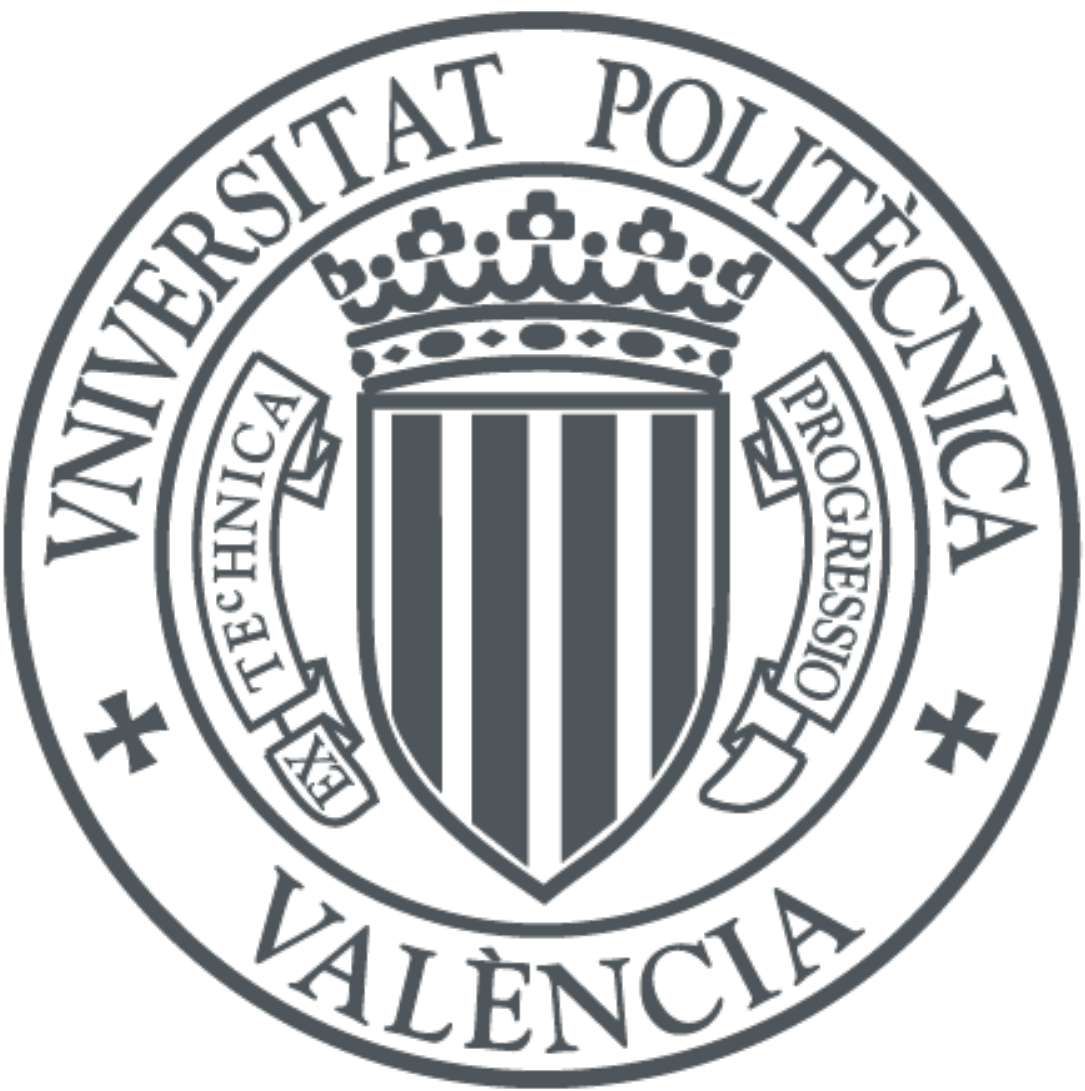

The final publication is available at

http://dx.doi.org/10.1016/j.applthermaleng.2015.07.042

Copyright Elsevier

Additional Information 


\section{Elsevier Editorial System(tm) for Applied Thermal Engineering Manuscript Draft}

Manuscript Number: ATE-2015-8432R2

Title: A study on Diesel spray tip penetration and radial expansion under reacting conditions

Article Type: Research Paper

Keywords: tip penetration; radial expansion; reacting spray; Schlieren imaging

Corresponding Author: Dr. Jose Maria Garcia-Oliver, Ph.D.

Corresponding Author's Institution: Universitat Politècnica de València

First Author: Raul Payri, PhD

Order of Authors: Raul Payri, PhD; Jose Maria Garcia-Oliver, Ph.D.; Tiemin Xuan; Michele Bardi, PhD

Abstract: The shape of Diesel spray was investigated at real engine conditions in a constant pressure combustion chamber. Schlieren imaging technique was used to make quantitative measurements of spray tip penetration and radial width stressing the impact that the fuel combustion and heat release have on the spray shape. The heat-release region and the Lift-off length were identified measuring $\mathrm{OH}^{*}$ chemiluminescence. The fuel (n-dodecane) as well as the operating conditions and the injector used (single axially oriented hole, $89 \mu \mathrm{m}$-diameter) were chosen following the indications by the Engine Combustion Network. The effects of different operating parameters on the axial and radial expansion were also investigated. According to the results the reacting spray can be divided into three parts: an inert part a transient part and a quasi-steady part that lays between the previous regions. A new method for evaluating this radial expansion of reacting spray was developed and the parameter was evaluated under the different operating conditions. The results show that the radial expansion increases with increasing injection pressure and decreasing ambient temperature and ambient density. The oxygen concentration has no obvious effect on the radial expansion. 


\title{
A study on Diesel spray tip penetration and radial expansion under reacting conditions
}

\author{
Raul. Payri, Jose.M. García-Oliver ( $\left.{ }^{\star}\right)$, Tiemin. Xuan \\ CMT Motores Térmicos - Universitat Politècnica de València, Valencia (Spain) \\ Michele. Bardi \\ LAV - Swiss Federal Institute of Technology, Zurich (Switzerland) \\ (*) Corresponding Author. Contact data: email: igarciao@mot.upv.es Tel: +34 963877650 \\ Camino de Vera s/n 46022 Valencia - Spain
}

\begin{abstract}
The shape of Diesel spray was investigated at real engine conditions in a constant pressure combustion chamber. Schlieren imaging technique was used to make quantitative measurements of spray tip penetration and radial width stressing the impact that the fuel combustion and heat release have on the spray shape. The heat-release region and the Lift-off length were identified measuring $\mathrm{OH}^{*}$ chemiluminescence. The fuel (n-dodecane) as well as the operating conditions and the injector used (single axially-oriented hole, $89 \mu \mathrm{m}$-diameter) were chosen following the guidelines of the Engine Combustion Network. The effects of different operating parameters on the axial and radial expansion were also investigated. According to the results the reacting spray can be divided into three parts: an inert part, a transient one, and a quasi-steady one that lays between the two other regions. A new method for evaluating this radial expansion of reacting spray was developed, which was evaluated under the different operating conditions. Results show that the radial expansion increases with increasing injection pressure and decreasing ambient temperature and ambient density. The oxygen concentration has no obvious effect on the radial expansion.
\end{abstract}

Keywords: tip penetration; radial expansion; reacting spray; Schlieren imaging

\section{Highlights:}

Spatial evolution of the reacting spray can be divided into three parts.

The radial contour of the reacting spray is the result of shifting the inert one by an approximately constant value

The radial expansion increases with higher injection pressure and lower ambient gas temperature.

The radius expansion decreases with higher ambient gas density.

Oxygen concentration has no significant effect on the radial expansion.

Nomenclature

ASOI After the start of injection ( $\mu \mathrm{s})$

CFD Computational Fluid Dynamics (-)

CPF Constant-pressure flow facility 
ECN Engine Combustion Network (-)

ICCD Intensified CCD sensor (-)

$\mathrm{k}_{\mathrm{r}}$ the slope of fitting line

LOL lift-off Length ( $\mathrm{mm}$ )

$\mathrm{O}_{2} \%$ Oxygen concentration (-)

$\mathrm{P}_{\mathrm{i}} \quad$ Injection pressure (bar)

$\overline{\Delta \mathrm{R}}$ the time-averaged radial difference of the quasi-steady part between reacting and inert spray contour $(\mathrm{mm})$

SOC Start of combutstion $(\mu \mathrm{s})$

$\mathrm{S}_{\mathrm{i}}$ Inert spray tip penetration $(\mathrm{mm})$

$\mathrm{S}_{\mathrm{r}}$ Reacting spray tip penetration $(\mathrm{mm})$

$\mathrm{T}_{\mathrm{a}}$ Ambient gas temperature $(\mathrm{K})$

ID Ignition delay $(\mu s)$

Greek symbols

$\mathrm{Pa}$ gas density $\left(\mathrm{kg} / \mathrm{m}^{3}\right)$

$\rho_{\mathrm{f}}$ fuel density $\left(\mathrm{kg} / \mathrm{m}^{3}\right)$

$\theta_{\mathrm{r}}$ Spreading angle of reacting spray

\section{Introduction}

Fuel-air mixing plays a significant role in combustion and emission process in diesel engine, which can be controlled by different injection parameters [1], injection strategies [2], advanced combustion modes[3] or fuel blending [4]. The complexity of the phenomena characterizing this process brought a significant challenge to the research community boosting the investigations in this field: experimental and computational tools have been significantly developed along the last decade and important goals have been achieved.

The definition and experimental characterization of many parameters related to specific spray features (tip penetration, spreading angle, liquid phase penetration, lift-off length etc) brought an important step forward in the understanding of the process [5][6][7]. Thanks to that, conceptual and numerical models have been developed to reproduce the whole Diesel injection process outlining the complex physical and chemical processes that occur behind the experimental observations [1][8].

Probably the most significant contribution in this direction are two conceptual models for conventional combustion and low temperature combustion based on different optical techniques proposed in [9] and [10], which give detail elaboration about how fuel-air mix in reacting conditions.

The increasing need for more accurate and predictive models together with the possibility of performing more detailed experiments still highlight some lack of knowledge. Few works in the literature address the effect that combustion has on the shape and mixing of the spray. Siebers's study [11] shows that the reacting spray evolution depends on the ambient density. The similar trend was also observed by Pickett and Hoogterp [12]. The spray penetration under reacting conditions was 
studied by Desantes et al. [13] and one defined spreading angle was applied for representing the radius expansion. In reference [14], the reacting and non-reacting spray penetration was also investigated with schileren technique. In most of such works, analysis is mainly based upon the effect of heat release on spray tip penetration by comparing the spray behavior when injected into an inert $(\mathrm{O} 2=0 \%)$ or a reacting $(\mathrm{O} 2>0 \%)$ atmosphere. Following a similar approach, the main goals of the present study are 1) to highlight the modification in the spray shape observed at reacting conditions 2) to investigate the mechanism behind these observations and 3) to provide quantitative data to the modelers that approach this problem.

This work is framed within the activities of the Engine Combustion Network (ECN): the ECN is a worldwide group of research institutes that started a collaboration to provide high quality data and consistent results to modelers [15]. The work of the group lays on several coordinated efforts in the Diesel research field: the complete definition of a standard reference condition called Spray A condition (and defining suggested parametric variations of the boundary conditions), the use of nominal identical injectors and the organic cross check of the data obtained by different facilities and through different techniques [16]. In this way, the results provided in this paper will also contribute to extend and integrate the open-access database provided by the community.

In the present work, the experiments were performed in a constant-pressure flow facility (CPF) able to reach 150 bar ambient pressure and $1,000 \mathrm{~K}$ ambient temperature. The optical diagnostics performed are:

- Schlieren imaging, to detect complete boundary of the inert and reacting spray

- $\mathrm{OH}^{*}$ chemiluminescence to measure lift-off length and to highlight the high-temperature heat release region of the reacting spray

The test matrix has been defined to isolate the effect of the combustion on the spray development: a set of tests was performed injecting the fuel in high temperature high pressure atmosphere but in pure Nitrogen. Then the set of tests have been repeated at the same thermodynamic conditions but increasing the oxygen content ( $15 \%$ and $21 \%$ (vol.)).

The analysis of the results focuses on the understanding of the impact that the combustion has on the modification of the spray boundaries defining also a criterion to quantify what will be defined afterward as radial expansion of the spray.

Including the present introduction, this document is composed of four sections. The next section gives a detailed description about the experimental apparatus, the setup of the two optical techniques, the different operating conditions which were investigated in this paper and the image processing. In the third section, the results under inert and reacting conditions were analyzed and discussed. The last section of the paper summarizes some of the most important conclusions of this investigation. 


\section{Experimental setup and Methodology}

\subsection{Experimental facility}

A constant-pressure flow (CPF) test chamber, as shown in Fig. 1, capable of reproducing typical incylinder thermo-dynamic conditions of a Diesel engine was used. Three wide quartz windows (120 $\mathrm{mm}$ diameter) enable a favorable optical access to the test section. Compared to other facilities this test rig has the unique feature of obtaining nearly quiescent and steady thermodynamic conditions within the chamber, providing thus an important reduction in the time required for the tests and higher accuracy in the control of the boundary conditions [17]. A separated cooling system was used for the injector: the temperature of the coolant was adjusted in order to maintain constant the temperature of the nozzle tip in the different test conditions. The calibration of the injector temperature control system was presented in [17]. The thermodynamic conditions within the chamber have been deeply characterized in [18] measuring the temperature at different positions with thermocouples of different diameters and accounting for needed the radiation correction [18]. A complete description of the facility is given in [17].

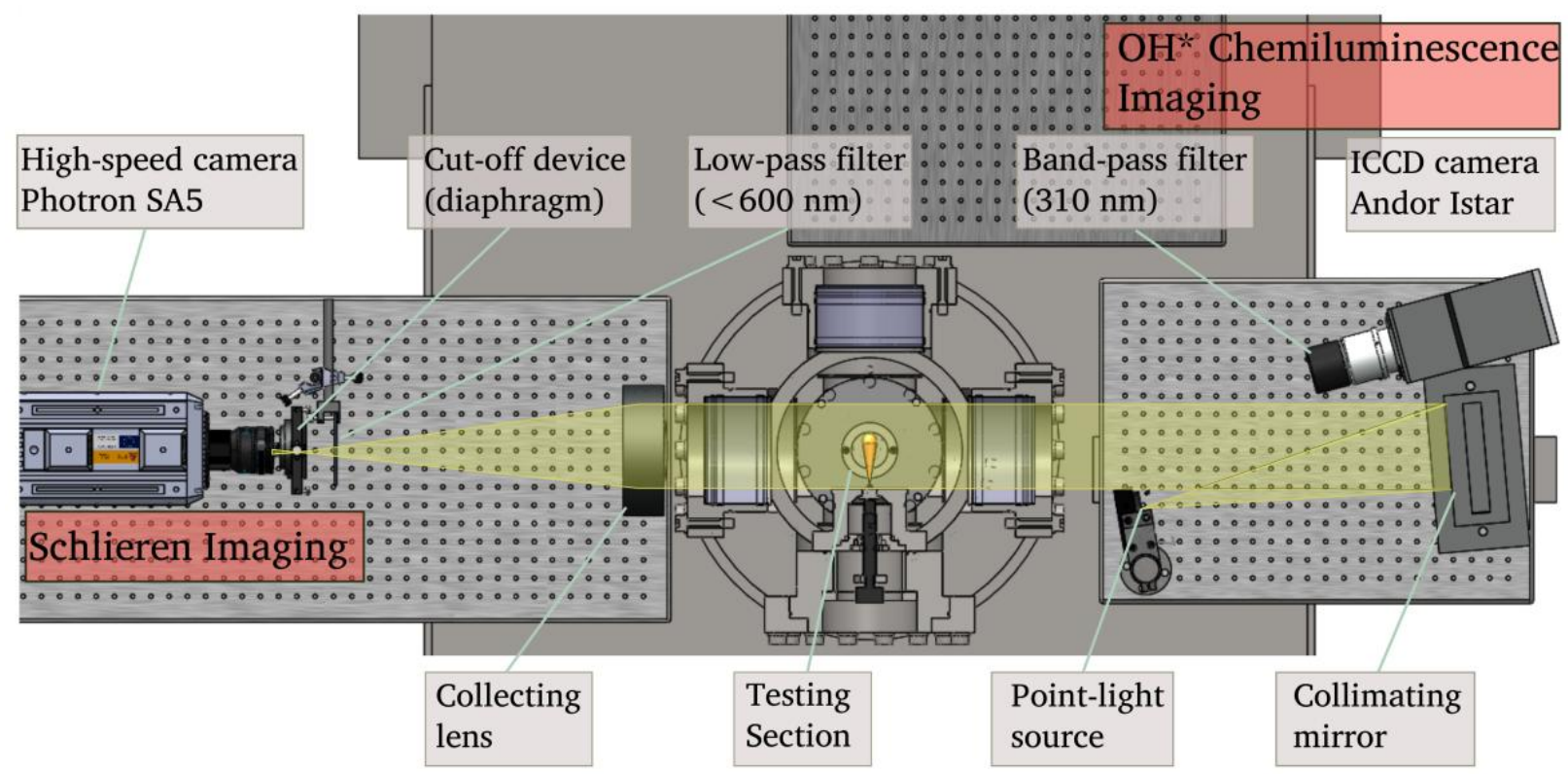

Fig. 1. Experimental layout: the test chamber is presented together with the optical setup.

\subsection{Test matrix}

The investigated operating conditions are summarized in Table 1 which is same as that in the reference [19] where the focus was on ignition and LOL, while here more details are given on the dynamics of the spray. The boundary conditions chosen aim at the reference Spray $A$ operating conditions ( $\left.T_{a}=900 \mathrm{~K}, \rho_{a}=22.8 \mathrm{~kg} / \mathrm{m}^{3}, P_{i}=1,500 \mathrm{bar}\right)$ and the parametric variation of the main variables as defined by ECN Working Group [16]. As previously mentioned an objective of the tests is the comparison of the spray contours measured at inert and reacting conditions. The parametric variation in the injection parameters (injection pressure) and thermodynamic conditions within the 
chamber (ambient temperature and ambient density) were performed by injecting the fuel into pure nitrogen (inert condition) or into an atmosphere with nitrogen and different percentages of oxygen (15, $21 \%$ (vol.)). In order to improve the statistical reliability of the results presented, 8 injection cycles were recorded for each operating condition.

Table 1. Operating conditions

\begin{tabular}{lll}
\hline Parameters & values & units \\
\hline Fuel & $\mathrm{n}$-Dodecane & - \\
Nozzle diameter & 89 & $\mu \mathrm{m}$ \\
Nozzle k-factor & 2.1 & - \\
Energizing time & 3500 & $\mu \mathrm{s}$ \\
Injection duration & 5200 & $\mu \mathrm{s}$ \\
Tip temperature & 390 & $\mathrm{~K}$ \\
Injection pressure $\left(\mathrm{P}_{\mathrm{i}}\right)$ & $500 ; 1,000 ; 1500$ & $\mathrm{bar}$ \\
Ambient gas temperature $\left(\mathrm{T}_{\mathrm{a}}\right)$ & $800 ; 850 ; 900$ & $\mathrm{~K}$ \\
Ambient density $\left(\rho_{\mathrm{a}}\right)$ & $15.2 ; 22.8$ & $\mathrm{~kg} / \mathrm{m}^{3}$ \\
Oxygen concentration $\left(\mathrm{O}_{2} \%\right)$ & $0 ; 15 ; 21$ & $\%(\mathrm{vol})$. \\
\hline
\end{tabular}

\subsection{Injection system}

The injection system employed consists of commercial available components: a high pressure volumetric Bosch CP3 pump driven by an electric motor; a common rail with pressure regulator controlled by a PID system.

The injector employed, based on second generation Common-Rail, is part of the ECN injector dataset (ref: \#210675 [15]). The injector features a single-hole axial nozzle enabling the advantage to focus on the fundamental behavior of the spray eliminating both the effects and the uncertainties related to the temperature boundary layer close to the walls and the spray-spray interaction. Moreover, ECN the internal geometry of the nozzle and the corresponding hydraulic behavior has been deeply characterized and the data are available online [15].

Following the ECN guidelines, a single-component fuel has been employed ( $n$-dodecane) to facilitate the comparison with CFD simulations.

\subsection{Optical techniques and processing methods}

Two imaging techniques were employed simultaneously in this study:

- High-speed Schlieren

- Time averaged $\mathrm{OH}^{*}$ chemiluminescence

The details of the measurements and of the setup employed are given in the following sections and the sketch of the experimental layout is presented in Fig. 1.

Table 2. Details of the optical setup used in the study.

\begin{tabular}{lcc}
\hline & $\begin{array}{c}\text { Schlieren } \\
\text { Imaging }\end{array}$ & $\mathrm{OH}^{*}$ chemilum. \\
\hline Camera & Photron SA-5 & Andor - Istar
\end{tabular}




\begin{tabular}{lcc} 
Sensor Type & CMOS & ICCD \\
Lens & $50 \mathrm{~mm}$ & $100 \mathrm{~mm}-$ U.V. \\
Diaphragm & $4 \mathrm{~mm}$ & - \\
Filter & - & $310 \mathrm{cwl} \pm 5$ \\
Frame Rate & $50 \mathrm{kfps}$ & 1 frame/injection \\
Shutter time & $4 \mu \mathrm{s}$ & $3 \mathrm{~ms}$ (from 2 to $5 \mathrm{~ms}$ ASOI) \\
Repetitions & 8 & 15 \\
Pixmm & 5.26 & 5.85 \\
\hline
\end{tabular}

\subsubsection{High speed Schlieren imaging}

A conventional Schlieren single-pass arrangement was employed to detect the complete spray boundaries at high temperature and high pressure conditions.

Schlieren imaging technique enables to detect gradients in the refractive index of a transparent medium [19]. The technique relies on the deviation of a light beam produced when light passes through non-homogeneous fluids. A Diesel spray injected at high temperature and high pressure conditions causes variations of refractive index gradient for three main reasons: a) the different optical properties between the fuel and the ambient gas b) the gradients in density /temperature that are generated within the spray related to the mixing process $\mathrm{c}$ ) the heat released during the combustion.

As a consequence, images acquired show the spray boundaries including liquid and vapor phase fuel, and, under reacting conditions, the regions which contain combustion products.

The Schlieren setup employed (Fig. 1) includes different components: Xe-Arc lamp and a $1 \mathrm{~mm}$ diameter pin-hole constituting the point light source of the system; a parabolic mirror used to collimate the light from the pin-hole in a parallel beam; a collecting lens to focus the parallel beam in a single point and a high-speed camera(50 kfps). A diaphragm (4 mm aperture) was used as a cut-off device to enhance the sensitivity of the setup while a short-pass filter $(<600 \mathrm{~nm})$ was filtering the light beam to reject part of the soot incandescence light emissions. More details of the setup are indicated in Table 2.

The images captured in the tests were processed following an intensity-based criterion and applying a dynamic background subtraction. The methodology, described in details in the work by Benajes et al. [19], proved to be a robust one and provides binarized images of the spray region.

From each single spray image a binarization process results in a corresponding contour (Fig. 2) and a spray tip penetration. Following this processing step, a statistical analysis makes it possible to derive the following parameters:

- Average spray tip penetration (s) by averaging of the corresponding sample of repetitions for each time step.

- An average spray boundary for a given time step, which is obtained by calculation of spray probability maps from the corresponding sample of binarized images (Fig. 2). Such maps indicate the probability of finding a spray at one position. The average spray boundary is defined as the border of the region where the probability is equal to or higher than $50 \%$. Moreover, considering the spray axisymmetric, the spray contour was summarized in only two variables: axial position, and spray width. 
Schlieren images

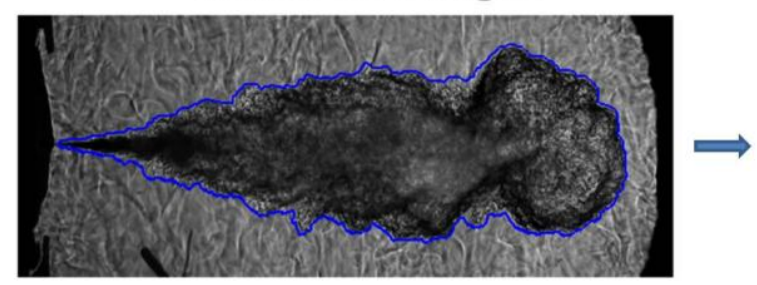

Binarization

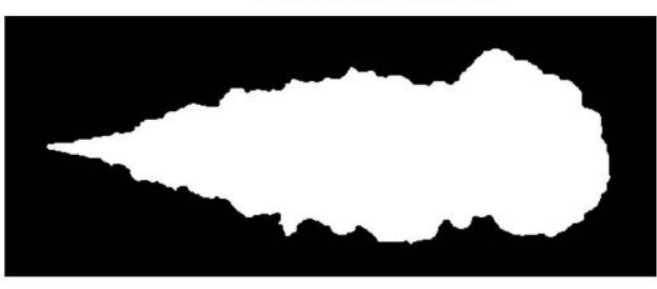

Rep.

\#1

\#8

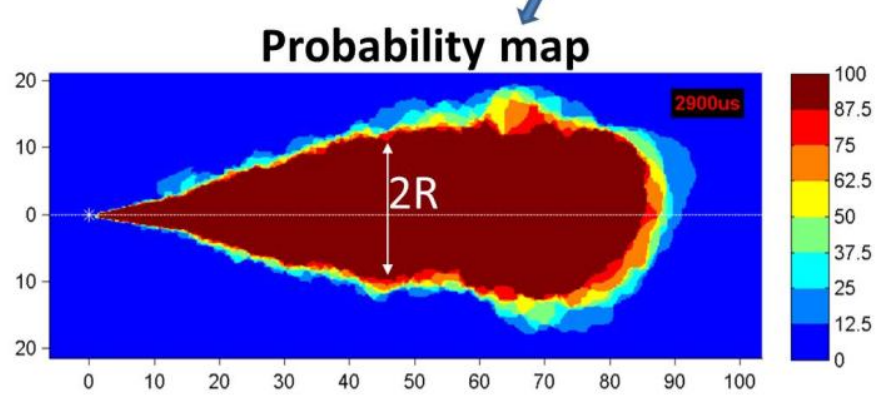

Fig. 2. The image processing workflow for Schlieren-derived images

\subsubsection{Time averaged $\mathrm{OH}^{*}$ chemiluminescence}

The high-temperature heat release region of a Diesel spray is generally related with the light emissions by the $\mathrm{OH}^{*}$ radicals decaying at their ground state. These light emissions have an important peak at $309 \mathrm{~nm}$ [19]. An ICCD camera (Andor I-star) fitted with a $100 \mathrm{~mm} \mathrm{f} / 2.8 \mathrm{UV}$ lens and a $310 \pm 5 \mathrm{~nm}$ interferometric filter was used with a constant intensifier gating time widow synchronized with the injection between 2.0 and $5.0 \mathrm{~ms}$ after the start of the injections (ASOI): in this way the steady part of the combustion process was averaged along the injection event and the shot-to-shot deviation was reduced. The $\mathrm{OH}^{*}$ chemiluminescence images were employed for the calculation of the Lift-off length. The procedure followed and the results have been already presented in [19].

\section{Results and discussion}

\subsection{Reference analysis at Spray A conditions}

Taking into account the limitations of the optical access of the CPF chamber and the frequency of the cases used for comparison in later sections, the baseline Spray A condition was selected for the reference analysis in this section. Because of the early ignition caused by high ambient temperature and high density and fast spray development created by high injection velocity, the full development period of the spray can be recorded by the camera before the end of injection.

Fig. 3 and Fig. 4 (right) show the temporal evolution of the tip penetration and radial width of inert and reacting sprays under Spray A condition, respectively. In these figures as well as in the subsequent ones presented in this paper, the time is referred to the start of the injection with the acronym ASOI (After Start Of Injection). Sample images from a single injection cycle are shown in Fig. 4 (left). As shown in top plot of Fig.3, the red and the blue solid line represent the average spray penetration from the eight repetitions under reacting and inert conditions, respectively. Due to the very 
small difference in molecular weight between oxygen and nitrogen, ambient pressure is essentially the same for the nominal ambient density and temperature values. The vertical red dashed line in Fig. 3 represents the start of combustion obtained from Schlieren images following the methodology in [20]. Both inert and reacting spray tip penetrations increase steadily with time, but the reacting case shows an apparent acceleration after the start of combustion. To further analyze this behavior, the bottom plot shows the penetration ratio (i.e. reacting divided by inert penetration at each time step), consistently with the analysis carried out in a previous work from Desantes et al [13] for n-heptane sprays under similar operating conditions.As described in that paper, the temporal evolution of the reacting spray can be divided into five stages:

I.Non-reacting phase: from the start of injection to the start of combustion, where the penetration ratio should be essentially one. Results in Fig.3 shows values lower than one. This is due to the small penetration values, so that scattering between inert and reacting tests may result in a relatively important deviations of the ratio from one. .

II.Auto-ignition expansion phase: It is a relatively short phase marked by a first short-lived peak in penetration ratio. It spans from the start of combustion (SOC) to the time when the penetration between inert and reacting condition become the same again.

III.Stabilization phase: from the end of the previous stage to the time when the tip penetration starts to separate, as shown in [13] the duration of this phase depends a lot on the operating condition, under less reacting conditions this phase becomes longer. Under Spray A conditions this phase is almost non-existent.

IV.Acceleration phase: where the penetration ratio starts increasing with time.

V.Quasi-steady phase: where the tip penetration ratio reaches a relatively steady value.

There is a decrease of the penetration ratio after the time exceeds $4 \mathrm{~ms}$ because the reacting case reaches the limit of the optical access, as shown in the top plot in Fig.3. 


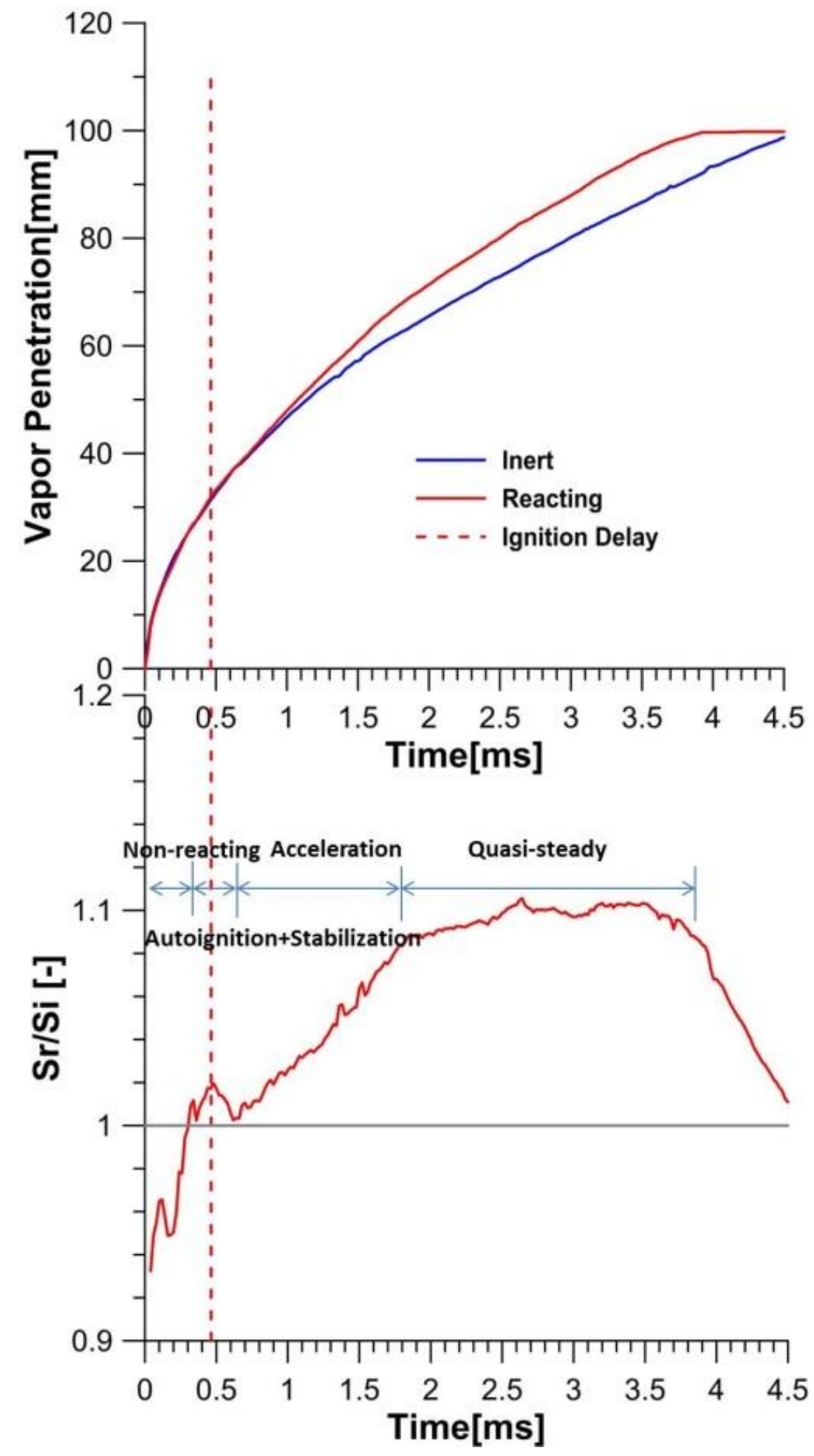

Fig. 3. Temporal evolution of the penetration and ignition delay (dashed line) from Schlieren image processing for both inert and reacting conditions (top) and corresponding penetration ratio(bottom). (Spray A condition)

The image sequence on the left hand side of Fig. 4 presents the temporal evolution of half images of a single injection for the reacting nominal Spray A condition. As mentioned above, the optical access was limited to $100 \mathrm{~mm}$ and the black area on the right top of these images is the window limit. As for the right image sequence of Fig. 4, the temporal evolution of the spray half-width (i.e. the spray radius) as derived from the probability images under reacting and inert conditions are 
shown here for the indicated timings. Differences between left and right image sequences of Fig. 4, are due to the comparison of average contours to instantaneous realizations of the experiment. The vertical red dashed line represents the average lift-off length as obtained from $\mathrm{OH}^{*}$ chemiluminescence images.

At $320 \mu \mathrm{s}$ ASOI, the spray behaves as an inert spray which corresponds to the non-reacting phase of the penetration mentioned above and there is no expansion neither in axial nor in radial direction. The contours of inert and reacting conditions still keep similar at $400 \mu \mathrm{s} \mathrm{ASOI}$, even though some part of the spray tip starts getting transparent as a consequence of the onset of cool flames. At about $450 \mu \mathrm{s}$ ASOI, the ignition takes place which directly leads to a separation of the penetration and radial expansion in the front part of the spray. This agrees with the auto-ignition expansion phase observed in the penetration plot. At $660 \mu \mathrm{s}$ ASOI, there is an obvious radial expansion for the reacting spray, and the tip penetration of both conditions become similar again. According to the momentum conservation, the strong increase in radial width leads to a slower penetration for the. This image

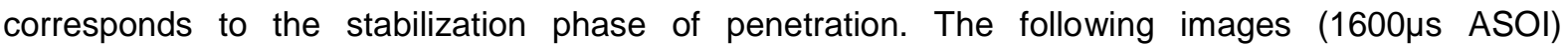
correspond to the acceleration phase of the penetration evolution. It can be seen that the initial radial expansion part downstream of the lift-off length is stabilized compared with the same part of the next plot $(2900 \mu \mathrm{s}$ ASOI), with a spray slightly wider than under inert conditions. At $1600 \mu \mathrm{s}$ ASOI a 'waist' part was found at around $60 \mathrm{~mm}$ from the nozzle where the reacting spray contour trends to be almost flat and overlaps with the inert spray contour. The last images (2,900us ASOI) correspond to the quasi-steady phase of the penetration. It was found that the stabilized part spans from the nozzle up to around $50 \mathrm{~mm}$. An apparent vortex came up after the jet head (left image), which can also be seen one the contour derived from the probability, which means that the 'waist' is statistically significative. This confirms that the transient tip of the spray is governed by a vortex with a very repetitive pattern. This phenomenon could also be found over a wide range of operating conditions which should be attributed to rapid gas entrainment [21]. 

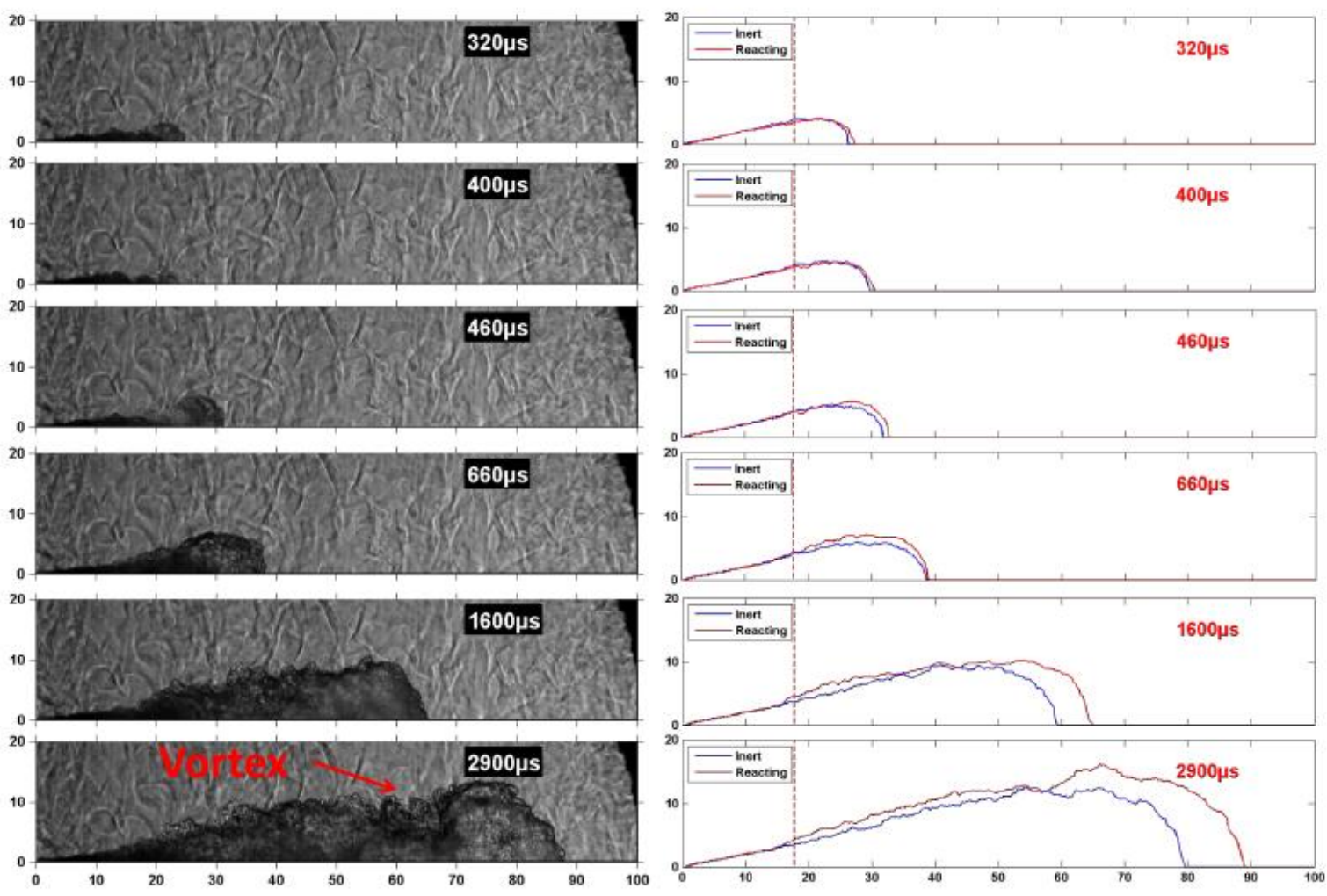

Fig. 4 Temporal evolution of the reacting spray contour obtained with Schlieren (left) and radial width both under inert and reacting condition(right) (spray A condition)

\subsection{Analysis of spatial evolution of reacting spray contour under baseline Spray A conditions}

Different approaches can be found in the literature to assess the radial dispersion of inert and reacting spray and many definitions for the spray spreading angle are given [5][13][22]. However, it was found that, especially at reacting conditions, the geometry of diesel spray is much more complicated than a cone shape and it was not well represented by a single parameter. In order to better understand the radial dispersion of the reacting spray, the radial widths for both inert and reacting spray under Spray A condition were compared. As shown in Fig.5, the red and blue solid lines represent the radius width of the reacting and inert spray separately. The red vertical dashed line represents the lift-off length as derived from $\mathrm{OH}^{*}$ chemiluminiscence images. It must be noted that the comparison of contours is performed at iso-spray tip penetration, not at the same timing. This means that while the time of inert spray is at $4380 \mu \mathrm{s}$ ASOI and the time of reacting spray is at $3620 \mu \mathrm{s}$ ASOI.

According to several experimental evidences, the structure of the reacting spray can be divided into 3 parts as shown in Fig. 3:

I.Quasi-steady Inert part, which is defined from the nozzle tip to the Lift-off length. Because of the absence of heat release, in this zone the spray behaves as an inert flow.

II.Quasi-steady reacting part, which is defined from lift-off length to the position where the contour stops increasing radially. In this part, the heat released by the oxidation reactions cause an increase in the radial width at every position of the spray axis. After a first 
transient where the radial width increases with the axial position, the difference remains almost constant throughout the region.

III.Transient part, which is defined from the end of the quasi-steady part to the spray tip. This part is much more complicated than other two ones, due to its transient nature. In the same way as for an inert spray, a reacting one is made up of a quasi-steady part (I and II) led by a transient tip. As sketched in Fig. 5, for a developed spray the extent of the steady part (I and II) is roughly $50 \%$ of the total spray tip penetration, compared to around $60 \%$ which is a reference value for the inert case, and agrees with the contour in Fig. 5.

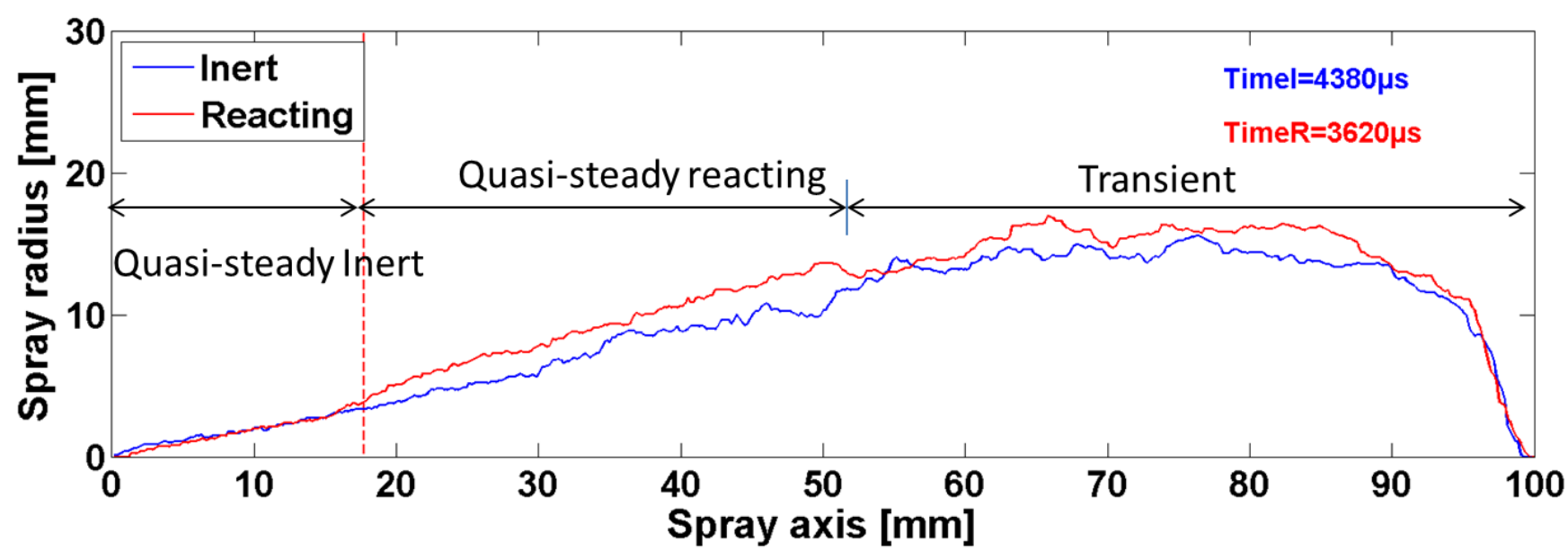

Fig. 5. Radial width for both inert and reacting spray under Spray $A$ condition $\left(S_{r}=97 \mathrm{~mm}, S_{i}=97 \mathrm{~mm}\right)$.

One of the aims of this paper is to gain understanding on the radial expansion characterizing the quasi-steady reacting region. To this end, it is necessary to define the position of the transition point from quasi-steady reacting part to the transient part. Two fitting lines to the inert and reacting spray contours were done by means of a least-squares algorithm from a distance to the nozzle $10 \%$ longer than the lift-off length to three different percentages $(40 \%, 50 \%, 60 \%)$ of the tip penetration. Fig. 6 shows the fitting lines with the contours of the three percentages at the same time. It is quite apparent that the $40 \%$ limit can be further extended downstream while still being valid, whereas the $60 \%$ case already runs into the transient tip. Accordingly, the lower limit of the transient tip will be defined hereafter as $50 \%$ of the tip penetration. 

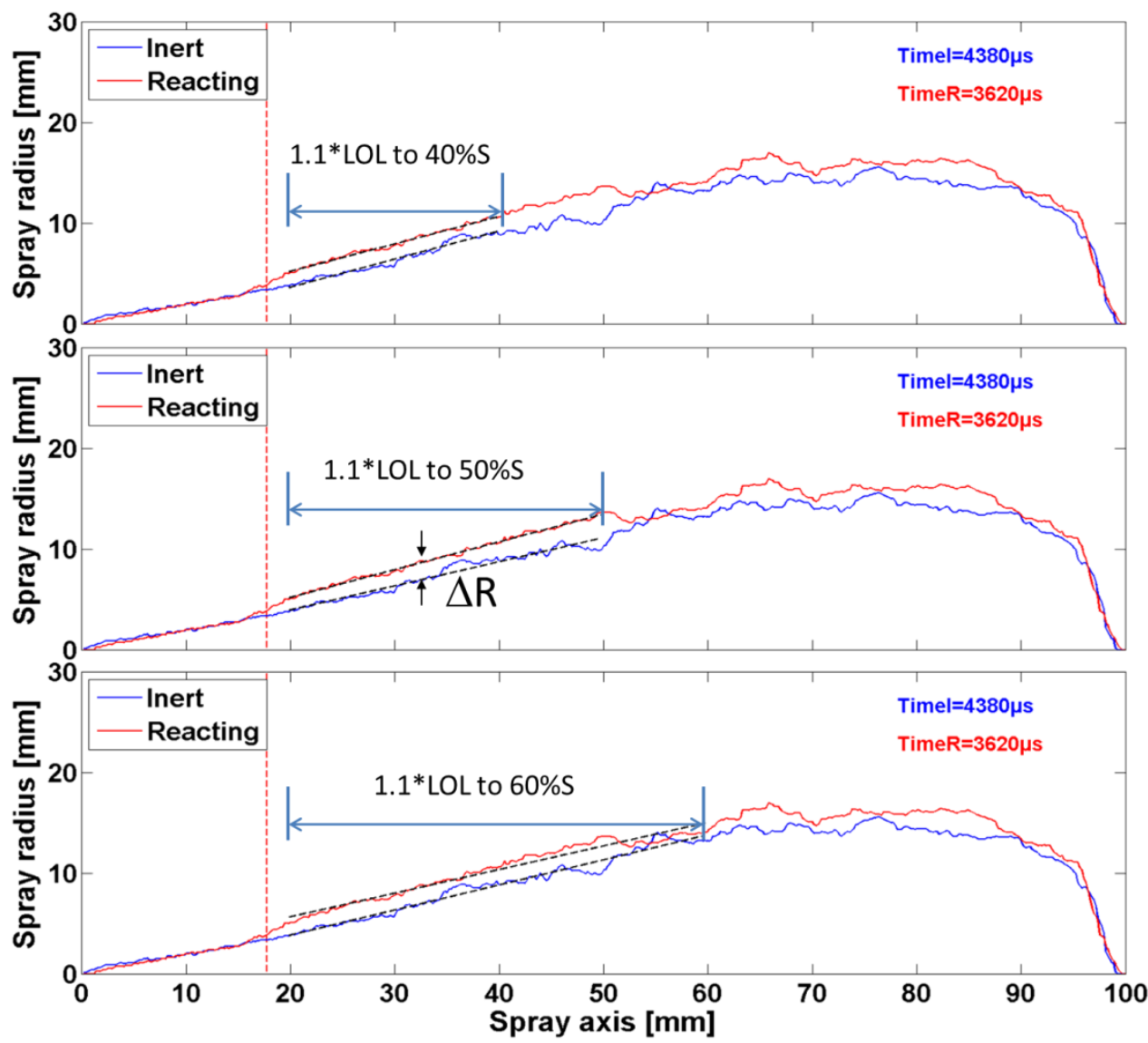

Fig.6. The fitting line of the inert and reacting spray at Spray A condition

From the derived contours, both inert and reacting spray cone angles were calculated according to the slope of the fitting line.

$$
\tan \left(\theta_{r} / 2\right)=k_{y}
$$

where $\theta_{r}$ is the spreading angle of the spray, $k_{r}$ is the slope of the fitting line.

The plots in Fig. 6 correspond to a single time instant. The corresponding time-resolved evolution of the spray angle under inert and reacting conditions is presented in Fig.6.

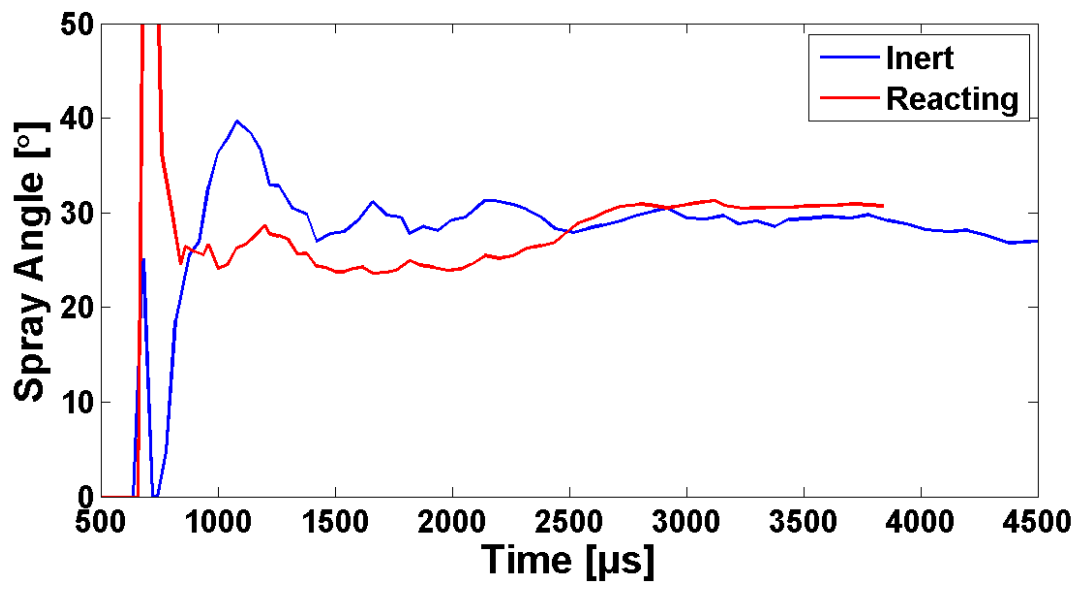

Fig. 7. The spread angle of the inert and reacting spray at Spray A condition 
Results show that both inert and reacting angles trend to be the same value after some time interval, i.e. the two fitting lines become parallel. This means that the radial expansion at different axis position of the quasi-steady reacting part is similar, in other words, the radial contour of the reacting spray is simply the result of shifting the inert one by an approximately constant value.

Based on the previous analysis, the radial increase should adequately describe the combustioninduced radial expansion along the whole spray axis. In order to find a single parameter to evaluate the radial expansion of the quasi-steady reacting part, the average value of the radial difference between inert and reacting conditions at every -time instant from a distance $10 \%$ longer than LOL to $50 \%$ of the spray tip has been calculated. After that, this spatially-averaged value was also timeaveraged into the parameter $\overline{\Delta \mathrm{R}}$. Because the reacting spray has not reached a stable states at the onset ignition period and the strong tip vortex has some influence on definition of this part, the time window for calculating the $\overline{\mathrm{AR}}$ was chosen from $1 \mathrm{~ms}$ to $2 \mathrm{~ms}$ after ignition delay. It has been observed that the defined parameter is stable in this time window for all cases except the low density case. One possibility for such a behavior is that the flame under low density condition is not as stable as that under high density. This quantitative indicator $\overline{\Delta \mathrm{R}}$ will be used in the following section to evaluate the expansion of the spray under parametric studies.

\subsection{Effect of operating parameters on reacting spray evolution}

\subsubsection{Effect of injection pressure and ambient gas temperature}

The temporal evolution of the effect of injection pressure on the penetration ratio and radius difference is shown in Fig. 8 The solid and dashed lines represent the penetration ratio and radius increase, respectively. The time window applied for calculating the time-averaged $\overline{\mathrm{AR}}$ was marked by the shadowed area in Fig.8 It can be observed that the penetration ratio at the quasi-steady phase increased with lower injection pressure This trend is not consistent with previous work [12], where the penetration ratio is shown to be similar during the final quasi-steady phase for different injection pressures, although n-heptane was used as a fuel, which is less reactive than n-dodecane. Regarding the radius width difference $(\Delta \mathrm{R})$, it keeps stable during the averaging time window and it eventually increases, which is probably caused by the strong vortex behind the jet head. The onset time of the quasi-steady phase of the penetration ratio is shown to have no relationship with the stable period of the radial difference. Comparing both penetration ratio and radius increase it can be concluded that the penetration ratio at the quasi-steady phase decreases with higher radial expansion. 


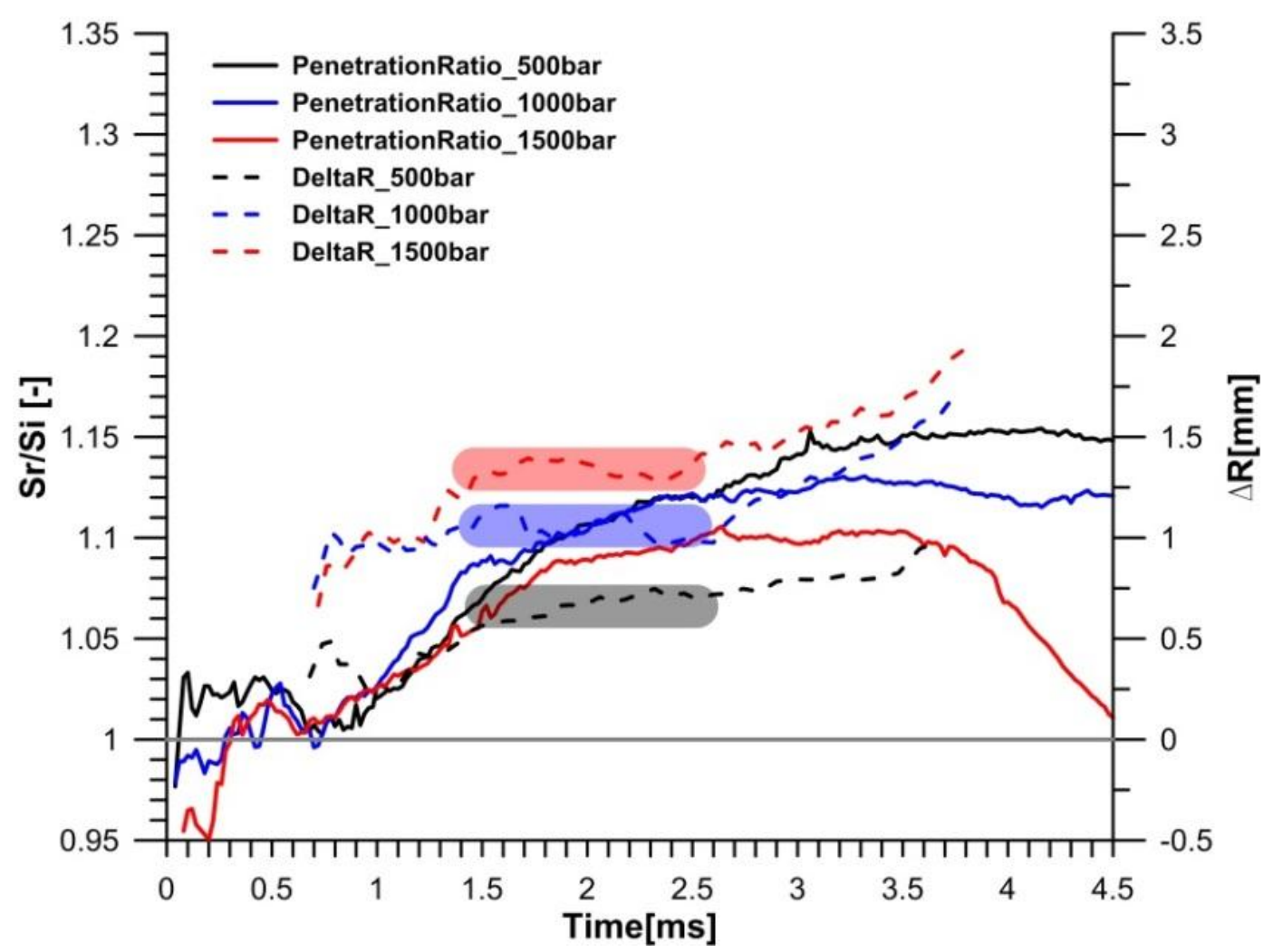

Fig.8. Temporal evolution of effects of injection pressure on penetration ratio and radius difference. $T_{a}=900 \mathrm{~K}, \rho_{a}=22.8 \mathrm{~kg} / \mathrm{m}^{3}, O_{2} \%=15 \%$.

On the other hand, Fig.9 shows that the temperature has a large effect both on the penetration ratio and radial expansion. With decreasing ambient temperature, the spray has a later ignition and a longer $\mathrm{LOL}$ obtained from $\mathrm{OH}^{*}$ chemiluminescence resulting in a leaner combustion. In terms of the spray penetration ratio, a longer duration of the stabilization phase is observed at lower ambient temperature, and most of the time along this phase the reacting spray tip penetrates slower than the inert one. As for the acceleration phase, the increasing rate and the peak value of the penetration ratio are similar for $850 \mathrm{~K}$ and $900 \mathrm{~K}$ temperature cases, while at $800 \mathrm{~K}$ both are lower.

As for the radial expansion, the value during the stable stage shows an increase with lower ambient temperature. Except the effect of vortex, a second factor, contributing to an increase on the radial difference at the later period of the evolution is the ending of the injection energizing after $3.5 \mathrm{~ms}$, although it is probably less significant. 


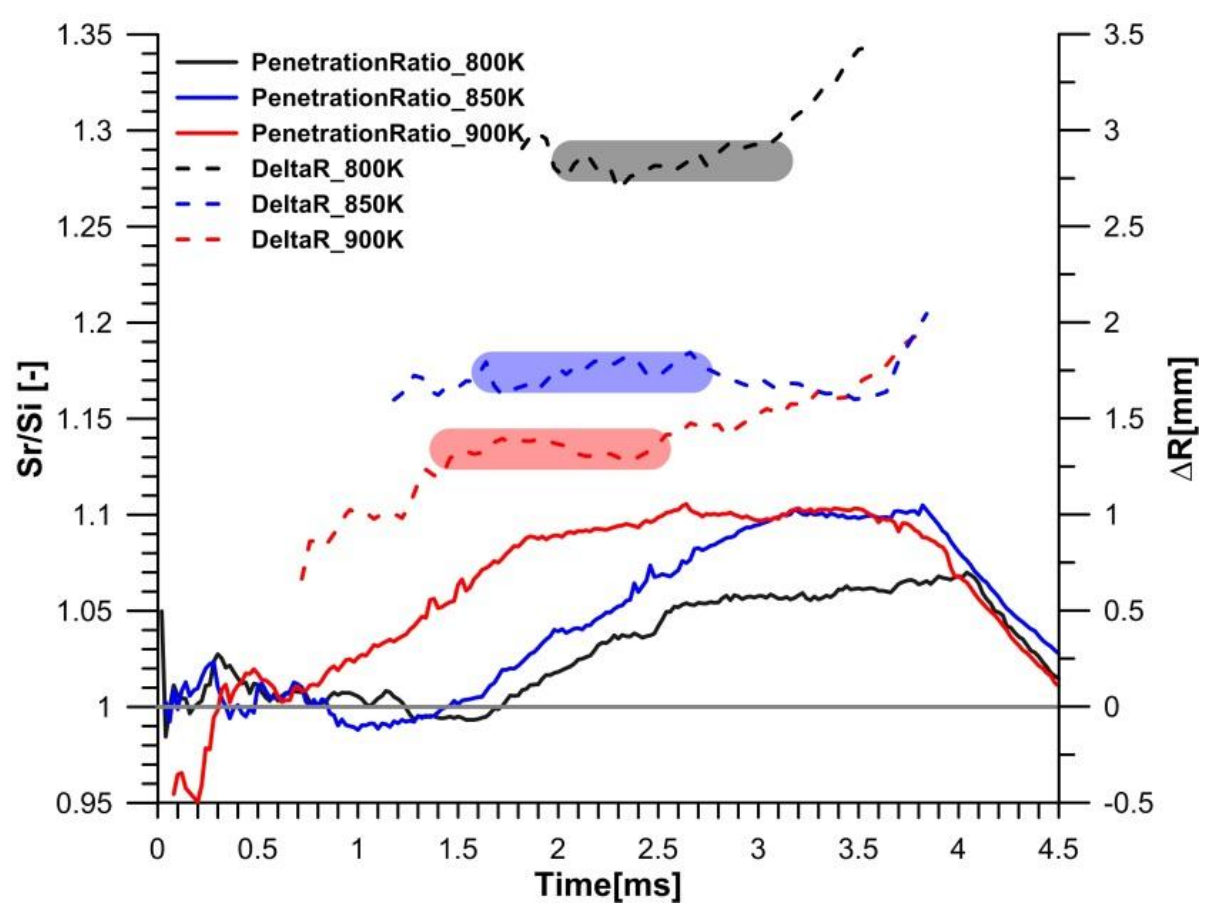

Fig.9. Temporal evolution of effects of ambient temperature on penetration ratio and radius difference. $P_{i=1,500 \mathrm{bar},} \rho_{a}=22.8 \mathrm{~kg} / \mathrm{m}^{3}, O_{2} \%=15 \%$.

The influence of injection pressure on the time-averaged $\overline{\Delta \mathrm{R}}$ at the three different temperatures is shown in Fig.10 The $\overline{\Delta \mathrm{R}}$ shows an increasing trend with higher injection pressure, the sensitivity is reduced with higher temperature. On the other hand, the plot also indicates that the temperature has a strong effect on $\overline{\Delta \mathrm{R}}$, especially under higher injection pressure.

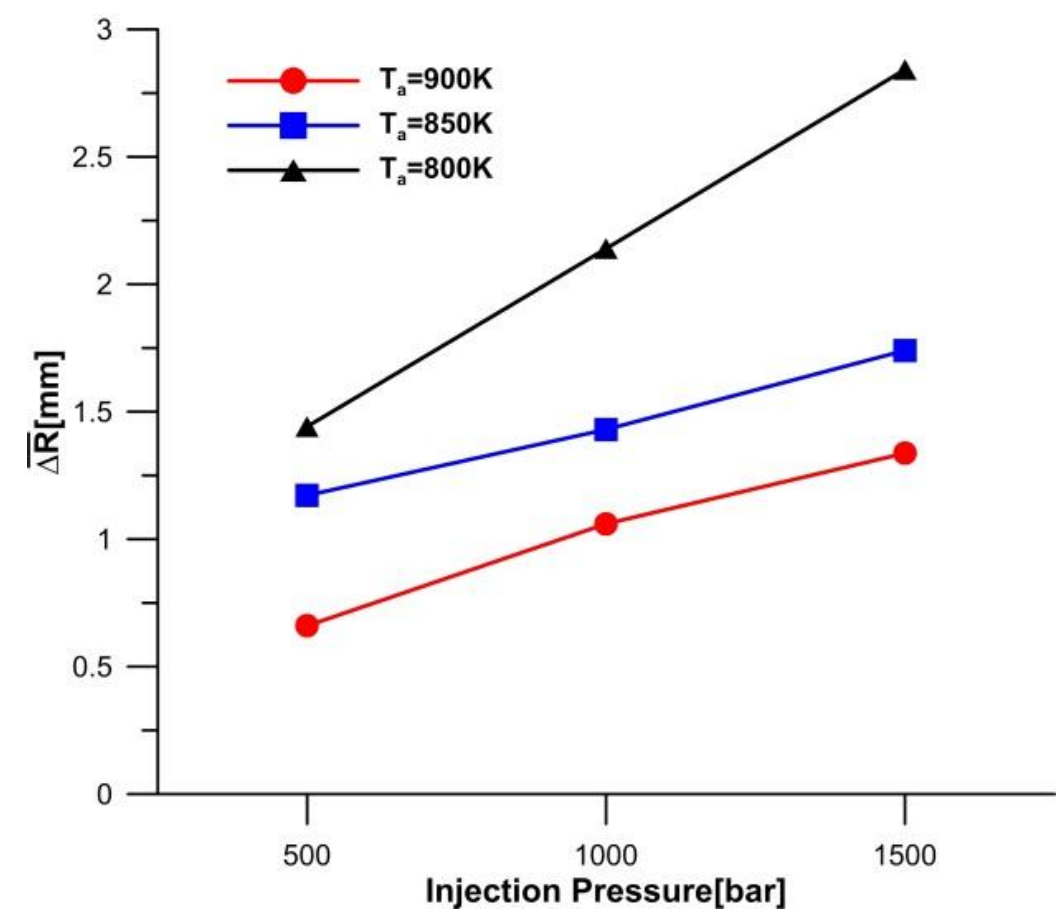

Fig.10. $\overline{\Delta \mathrm{R}}$ for parametric variation of injection pressure and ambient temperature. $\rho_{a}=22.8 \mathrm{~kg} / \mathrm{m}^{3}$, $O_{2} \%=15 \%$. 


\subsubsection{Effect of ambient density}

The temporal evolution of the penetration ratio and radial expansion when changing ambient density $\left(P_{i}=1,000\right.$ bar, $\left.T_{a}=900 \mathrm{~K}, O_{2} \%=15 \%\right)$ is shown in Fig.11 (left). There is an obvious increase in ignition delay and LOL with decreasing density. Thus, under the lowest density condition $\left(\rho_{a}=7.6\right.$ $\mathrm{kg} / \mathrm{m}^{3}$ ), a faster penetration and much longer ignition delay are observed, which prevents the observation of the acceleration and the quasi-steady phases because of the limitation of the optical access. It can be seen that the stabilization phase lasts longer with lower ambient density. At the quasi-steady phase, the penetration ratio increases with increasing density, which is different with previous work [12]. As for the radial difference, the observed evolution at $15.2 \mathrm{~kg} / \mathrm{m}^{3}$ condition is not as steady as for the other cases. This phenomenon can also be found when the injection pressure is at 1500 bar, which is not shown here. Probably, this was due to a more unstable flame development under low density condition.

The influence of the ambient density at two different injection pressures on the time-averaged $\overline{\Delta R}$ is shown in Fig.11 (right). As already commented, the time-averaged radial difference could not be obtained at $7.6 \mathrm{~kg} / \mathrm{m} 3$ because of the large LOL and ignition delay. As shown in Fig.11, the radial expansion decreases with higher ambient density, which is more sensitive at higher injection pressure
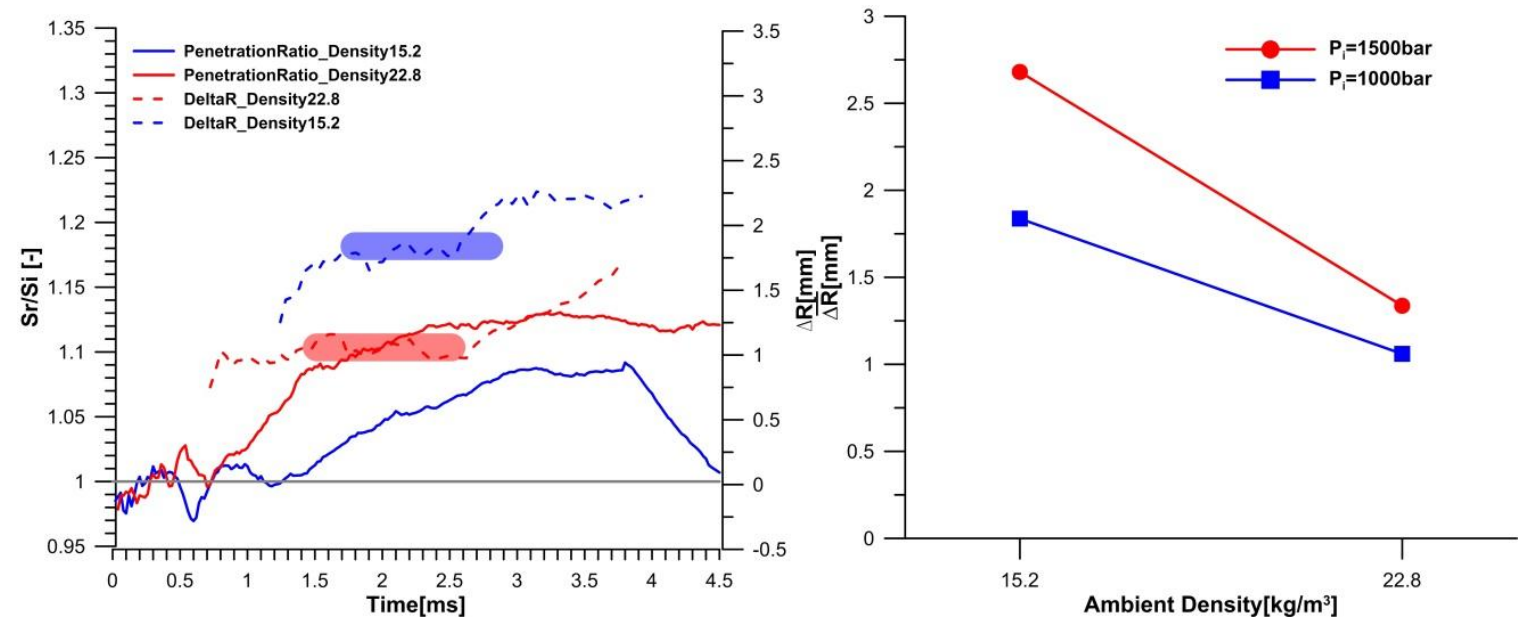

Fig.11. Temporal evolution of penetration ratio and radius expansion (left) and $\overline{\Delta R}$ (right) for a parametric variation of ambient density. $T_{a}=900 \mathrm{~K}, O_{2} \%=15 \%$.

\subsubsection{Effect of oxygen concentration}

Temporal evolution of the penetration ratio and radial expansion for an oxygen variation were investigated when keeping other parameters constant $\left(P_{l}=1,500 \mathrm{bar}, T_{a}=900 \mathrm{~K}, \rho_{a}=22.8 \mathrm{~kg} / \mathrm{m}^{3}\right)$. As shown in Fig.12 (left), when the oxygen concentration is $21 \%$, there is a sharp increase of the penetration ratio during the auto-ignition phase, as a consequence of the faster developing combustion process. The increasing rate and the peak value of the penetration ratio at the acceleration and quasi-steady phase always increase with higher oxygen concentration. However, the influence of oxygen on the radial expansion is not obvious, as shown in the temporal evolution of the radial difference (left) and the time-averaged $\overline{\Delta \mathrm{R}}$ (right). 

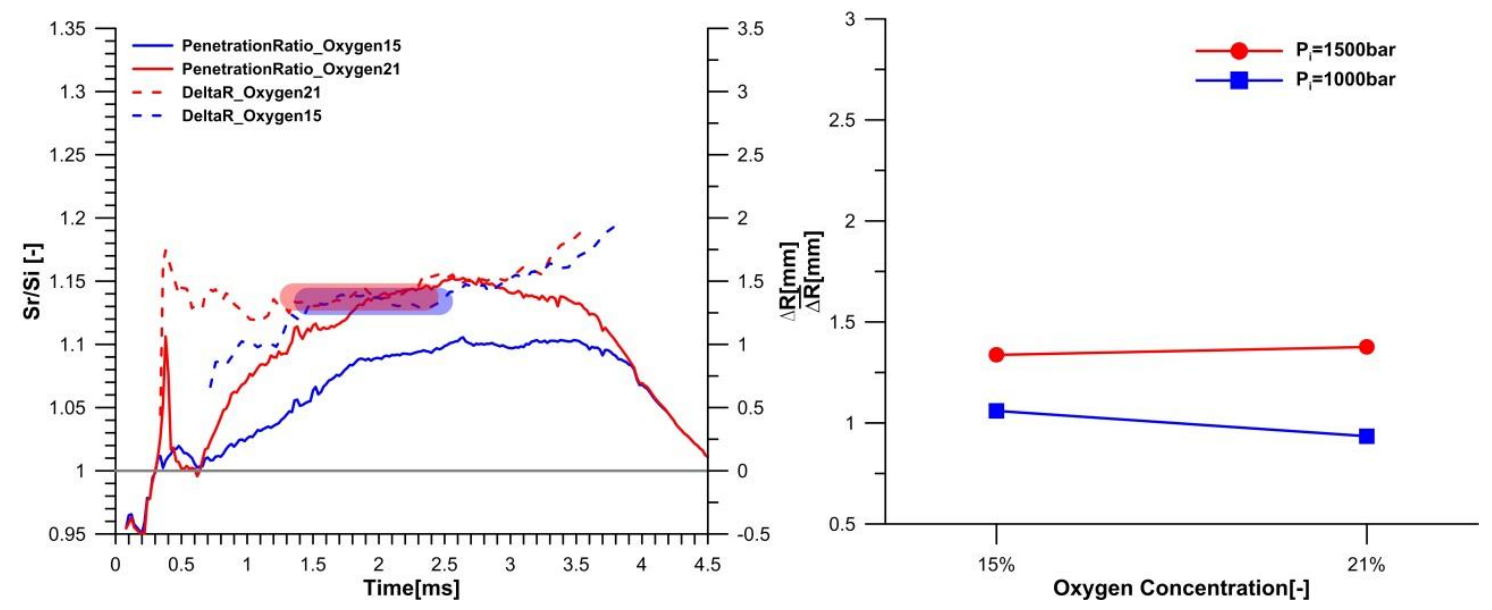

Fig.12. Temporal evolution of penetration ratio and radius expansion (left) and $\overline{\Delta R}$ (right) for a parametric variation of ambient density. $T_{a}=900 \mathrm{~K}, \rho_{a}=22.8 \mathrm{~kg} / \mathrm{m}^{3}$.

\subsubsection{Discussion}

From theprevious sections, two conclusions can be derived. First, there seems to be an inverse relationship between the radial increase and the penetration ratio. This has been observed for parametric variations of injection pressure, temperature and density. Only for the oxygen concentration is this relationship not observed. This can be explained to some extent in terms of simple momentum flux balances. From the point of view of a quasi-steady spray evolution, momentum flux at any cross section should be the same under inert or reacting conditions, and equal to the nozzle momentum flux. If by changing a parametric variable the radial increase is higher, the spray cross section is also higher, and therefore the expected average velocity should be lower, in agreement with the tip penetration ratio decrease. This argument is valid as long as density changes induced by transition to combustion are not very different among different operating conditions.

According to the previous reasoning, it would be important to evaluate which are the dependencies of the radius increase with the operating variables. The hypothesis has been made that the more fuel is available and therefore burnt at initial combustion stages, a stronger heat release and subsequent a higher lateral expansion is obtained.

Following this idea, the fuel mass downstream the lift-off length at the start of combustion (Mf) has been calculated for all experimental cases by means of 1D spray model [23][24]. The results are compared in Fig.13 with the radial increase. The blue points are the results of injection pressure and ambient temperature sweep, while the red and black points are the results of high oxygen concentration and low density cases respectively. According to this plot and the results in the above sections, it is quite apparent that a relationship exists between both variables, which to some extent validates the initial hypothesis. 


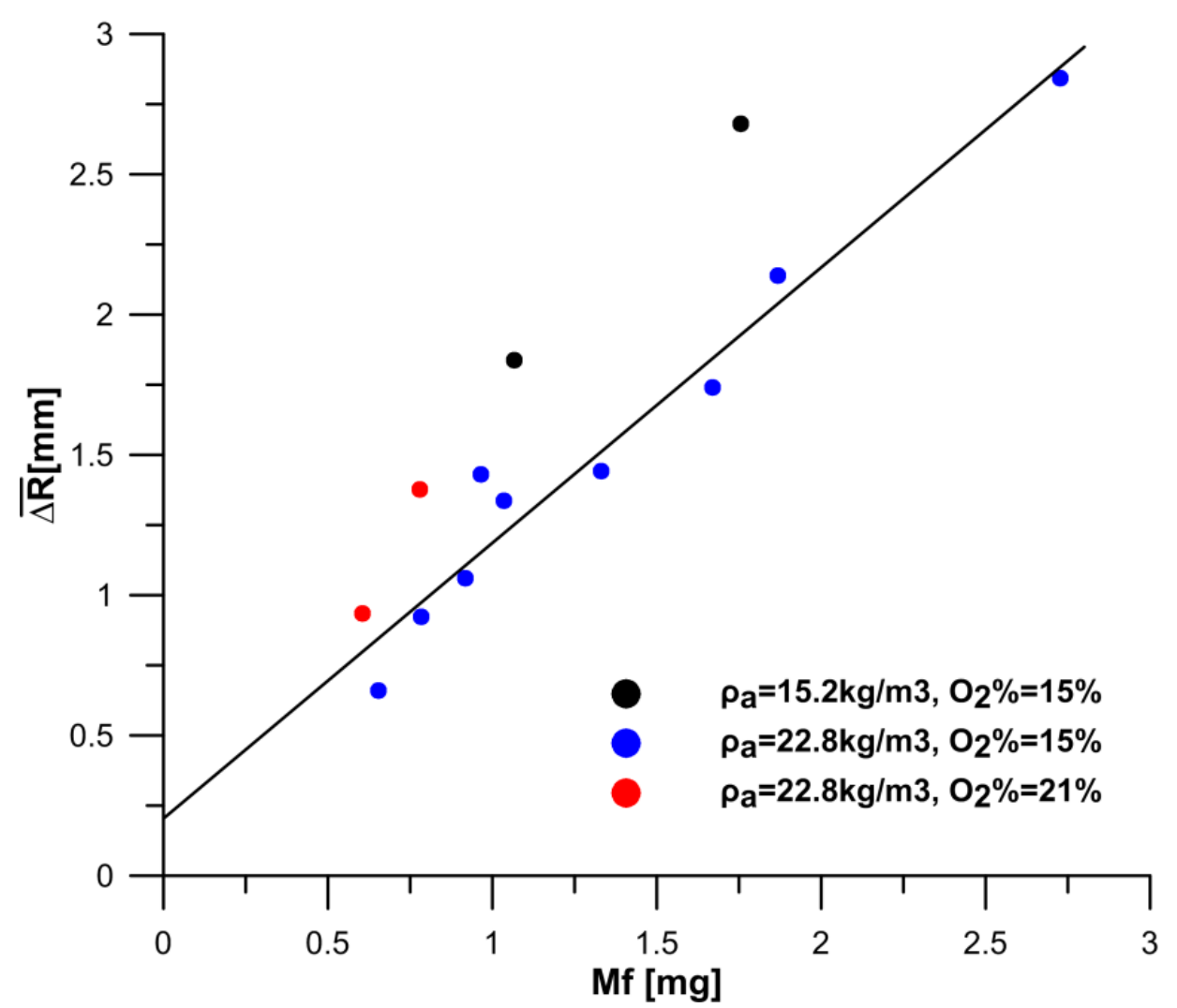

Fig.13. $\overline{\Delta \mathrm{R}}$ as a function of fuel mass from lift-off length to tip penetration at the start of combustion.

A clear linear relationship is observed in Fig. 13 between $\overline{\Delta R}$ and the fuel mass for the variation both injection pressure and ambient temperature. In other words, the difference of the expansion process caused by these two variables is governed by the amount of fuel mass in the igniting region (defined from the LOL until spray tip penetration). Injection pressure affects this characteristic fuel mass only in terms of the injection rate, as it has almost no effect on ignition delay $\left(P_{r}=1,500 \mathrm{bar}\right.$, $I D=0.46 \mathrm{~ms} ; P_{i}=1000 \mathrm{bar}, I D=0.49 \mathrm{~ms} ; P_{i}=1500 \mathrm{bar}, I D=0.52 \mathrm{~ms}$ ), while ambient gas temperature affects this characteristic fuel mass by the different ignition delay, with lower temperature leading to a longer ignition delay and therefore a higher injected mass. It must also be considered that both injection pressure and ambient temperature have a very limited effect on the spatial distribution of the equivalence ratio under inert conditions.

On the other hand, Fig. 13 shows that results for different ambient gas density and oxygen seem to follow a different although also linear trend. Both variables have a strong effect on the local equivalence ratio within the flame, either by modifying the mixture fraction distribution (density) or the oxygen content (oxygen). For a very similar fuel mass at the start of combustion, the equivalence ratio will certainly be different when changing any of both variables, compared to parametric variations of injection pressure and ambient temperature, which have a very limited effect on local equivalence ratio. As a result, resulting combustion temperatures and radial expansion effects will be different between oxygen/density parametric studies, and injection pressure/temperature ones.

The present analysis is only a first estimation of global effects during spray autoignition. More work is needed with detailed CFD simulations that can confirm the previous reasoning. 


\section{Conclusions}

In this study, Schlieren imaging technique has been performed to investigate the dynamics of the evolution of reacting sprays. All measurements were performed in a constant pressure flow vessel under a wide range of operating conditions. Spray penetration and radial width of the spray were investigated with particular focus to the axial and radial expansion of the spray occurring during the combustion. The most relevant observations and conclusions of this study are summarized below:

1) The temporal evolution of tip penetration, consistently with a previous work [12], can be divided into five phases: i) non-reacting, ii) auto-ignition expansion iii) stabilization, iv) acceleration and v) quasi-steady.

2) The radial width was calculated from the probability contours. From the analysis of the results it emerged that the shape of the reacting spray can be divided into three regions:

I. Quasi-steady inert: from nozzle tip to the Lift-off length.

II. Quasi-steady reacting: from lift-off length to the position where the contour stops increasing radially.

III. Transient: from the end of the previous zone to the spray tip.

3) After comparing of the radial width of reacting and inert spray at the same tip penetration, it was found that the contour at the quasi-steady reacting part is almost parallel to the corresponding part in the inert case. In other words, the radial contour of the reacting spray is simply the result of shifting the inert one by an approximately constant value.

4) After the analysis of axial and radial expansions under different test conditions the authors observed a relationship between these quantities and the amount of fuel burned during at the onset of combustion. As a consequence of that the following experimental observations have been explained as below:

a. The radial expansion increases with higher injection pressure and lower ambient gas temperature. It was found with constant ambient density and oxygen concentration, the time-averaged radial width difference increases linearly with fuel mas downstream the lift-off length at the onset of combustion. But higher injection pressure increases fuel mass before combustion by means of a higher mass flow rate, while lower temperature increases it by longer ignition delay.

b. The higher ambient gas density increases gas entrainment. However, this causes a reduction in the initial combustion portion because of the shorter ignition delay and lift-off length. As a consequence of that, the radial expansion decreases with higher ambient gas density.

c. Oxygen concentration has no significant effect on the radial expansion. Although the higher oxygen ratio can lead to a leaner equivalence ratio for combustion, it is compensated by the shorter ignition delay which reduces the fuel mass during the ignition delay. 


\section{Acknowledgements}

Authors acknowledge that some equipment used in this work has been partially supported by FEDER project funds (FEDER - ICTS - 2012 - 06), framed in the operational program of unique scientific and technical infrastructure of the Ministry of Science and Innovation of Spain.

\section{References.}

[1] R. Kiplimo, E. Tomita, N. Kawahara, and S. Yokobe, Effects of spray impingement, injection parameters, and EGR on the combustion and emission characteristics of a PCCI Diesel engine, Applied Thermal Engineering 37 (2012) 165-175.

[2] R. Mobasheri, Z. Peng, and S. M. Mirsalim, Analysis the effect of advanced injection strategies on engine performance and pollutant emissions in a heavy duty DI-Diesel engine by CFD modeling, International Journal of Heat and Fluid Flow 33 (2012) 59-69

[3] Haifeng Liu, Zhaolei Zheng, Mingfa Yao and Peng Zhang, Influence of temperature and mixture stratification on $\mathrm{HCCl}$ combustion using chemiluminescence images and CFD analysis, Applied Thermal Engineering, 33-34(2012) 135-143

[4] Jesús Benajes, Santiago Molina, Antonio García, Eduardo Belarte, Michel Vanvolsem, An investigation on $\mathrm{RCCl}$ combustión in a heavy duty diésel engine using in-cylinder blending of diésel and gasoline fuels, Applied Thermal Engineering, 63(2014) 66-76

[5] J. D. Naber, Dennis L. Siebers, Effects of Gas Density and Vaporization on Penetration and Dispersion of Diesel Sprays, SAE Technical Paper, 960034 (1996)

[6] I. V. Roisman, L. Araneo, C. Tropea, Effect of ambient pressure on penetration of a Diesel spray. International journal of multiphase flow, 33:8 (2007) 904-920

[7] F. Payri, R. Payri, M. Bardi, M. Carreres, Engine combustion network: Influence of the gas properties on the spray penetration and spreading angle. Experimental Thermal and Fluid Science, 53 (2014) 236-243

[8] M. P. B. Musculus, K. Kattke, Entrainment waves in Diesel jets, SAE Technical Paper, 2009-01$1355(2009)$

[9] John E. Dec, A Conceptual Model of DI Diesel Combustion Based on Laser-Sheet Imaging, SAE Technical Paper, 970873 (1997)

[10]M. P. B. Musculus, P.C. Miles, L. M. Pickett, Conceptual models for partially premixed lowtemperature Diesel combustion, Progress in Energy and Combustion Science, 39.2 (2013) 246283

[11]D., Siebers, Chapter 5 in Flow and combustion in reciprocating engines. Springer, 2009

[12] Pickett, L.M., Hoogterp, L. Fundamental spray and combustion measurements of JP-8 at diesel conditions (2009) SAE International Journal of Commercial Vehicles, 1 (1), pp. 108-118.

[13] J. M. Desantes, J. V. Pastor, J. M. García-Oliver, F. J. Briceño, An experimental analysis on the evolution of the transient tip penetration in reacting Diesel sprays, Combustion and Flame, 161:8 (2014) 2137-2150

[14]Pickett, L.M., Kook, S., Williams, T.C. Visualization of diesel spray penetration, cool-flame, ignition, high-temperature combustion, and soot formation using high-speed imaging(2009) SAE International Journal of Engines, 2 (1), pp. 439-459 
[15] Engine Combustion Network webpage: http://www.sandia.gov/ecn (online). Last accessed December 2014

[16]M. Bardi, R. Payri, L. M. Malbec, G. Bruneaux, L. M. Pickett, J. Manin, T. Bazyn, C. Genzale, Engine combustion network: comparison of spray development, vaporization, and combustion in different combustion vessels, Atomization and Sprays, 22:10 (2012) 807-842

[17]R. Payri, J. M. García-Oliver, M. Bardi, J. Manin, Fuel temperature influence on Diesel sprays in inert and reacting conditions, Applied Thermal Engineering 35 (2012) 185-195

[18] M. Meijer, B. Somers, J. Johnson, J. Naber, S.-Y. Lee, L. M. Malbec, G. Bruneaux, L. M. Pickett, M. Bardi, R. Payri, T. Bazyn, Engine Combustion Network (ECN): Characterization and comparison of boundary conditions for different combustion vessels, Atomization and Sprays 22 (2012) 777-806

[19]J. Benajes, R. Payri, M. Bardi, P. Martí-Aldaraví, Experimental characterization of Diesel ignition and lift-off length using a single-hole ECN injector, Applied thermal engineering 58 (2013) 554563

[20] G. S. Settles, Schlieren and sadowgraph techniques: visualizing phenomena in transparent media (Experimental Fluid Mechanic), Springer Verlag, Heidelberg, Germany, 2001

[21] H. Kosaka, T. Aizawa , T. Kamimoto ,Two dimensional imaging of ignition and soot formation processes in a Diesel flame, International Journal of Engine Research, 6:1 (2005) 21-42

[22] L. M. Pickett, J. Manin, C. L.Genzale, D. L. Siebers, M. P. B. Musculus, C. A. Idicheria, Relationship between Diesel fuel spray vapor penetration/dispersion and local fuel mixture fraction, SAE Technical paper 2011-01-0686, (2011)

[23] J.V. Pastor, J.J. López, J.M. García, J.M. Pastor, A 1D Model for the Description of MixingControlled Inert Diesel Sprays, Fuel, 87 (2008) 2871-2885

[24]J.V. Pastor, J.J. López, J.M. García, J.M. Pastor, A 1D Model for the Description of MixingControlled Reacting Diesel Sprays, Combustion and Flame 156 (2009) 234-249 


\title{
A study on Diesel spray tip penetration and radial expansion under reacting conditions
}

\author{
Raul. Payri, Jose.M. García-Oliver ( $\left.{ }^{\star}\right)$, Tiemin. Xuan \\ CMT Motores Térmicos - Universitat Politècnica de València, Valencia (Spain) \\ Michele. Bardi \\ LAV - Swiss Federal Institute of Technology, Zurich (Switzerland) \\ (*) Corresponding Author. Contact data: email: igarciao@mot.upv.es Tel: +34 963877650 \\ Camino de Vera s/n 46022 Valencia - Spain
}

\begin{abstract}
The shape of Diesel spray was investigated at real engine conditions in a constant pressure combustion chamber. Schlieren imaging technique was used to make quantitative measurements of spray tip penetration and radial width stressing the impact that the fuel combustion and heat release have on the spray shape. The heat-release region and the Lift-off length were identified measuring $\mathrm{OH}^{*}$ chemiluminescence. The fuel (n-dodecane) as well as the operating conditions and the injector used (single axially-oriented hole, $89 \mu \mathrm{m}$-diameter) were chosen following the guidelines of the Engine Combustion Network. The effects of different operating parameters on the axial and radial expansion were also investigated. According to the results the reacting spray can be divided into three parts: an inert part, a transient one, and a quasi-steady one that lays between the two other regions. A new method for evaluating this radial expansion of reacting spray was developed, which was evaluated under the different operating conditions. Results show that the radial expansion increases with increasing injection pressure and decreasing ambient temperature and ambient density. The oxygen concentration has no obvious effect on the radial expansion.
\end{abstract}

Keywords: tip penetration; radial expansion; reacting spray; Schlieren imaging

\section{Highlights:}

Spatial evolution of the reacting spray can be divided into three parts.

The radial contour of the reacting spray is the result of shifting the inert one by an approximately constant value

The radial expansion increases with higher injection pressure and lower ambient gas temperature.

The radius expansion decreases with higher ambient gas density.

Oxygen concentration has no significant effect on the radial expansion.

Nomenclature

ASOI After the start of injection ( $\mu \mathrm{s})$

CFD Computational Fluid Dynamics (-)

CPF Constant-pressure flow facility 
ECN Engine Combustion Network (-)

ICCD Intensified CCD sensor (-)

$\mathrm{k}_{\mathrm{r}}$ the slope of fitting line

LOL lift-off Length ( $\mathrm{mm}$ )

$\mathrm{O}_{2} \%$ Oxygen concentration (-)

$\mathrm{P}_{\mathrm{i}} \quad$ Injection pressure (bar)

$\overline{\Delta \mathrm{R}}$ the time-averaged radial difference of the quasi-steady part between reacting and inert spray contour $(\mathrm{mm})$

SOC Start of combutstion $(\mu \mathrm{s})$

$\mathrm{S}_{\mathrm{i}}$ Inert spray tip penetration $(\mathrm{mm})$

$\mathrm{S}_{\mathrm{r}}$ Reacting spray tip penetration $(\mathrm{mm})$

$\mathrm{T}_{\mathrm{a}}$ Ambient gas temperature $(\mathrm{K})$

ID Ignition delay $(\mu s)$

Greek symbols

$\mathrm{Pa}$ gas density $\left(\mathrm{kg} / \mathrm{m}^{3}\right)$

$\rho_{\mathrm{f}}$ fuel density $\left(\mathrm{kg} / \mathrm{m}^{3}\right)$

$\theta_{\mathrm{r}}$ Spreading angle of reacting spray

\section{Introduction}

Fuel-air mixing plays a significant role in combustion and emission process in diesel engine, which can be controlled by different injection parameters [1], injection strategies [2], advanced combustion modes[3] or fuel blending [4]. The complexity of the phenomena characterizing this process brought a significant challenge to the research community boosting the investigations in this field: experimental and computational tools have been significantly developed along the last decade and important goals have been achieved.

The definition and experimental characterization of many parameters related to specific spray features (tip penetration, spreading angle, liquid phase penetration, lift-off length etc) brought an important step forward in the understanding of the process [5][6][7]. Thanks to that, conceptual and numerical models have been developed to reproduce the whole Diesel injection process outlining the complex physical and chemical processes that occur behind the experimental observations [1][8].

Probably the most significant contribution in this direction are two conceptual models for conventional combustion and low temperature combustion based on different optical techniques proposed in [9] and [10], which give detail elaboration about how fuel-air mix in reacting conditions.

The increasing need for more accurate and predictive models together with the possibility of performing more detailed experiments still highlight some lack of knowledge. Few works in the literature address the effect that combustion has on the shape and mixing of the spray. Siebers's study [11] shows that the reacting spray evolution depends on the ambient density. The similar trend was also observed by Pickett and Hoogterp [12]. The spray penetration under reacting conditions was 
studied by Desantes et al. [13] and one defined spreading angle was applied for representing the radius expansion. In reference [14], the reacting and non-reacting spray penetration was also investigated with schileren technique. In most of such works, analysis is mainly based upon the effect of heat release on spray tip penetration by comparing the spray behavior when injected into an inert $(\mathrm{O} 2=0 \%)$ or a reacting $(\mathrm{O} 2>0 \%)$ atmosphere. Following a similar approach, the main goals of the present study are 1) to highlight the modification in the spray shape observed at reacting conditions 2) to investigate the mechanism behind these observations and 3) to provide quantitative data to the modelers that approach this problem.

This work is framed within the activities of the Engine Combustion Network (ECN): the ECN is a worldwide group of research institutes that started a collaboration to provide high quality data and consistent results to modelers [15]. The work of the group lays on several coordinated efforts in the Diesel research field: the complete definition of a standard reference condition called Spray A condition (and defining suggested parametric variations of the boundary conditions), the use of nominal identical injectors and the organic cross check of the data obtained by different facilities and through different techniques [16]. In this way, the results provided in this paper will also contribute to extend and integrate the open-access database provided by the community.

In the present work, the experiments were performed in a constant-pressure flow facility (CPF) able to reach 150 bar ambient pressure and $1,000 \mathrm{~K}$ ambient temperature. The optical diagnostics performed are:

- Schlieren imaging, to detect complete boundary of the inert and reacting spray

- $\mathrm{OH}^{*}$ chemiluminescence to measure lift-off length and to highlight the high-temperature heat release region of the reacting spray

The test matrix has been defined to isolate the effect of the combustion on the spray development: a set of tests was performed injecting the fuel in high temperature high pressure atmosphere but in pure Nitrogen. Then the set of tests have been repeated at the same thermodynamic conditions but increasing the oxygen content ( $15 \%$ and $21 \%$ (vol.)).

The analysis of the results focuses on the understanding of the impact that the combustion has on the modification of the spray boundaries defining also a criterion to quantify what will be defined afterward as radial expansion of the spray.

Including the present introduction, this document is composed of four sections. The next section gives a detailed description about the experimental apparatus, the setup of the two optical techniques, the different operating conditions which were investigated in this paper and the image processing. In the third section, the results under inert and reacting conditions were analyzed and discussed. The last section of the paper summarizes some of the most important conclusions of this investigation. 


\section{Experimental setup and Methodology}

\subsection{Experimental facility}

A constant-pressure flow (CPF) test chamber, as shown in Fig. 1, capable of reproducing typical incylinder thermo-dynamic conditions of a Diesel engine was used. Three wide quartz windows (120 $\mathrm{mm}$ diameter) enable a favorable optical access to the test section. Compared to other facilities this test rig has the unique feature of obtaining nearly quiescent and steady thermodynamic conditions within the chamber, providing thus an important reduction in the time required for the tests and higher accuracy in the control of the boundary conditions [17]. A separated cooling system was used for the injector: the temperature of the coolant was adjusted in order to maintain constant the temperature of the nozzle tip in the different test conditions. The calibration of the injector temperature control system was presented in [17]. The thermodynamic conditions within the chamber have been deeply characterized in [18] measuring the temperature at different positions with thermocouples of different diameters and accounting for needed the radiation correction [18]. A complete description of the facility is given in [17].

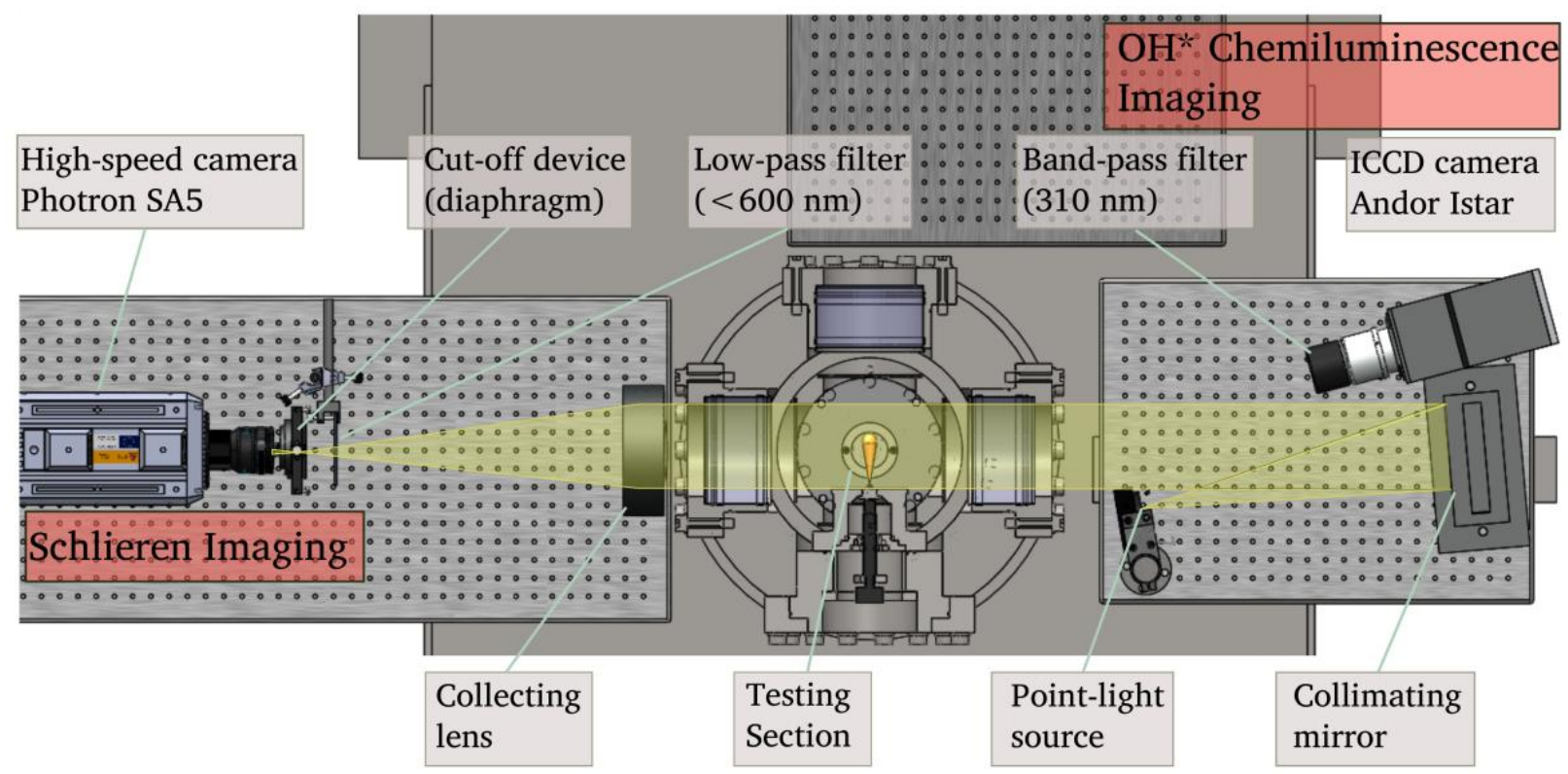

Fig. 1. Experimental layout: the test chamber is presented together with the optical setup.

\subsection{Test matrix}

The investigated operating conditions are summarized in Table 1 which is same as that in the reference [19] where the focus was on ignition and LOL, while here more details are given on the dynamics of the spray. The boundary conditions chosen aim at the reference Spray $A$ operating conditions ( $\left.T_{a}=900 \mathrm{~K}, \rho_{a}=22.8 \mathrm{~kg} / \mathrm{m}^{3}, P_{i}=1,500 \mathrm{bar}\right)$ and the parametric variation of the main variables as defined by ECN Working Group [16]. As previously mentioned an objective of the tests is the comparison of the spray contours measured at inert and reacting conditions. The parametric variation in the injection parameters (injection pressure) and thermodynamic conditions within the 
chamber (ambient temperature and ambient density) were performed by injecting the fuel into pure nitrogen (inert condition) or into an atmosphere with nitrogen and different percentages of oxygen (15, $21 \%$ (vol.)). In order to improve the statistical reliability of the results presented, 8 injection cycles were recorded for each operating condition.

Table 1. Operating conditions

\begin{tabular}{lll}
\hline Parameters & values & units \\
\hline Fuel & $\mathrm{n}$-Dodecane & - \\
Nozzle diameter & 89 & $\mu \mathrm{m}$ \\
Nozzle k-factor & 2.1 & - \\
Energizing time & 3500 & $\mu \mathrm{s}$ \\
Injection duration & 5200 & $\mu \mathrm{s}$ \\
Tip temperature & 390 & $\mathrm{~K}$ \\
Injection pressure $\left(\mathrm{P}_{\mathrm{i}}\right)$ & $500 ; 1,000 ; 1500$ & $\mathrm{bar}$ \\
Ambient gas temperature $\left(\mathrm{T}_{\mathrm{a}}\right)$ & $800 ; 850 ; 900$ & $\mathrm{~K}$ \\
Ambient density $\left(\rho_{\mathrm{a}}\right)$ & $15.2 ; 22.8$ & $\mathrm{~kg} / \mathrm{m}^{3}$ \\
Oxygen concentration $\left(\mathrm{O}_{2} \%\right)$ & $0 ; 15 ; 21$ & $\%(\mathrm{vol})$. \\
\hline
\end{tabular}

\subsection{Injection system}

The injection system employed consists of commercial available components: a high pressure volumetric Bosch CP3 pump driven by an electric motor; a common rail with pressure regulator controlled by a PID system.

The injector employed, based on second generation Common-Rail, is part of the ECN injector dataset (ref: \#210675 [15]). The injector features a single-hole axial nozzle enabling the advantage to focus on the fundamental behavior of the spray eliminating both the effects and the uncertainties related to the temperature boundary layer close to the walls and the spray-spray interaction. Moreover, ECN the internal geometry of the nozzle and the corresponding hydraulic behavior has been deeply characterized and the data are available online [15].

Following the ECN guidelines, a single-component fuel has been employed ( $n$-dodecane) to facilitate the comparison with CFD simulations.

\subsection{Optical techniques and processing methods}

Two imaging techniques were employed simultaneously in this study:

- High-speed Schlieren

- Time averaged $\mathrm{OH}^{*}$ chemiluminescence

The details of the measurements and of the setup employed are given in the following sections and the sketch of the experimental layout is presented in Fig. 1.

Table 2. Details of the optical setup used in the study.

\begin{tabular}{lcc}
\hline & $\begin{array}{c}\text { Schlieren } \\
\text { Imaging }\end{array}$ & $\mathrm{OH}^{*}$ chemilum. \\
\hline Camera & Photron SA-5 & Andor - Istar
\end{tabular}




\begin{tabular}{lcc} 
Sensor Type & CMOS & ICCD \\
Lens & $50 \mathrm{~mm}$ & $100 \mathrm{~mm}-$ U.V. \\
Diaphragm & $4 \mathrm{~mm}$ & - \\
Filter & - & $310 \mathrm{cwl} \pm 5$ \\
Frame Rate & $50 \mathrm{kfps}$ & 1 frame/injection \\
Shutter time & $4 \mu \mathrm{s}$ & $3 \mathrm{~ms}$ (from 2 to $5 \mathrm{~ms}$ ASOI) \\
Repetitions & 8 & 15 \\
Pixmm & 5.26 & 5.85 \\
\hline
\end{tabular}

\subsubsection{High speed Schlieren imaging}

A conventional Schlieren single-pass arrangement was employed to detect the complete spray boundaries at high temperature and high pressure conditions.

Schlieren imaging technique enables to detect gradients in the refractive index of a transparent medium [19]. The technique relies on the deviation of a light beam produced when light passes through non-homogeneous fluids. A Diesel spray injected at high temperature and high pressure conditions causes variations of refractive index gradient for three main reasons: a) the different optical properties between the fuel and the ambient gas b) the gradients in density /temperature that are generated within the spray related to the mixing process $\mathrm{c}$ ) the heat released during the combustion.

As a consequence, images acquired show the spray boundaries including liquid and vapor phase fuel, and, under reacting conditions, the regions which contain combustion products.

The Schlieren setup employed (Fig. 1) includes different components: Xe-Arc lamp and a $1 \mathrm{~mm}$ diameter pin-hole constituting the point light source of the system; a parabolic mirror used to collimate the light from the pin-hole in a parallel beam; a collecting lens to focus the parallel beam in a single point and a high-speed camera(50 kfps). A diaphragm (4 mm aperture) was used as a cut-off device to enhance the sensitivity of the setup while a short-pass filter $(<600 \mathrm{~nm})$ was filtering the light beam to reject part of the soot incandescence light emissions. More details of the setup are indicated in Table 2.

The images captured in the tests were processed following an intensity-based criterion and applying a dynamic background subtraction. The methodology, described in details in the work by Benajes et al. [19], proved to be a robust one and provides binarized images of the spray region.

From each single spray image a binarization process results in a corresponding contour (Fig. 2) and a spray tip penetration. Following this processing step, a statistical analysis makes it possible to derive the following parameters:

- Average spray tip penetration (s) by averaging of the corresponding sample of repetitions for each time step.

- An average spray boundary for a given time step, which is obtained by calculation of spray probability maps from the corresponding sample of binarized images (Fig. 2). Such maps indicate the probability of finding a spray at one position. The average spray boundary is defined as the border of the region where the probability is equal to or higher than $50 \%$. Moreover, considering the spray axisymmetric, the spray contour was summarized in only two variables: axial position, and spray width. 
Schlieren images

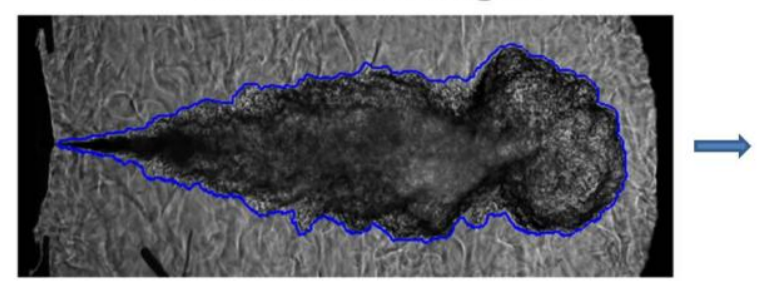

Binarization

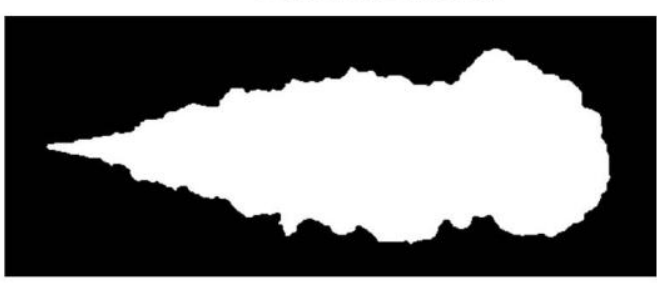

Rep.

\#1

\#8

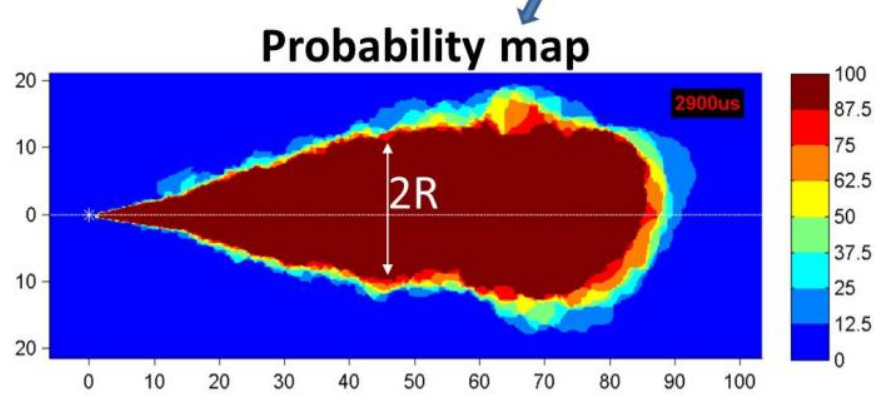

Fig. 2. The image processing workflow for Schlieren-derived images

\subsubsection{Time averaged $\mathrm{OH}^{*}$ chemiluminescence}

The high-temperature heat release region of a Diesel spray is generally related with the light emissions by the $\mathrm{OH}^{*}$ radicals decaying at their ground state. These light emissions have an important peak at $309 \mathrm{~nm}$ [19]. An ICCD camera (Andor I-star) fitted with a $100 \mathrm{~mm} \mathrm{f} / 2.8 \mathrm{UV}$ lens and a $310 \pm 5 \mathrm{~nm}$ interferometric filter was used with a constant intensifier gating time widow synchronized with the injection between 2.0 and $5.0 \mathrm{~ms}$ after the start of the injections (ASOI): in this way the steady part of the combustion process was averaged along the injection event and the shot-to-shot deviation was reduced. The $\mathrm{OH}^{*}$ chemiluminescence images were employed for the calculation of the Lift-off length. The procedure followed and the results have been already presented in [19].

\section{Results and discussion}

\subsection{Reference analysis at Spray A conditions}

Taking into account the limitations of the optical access of the CPF chamber and the frequency of the cases used for comparison in later sections, the baseline Spray A condition was selected for the reference analysis in this section. Because of the early ignition caused by high ambient temperature and high density and fast spray development created by high injection velocity, the full development period of the spray can be recorded by the camera before the end of injection.

Fig. 3 and Fig. 4 (right) show the temporal evolution of the tip penetration and radial width of inert and reacting sprays under Spray A condition, respectively. In these figures as well as in the subsequent ones presented in this paper, the time is referred to the start of the injection with the acronym ASOI (After Start Of Injection). Sample images from a single injection cycle are shown in Fig. 4 (left). As shown in top plot of Fig.3, the red and the blue solid line represent the average spray penetration from the eight repetitions under reacting and inert conditions, respectively. Due to the very 
small difference in molecular weight between oxygen and nitrogen, ambient pressure is essentially the same for the nominal ambient density and temperature values. The vertical red dashed line in Fig. 3 represents the start of combustion obtained from Schlieren images following the methodology in [20]. Both inert and reacting spray tip penetrations increase steadily with time, but the reacting case shows an apparent acceleration after the start of combustion. To further analyze this behavior, the bottom plot shows the penetration ratio (i.e. reacting divided by inert penetration at each time step), consistently with the analysis carried out in a previous work from Desantes et al [13] for n-heptane sprays under similar operating conditions. As described in that paper, the temporal evolution of the reacting spray can be divided into five stages:

I.Non-reacting phase: from the start of injection to the start of combustion, where the penetration ratio should be essentially one. Results in Fig.3 shows values lower than one. This is due to the small penetration values, so that scattering between inert and reacting tests may result in a relatively important deviations of the ratio from one. .

II.Auto-ignition expansion phase: It is a relatively short phase marked by a first short-lived peak in penetration ratio. It spans from the start of combustion (SOC) to the time when the penetration between inert and reacting condition become the same again.

III.Stabilization phase: from the end of the previous stage to the time when the tip penetration starts to separate, as shown in [13] the duration of this phase depends a lot on the operating condition, under less reacting conditions this phase becomes longer. Under Spray A conditions this phase is almost non-existent.

IV.Acceleration phase: where the penetration ratio starts increasing with time.

V.Quasi-steady phase: where the tip penetration ratio reaches a relatively steady value.

There is a decrease of the penetration ratio after the time exceeds $4 \mathrm{~ms}$ because the reacting case reaches the limit of the optical access, as shown in the top plot in Fig.3. 


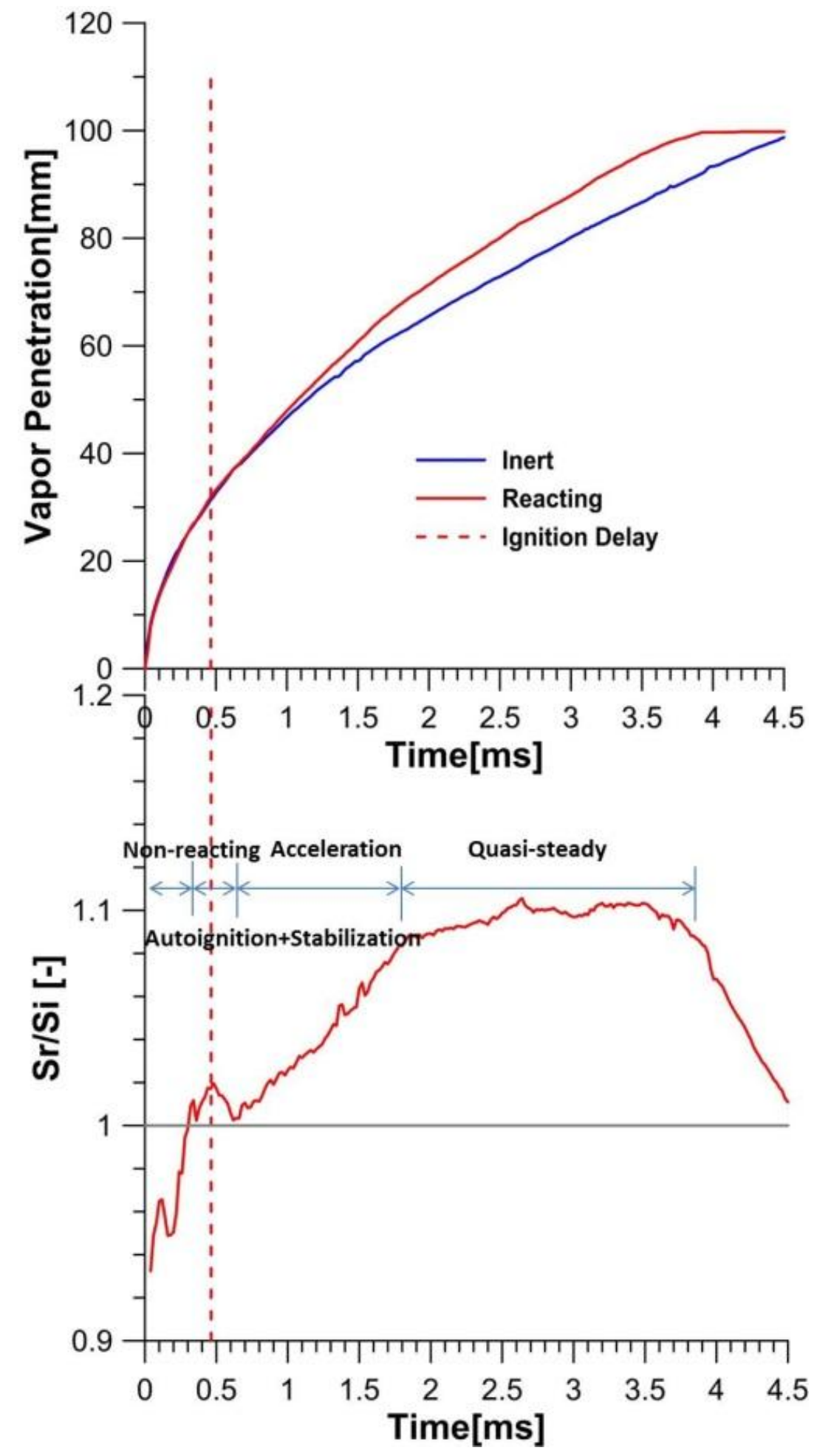

Fig. 3. Temporal evolution of the penetration and ignition delay (dashed line) from Schlieren image processing for both inert and reacting conditions (top) and corresponding penetration ratio(bottom). (Spray A condition)

The image sequence on the left hand side of Fig. 4 presents the temporal evolution of half images of a single injection for the reacting nominal Spray A condition. As mentioned above, the optical access was limited to $100 \mathrm{~mm}$ and the black area on the right top of these images is the window limit. As for the right image sequence of Fig. 4, the temporal evolution of the spray half-width (i.e. the spray radius) as derived from the probability images under reacting and inert conditions are 
shown here for the indicated timings. Differences between left and right image sequences of Fig. 4, are due to the comparison of average contours to instantaneous realizations of the experiment. The vertical red dashed line represents the average lift-off length as obtained from $\mathrm{OH}^{*}$ chemiluminescence images.

At $320 \mu \mathrm{s}$ ASOI, the spray behaves as an inert spray which corresponds to the non-reacting phase of the penetration mentioned above and there is no expansion neither in axial nor in radial direction. The contours of inert and reacting conditions still keep similar at $400 \mu \mathrm{s} \mathrm{ASOI}$, even though some part of the spray tip starts getting transparent as a consequence of the onset of cool flames. At about $450 \mu \mathrm{s}$ ASOI, the ignition takes place which directly leads to a separation of the penetration and radial expansion in the front part of the spray. This agrees with the auto-ignition expansion phase observed in the penetration plot. At $660 \mu \mathrm{s}$ ASOI, there is an obvious radial expansion for the reacting spray, and the tip penetration of both conditions become similar again. According to the momentum conservation, the strong increase in radial width leads to a slower penetration for the. This image

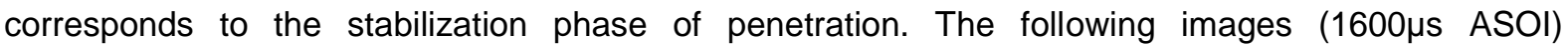
correspond to the acceleration phase of the penetration evolution. It can be seen that the initial radial expansion part downstream of the lift-off length is stabilized compared with the same part of the next plot $(2900 \mu \mathrm{s}$ ASOI), with a spray slightly wider than under inert conditions. At $1600 \mu \mathrm{s}$ ASOI a 'waist' part was found at around $60 \mathrm{~mm}$ from the nozzle where the reacting spray contour trends to be almost flat and overlaps with the inert spray contour. The last images (2,900us ASOI) correspond to the quasi-steady phase of the penetration. It was found that the stabilized part spans from the nozzle up to around $50 \mathrm{~mm}$. An apparent vortex came up after the jet head (left image), which can also be seen one the contour derived from the probability, which means that the 'waist' is statistically significative. This confirms that the transient tip of the spray is governed by a vortex with a very repetitive pattern. This phenomenon could also be found over a wide range of operating conditions which should be attributed to rapid gas entrainment [21]. 

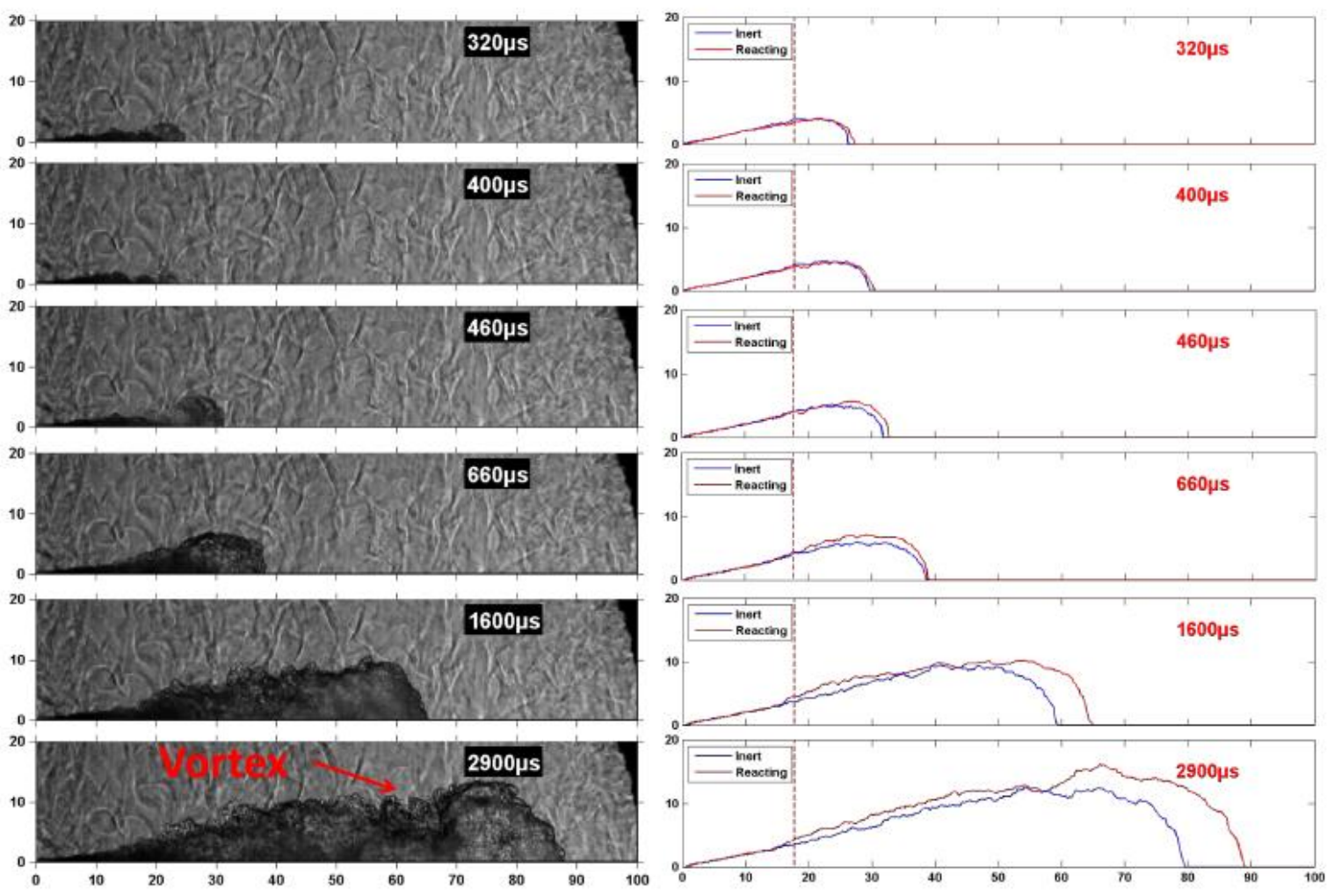

Fig. 4 Temporal evolution of the reacting spray contour obtained with Schlieren (left) and radial width both under inert and reacting condition(right) (spray A condition)

\subsection{Analysis of spatial evolution of reacting spray contour under baseline Spray A conditions}

Different approaches can be found in the literature to assess the radial dispersion of inert and reacting spray and many definitions for the spray spreading angle are given [5][13][22]. However, it was found that, especially at reacting conditions, the geometry of diesel spray is much more complicated than a cone shape and it was not well represented by a single parameter. In order to better understand the radial dispersion of the reacting spray, the radial widths for both inert and reacting spray under Spray A condition were compared. As shown in Fig.5, the red and blue solid lines represent the radius width of the reacting and inert spray separately. The red vertical dashed line represents the lift-off length as derived from $\mathrm{OH}^{*}$ chemiluminiscence images. It must be noted that the comparison of contours is performed at iso-spray tip penetration, not at the same timing. This means that while the time of inert spray is at $4380 \mu \mathrm{s}$ ASOI and the time of reacting spray is at $3620 \mu \mathrm{s}$ ASOI.

According to several experimental evidences, the structure of the reacting spray can be divided into 3 parts as shown in Fig. 3:

I.Quasi-steady Inert part, which is defined from the nozzle tip to the Lift-off length. Because of the absence of heat release, in this zone the spray behaves as an inert flow.

II.Quasi-steady reacting part, which is defined from lift-off length to the position where the contour stops increasing radially. In this part, the heat released by the oxidation reactions cause an increase in the radial width at every position of the spray axis. After a first 
transient where the radial width increases with the axial position, the difference remains almost constant throughout the region.

III.Transient part, which is defined from the end of the quasi-steady part to the spray tip. This part is much more complicated than other two ones, due to its transient nature. In the same way as for an inert spray, a reacting one is made up of a quasi-steady part (I and II) led by a transient tip. As sketched in Fig. 5, for a developed spray the extent of the steady part (I and II) is roughly $50 \%$ of the total spray tip penetration, compared to around $60 \%$ which is a reference value for the inert case, and agrees with the contour in Fig. 5.

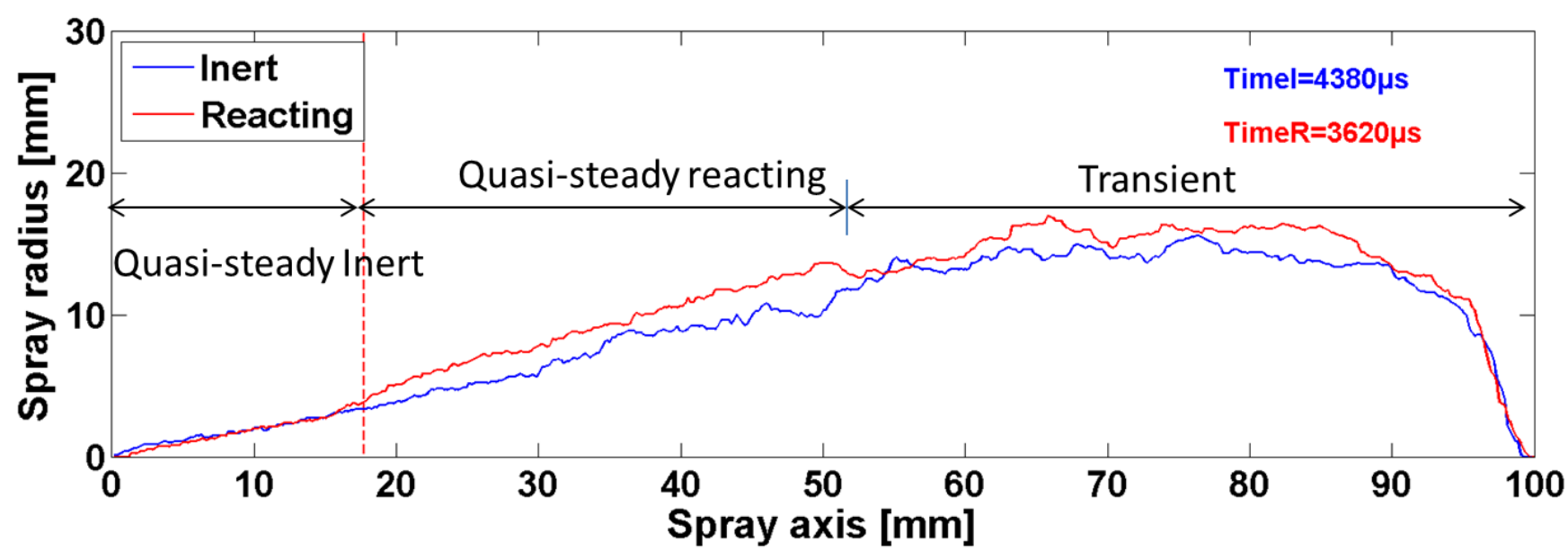

Fig. 5. Radial width for both inert and reacting spray under Spray $A$ condition $\left(S_{r}=97 \mathrm{~mm}, S_{i}=97 \mathrm{~mm}\right)$.

One of the aims of this paper is to gain understanding on the radial expansion characterizing the quasi-steady reacting region. To this end, it is necessary to define the position of the transition point from quasi-steady reacting part to the transient part. Two fitting lines to the inert and reacting spray contours were done by means of a least-squares algorithm from a distance to the nozzle $10 \%$ longer than the lift-off length to three different percentages $(40 \%, 50 \%, 60 \%)$ of the tip penetration. Fig. 6 shows the fitting lines with the contours of the three percentages at the same time. It is quite apparent that the $40 \%$ limit can be further extended downstream while still being valid, whereas the $60 \%$ case already runs into the transient tip. Accordingly, the lower limit of the transient tip will be defined hereafter as $50 \%$ of the tip penetration. 

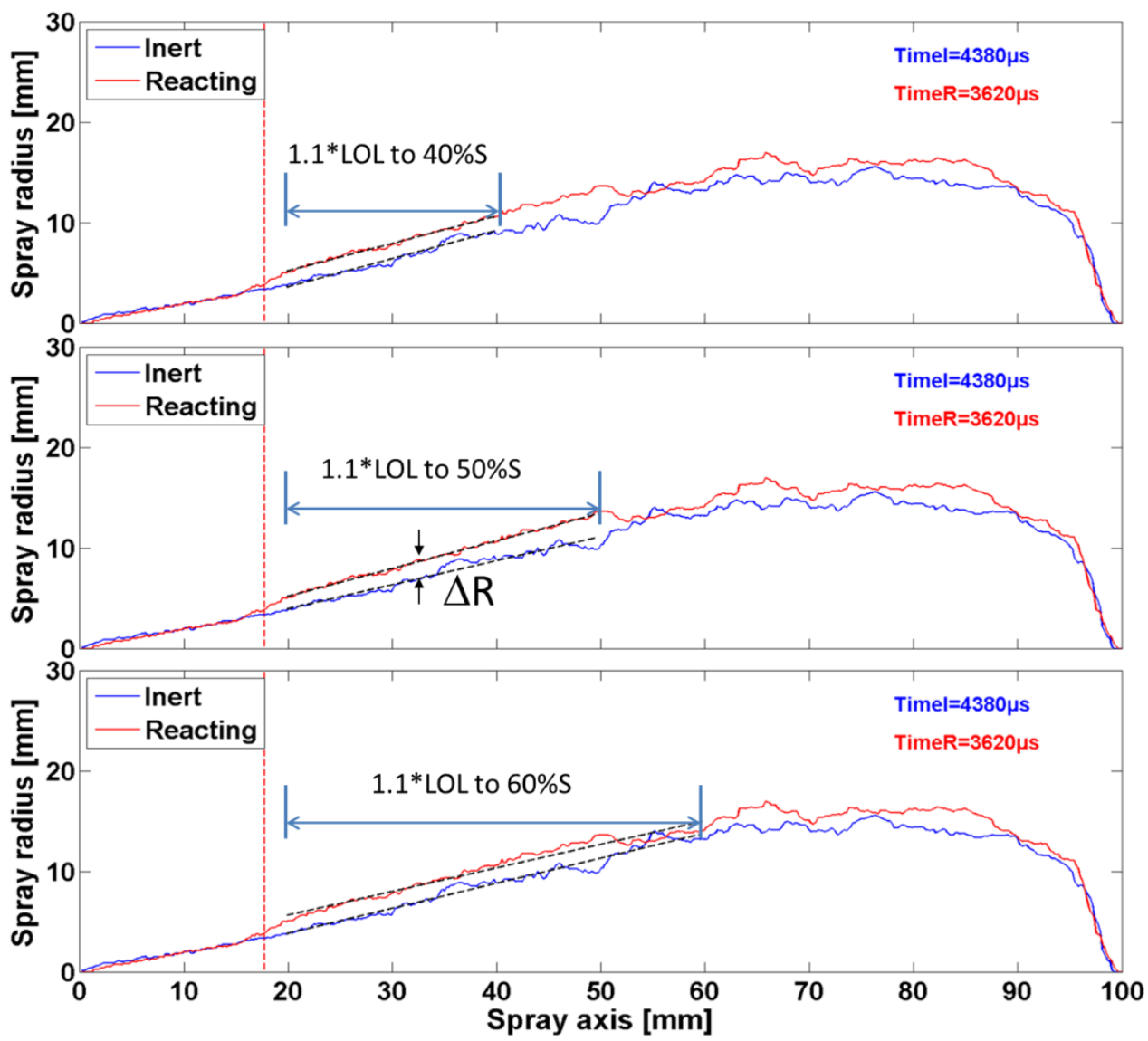

Fig.6. The fitting line of the inert and reacting spray at Spray A condition

From the derived contours, both inert and reacting spray cone angles were calculated according to the slope of the fitting line.

$$
\tan \left(\theta_{r} / 2\right)=k_{y}
$$

where $\theta_{r}$ is the spreading angle of the spray, $k_{r}$ is the slope of the fitting line.

The plots in Fig. 6 correspond to a single time instant. The corresponding time-resolved evolution of the spray angle under inert and reacting conditions is presented in Fig.6.

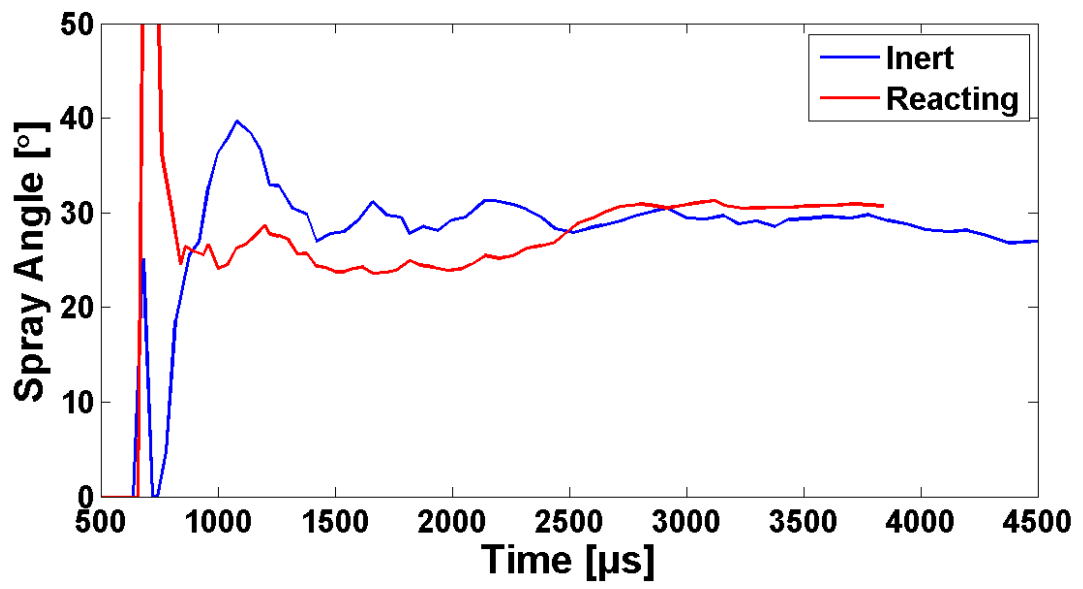

Fig. 7. The spread angle of the inert and reacting spray at Spray A condition 
Results show that both inert and reacting angles trend to be the same value after some time interval, i.e. the two fitting lines become parallel. This means that the radial expansion at different axis position of the quasi-steady reacting part is similar, in other words, the radial contour of the reacting spray is simply the result of shifting the inert one by an approximately constant value.

Based on the previous analysis, the radial increase should adequately describe the combustioninduced radial expansion along the whole spray axis. In order to find a single parameter to evaluate the radial expansion of the quasi-steady reacting part, the average value of the radial difference between inert and reacting conditions at every -time instant from a distance $10 \%$ longer than LOL to $50 \%$ of the spray tip has been calculated. After that, this spatially-averaged value was also timeaveraged into the parameter $\overline{\Delta \mathrm{R}}$. Because the reacting spray has not reached a stable states at the onset ignition period and the strong tip vortex has some influence on definition of this part, the time window for calculating the $\overline{\mathrm{AR}}$ was chosen from $1 \mathrm{~ms}$ to $2 \mathrm{~ms}$ after ignition delay. It has been observed that the defined parameter is stable in this time window for all cases except the low density case. One possibility for such a behavior is that the flame under low density condition is not as stable as that under high density. This quantitative indicator $\overline{\Delta \mathrm{R}}$ will be used in the following section to evaluate the expansion of the spray under parametric studies.

\subsection{Effect of operating parameters on reacting spray evolution}

\subsubsection{Effect of injection pressure and ambient gas temperature}

The temporal evolution of the effect of injection pressure on the penetration ratio and radius difference is shown in Fig. 8 The solid and dashed lines represent the penetration ratio and radius increase, respectively. The time window applied for calculating the time-averaged $\overline{\mathrm{AR}}$ was marked by the shadowed area in Fig.8 It can be observed that the penetration ratio at the quasi-steady phase increased with lower injection pressure This trend is not consistent with previous work [12], where the penetration ratio is shown to be similar during the final quasi-steady phase for different injection pressures, although n-heptane was used as a fuel, which is less reactive than n-dodecane. Regarding the radius width difference $(\Delta \mathrm{R})$, it keeps stable during the averaging time window and it eventually increases, which is probably caused by the strong vortex behind the jet head. The onset time of the quasi-steady phase of the penetration ratio is shown to have no relationship with the stable period of the radial difference. Comparing both penetration ratio and radius increase it can be concluded that the penetration ratio at the quasi-steady phase decreases with higher radial expansion. 


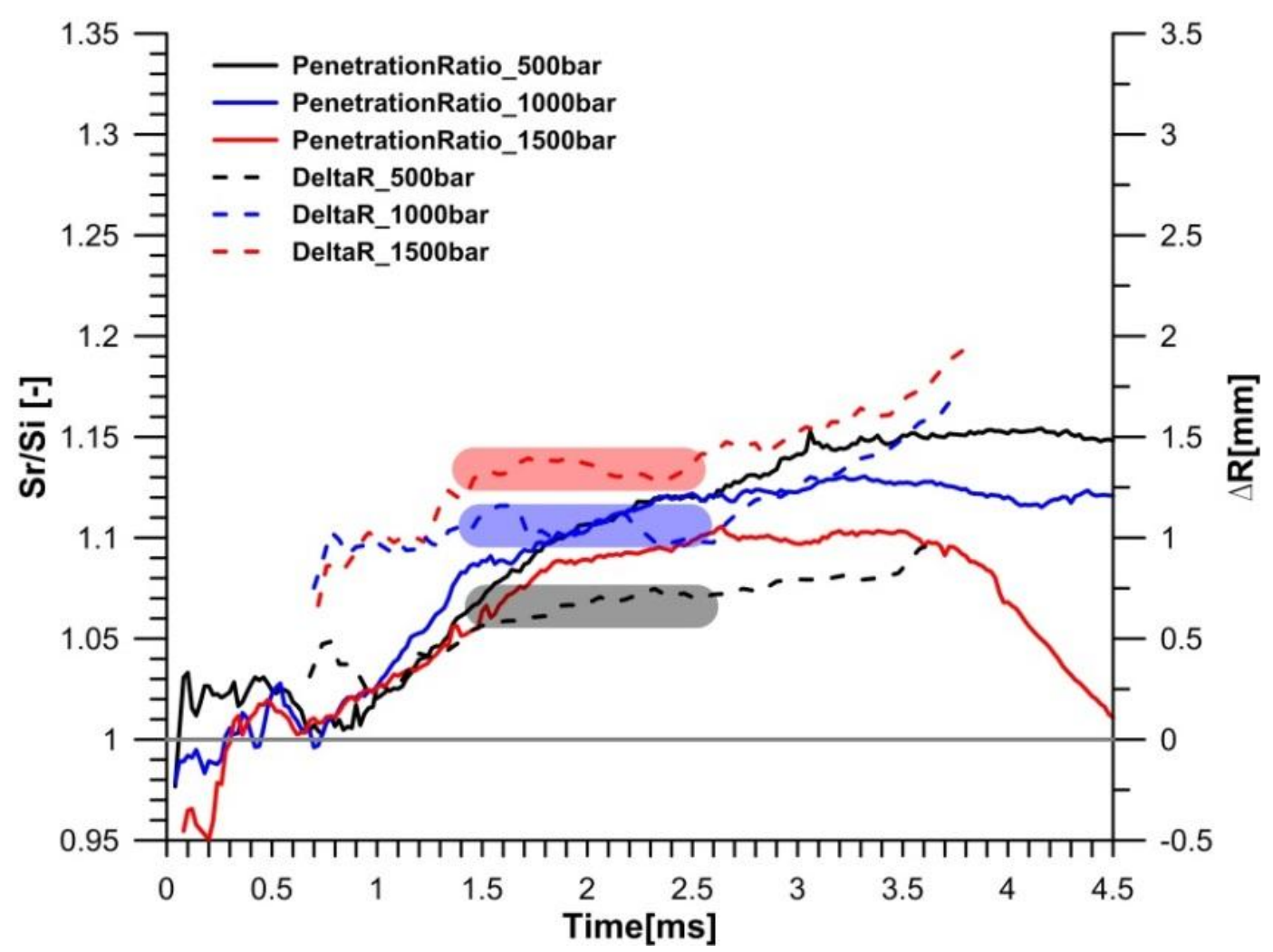

Fig.8. Temporal evolution of effects of injection pressure on penetration ratio and radius difference. $T_{a}=900 \mathrm{~K}, \rho_{a}=22.8 \mathrm{~kg} / \mathrm{m}^{3}, O_{2} \%=15 \%$.

On the other hand, Fig.9 shows that the temperature has a large effect both on the penetration ratio and radial expansion. With decreasing ambient temperature, the spray has a later ignition and a longer $\mathrm{LOL}$ obtained from $\mathrm{OH}^{*}$ chemiluminescence resulting in a leaner combustion. In terms of the spray penetration ratio, a longer duration of the stabilization phase is observed at lower ambient temperature, and most of the time along this phase the reacting spray tip penetrates slower than the inert one. As for the acceleration phase, the increasing rate and the peak value of the penetration ratio are similar for $850 \mathrm{~K}$ and $900 \mathrm{~K}$ temperature cases, while at $800 \mathrm{~K}$ both are lower.

As for the radial expansion, the value during the stable stage shows an increase with lower ambient temperature. Except the effect of vortex, a second factor, contributing to an increase on the radial difference at the later period of the evolution is the ending of the injection energizing after $3.5 \mathrm{~ms}$, although it is probably less significant. 


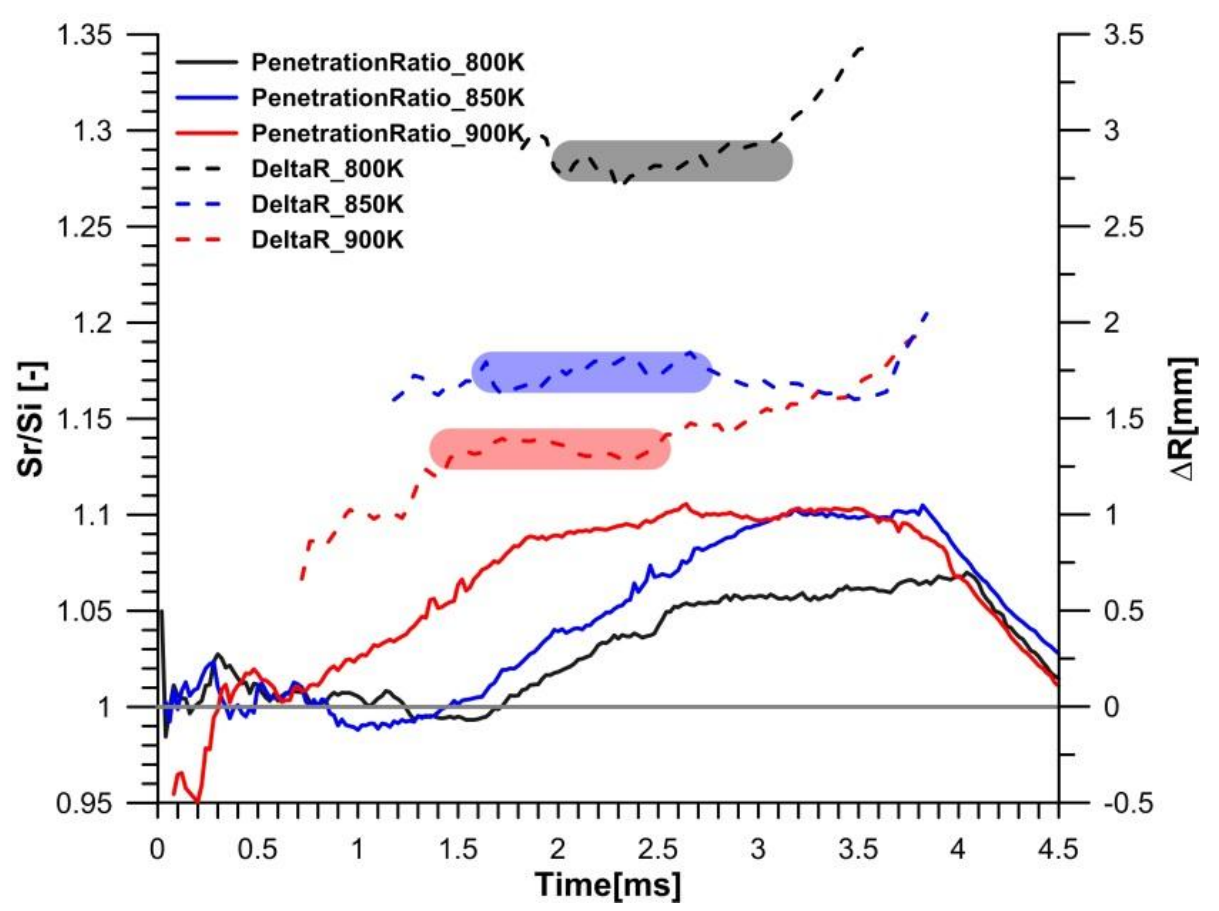

Fig.9. Temporal evolution of effects of ambient temperature on penetration ratio and radius difference. $P_{i=1,500 \mathrm{bar},} \rho_{a}=22.8 \mathrm{~kg} / \mathrm{m}^{3}, O_{2} \%=15 \%$.

The influence of injection pressure on the time-averaged $\overline{\Delta \mathrm{R}}$ at the three different temperatures is shown in Fig.10 The $\overline{\Delta \mathrm{R}}$ shows an increasing trend with higher injection pressure, the sensitivity is reduced with higher temperature. On the other hand, the plot also indicates that the temperature has a strong effect on $\overline{\Delta \mathrm{R}}$, especially under higher injection pressure.

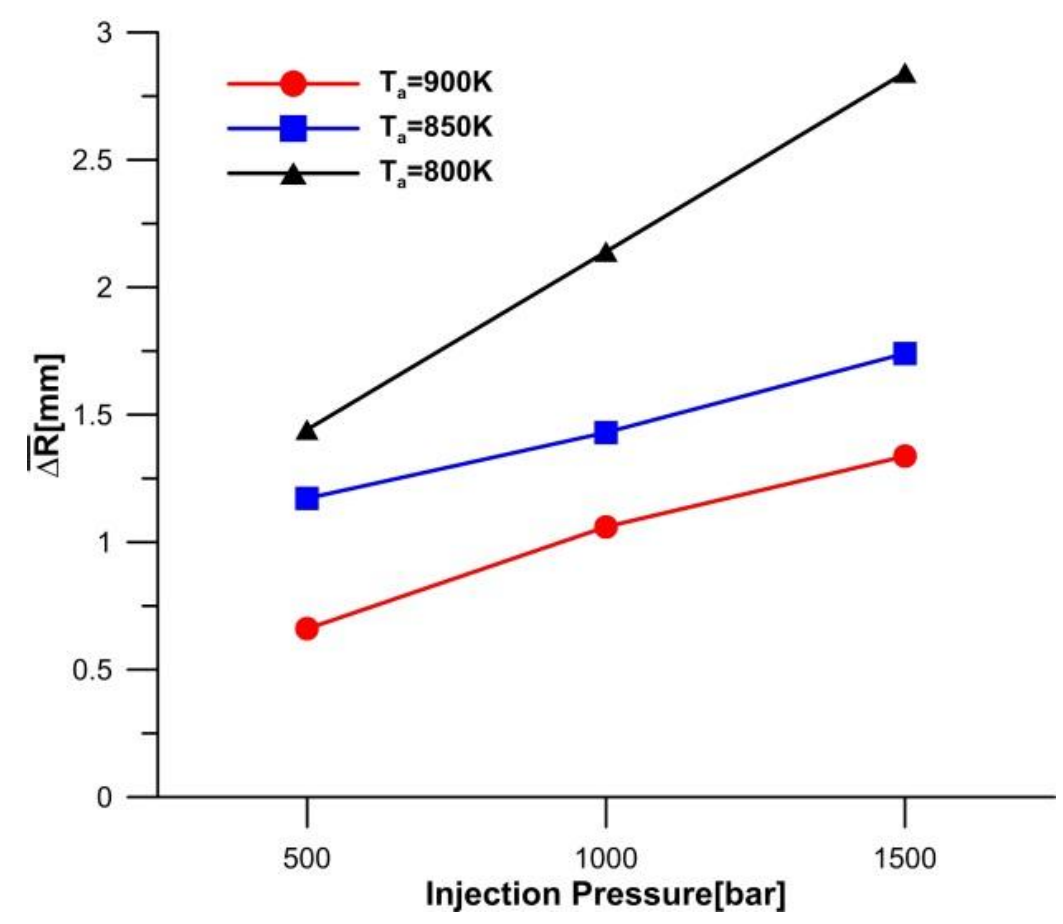

Fig.10. $\overline{\Delta \mathrm{R}}$ for parametric variation of injection pressure and ambient temperature. $\rho_{a}=22.8 \mathrm{~kg} / \mathrm{m}^{3}$, $O_{2} \%=15 \%$. 


\subsubsection{Effect of ambient density}

The temporal evolution of the penetration ratio and radial expansion when changing ambient density $\left(P_{i}=1,000\right.$ bar, $\left.T_{a}=900 \mathrm{~K}, O_{2} \%=15 \%\right)$ is shown in Fig.11 (left). There is an obvious increase in ignition delay and LOL with decreasing density. Thus, under the lowest density condition $\left(\rho_{a}=7.6\right.$ $\mathrm{kg} / \mathrm{m}^{3}$ ), a faster penetration and much longer ignition delay are observed, which prevents the observation of the acceleration and the quasi-steady phases because of the limitation of the optical access. It can be seen that the stabilization phase lasts longer with lower ambient density. At the quasi-steady phase, the penetration ratio increases with increasing density, which is different with previous work [12]. As for the radial difference, the observed evolution at $15.2 \mathrm{~kg} / \mathrm{m}^{3}$ condition is not as steady as for the other cases. This phenomenon can also be found when the injection pressure is at 1500 bar, which is not shown here. Probably, this was due to a more unstable flame development under low density condition.

The influence of the ambient density at two different injection pressures on the time-averaged $\overline{\mathrm{AR}}$ is shown in Fig.11 (right). As already commented, the time-averaged radial difference could not be obtained at $7.6 \mathrm{~kg} / \mathrm{m} 3$ because of the large LOL and ignition delay. As shown in Fig.11, the radial expansion decreases with higher ambient density, which is more sensitive at higher injection pressure
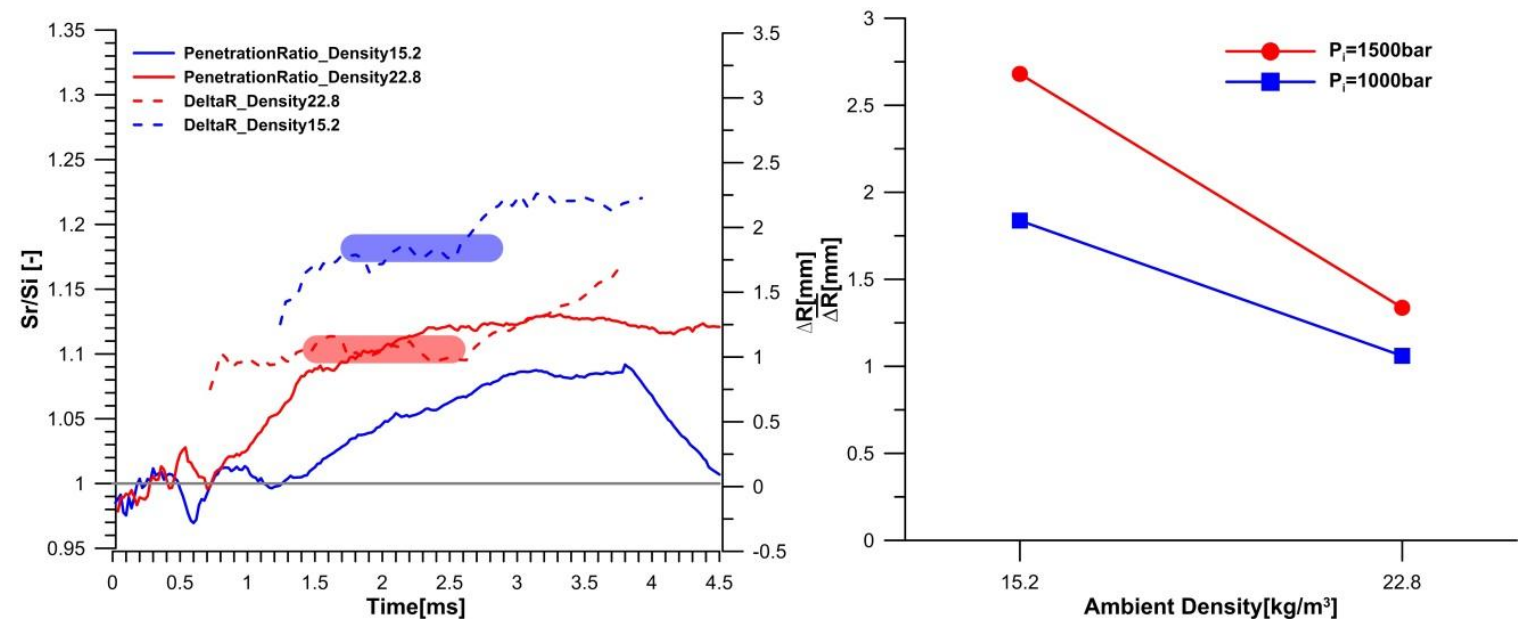

Fig.11. Temporal evolution of penetration ratio and radius expansion (left) and $\overline{\Delta R}$ (right) for a parametric variation of ambient density. $T_{a}=900 \mathrm{~K}, O_{2} \%=15 \%$.

\subsubsection{Effect of oxygen concentration}

Temporal evolution of the penetration ratio and radial expansion for an oxygen variation were investigated when keeping other parameters constant $\left(P_{l}=1,500 \mathrm{bar}, T_{a}=900 \mathrm{~K}, \rho_{a}=22.8 \mathrm{~kg} / \mathrm{m}^{3}\right)$. As shown in Fig.12 (left), when the oxygen concentration is $21 \%$, there is a sharp increase of the penetration ratio during the auto-ignition phase, as a consequence of the faster developing combustion process. The increasing rate and the peak value of the penetration ratio at the acceleration and quasi-steady phase always increase with higher oxygen concentration. However, the influence of oxygen on the radial expansion is not obvious, as shown in the temporal evolution of the radial difference (left) and the time-averaged $\overline{\Delta \mathrm{R}}$ (right). 

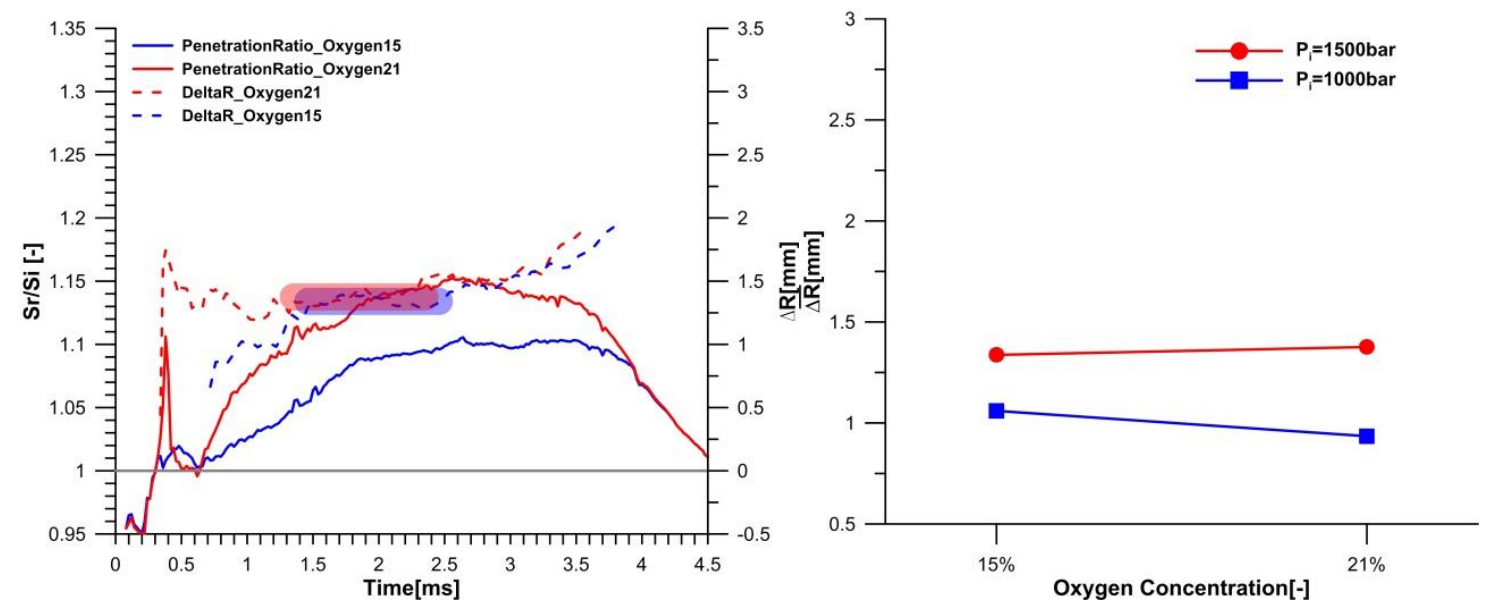

Fig.12. Temporal evolution of penetration ratio and radius expansion (left) and $\overline{\Delta R}$ (right) for a parametric variation of ambient density. $T_{a}=900 \mathrm{~K}, \rho_{a}=22.8 \mathrm{~kg} / \mathrm{m}^{3}$.

\subsubsection{Discussion}

From theprevious sections, two conclusions can be derived. First, there seems to be an inverse relationship between the radial increase and the penetration ratio. This has been observed for parametric variations of injection pressure, temperature and density. Only for the oxygen concentration is this relationship not observed. This can be explained to some extent in terms of simple momentum flux balances. From the point of view of a quasi-steady spray evolution, momentum flux at any cross section should be the same under inert or reacting conditions, and equal to the nozzle momentum flux. If by changing a parametric variable the radial increase is higher, the spray cross section is also higher, and therefore the expected average velocity should be lower, in agreement with the tip penetration ratio decrease. This argument is valid as long as density changes induced by transition to combustion are not very different among different operating conditions.

According to the previous reasoning, it would be important to evaluate which are the dependencies of the radius increase with the operating variables. The hypothesis has been made that the more fuel is available and therefore burnt at initial combustion stages, a stronger heat release and subsequent a higher lateral expansion is obtained.

Following this idea, the fuel mass downstream the lift-off length at the start of combustion (Mf) has been calculated for all experimental cases by means of 1D spray model [23][24]. The results are compared in Fig.13 with the radial increase. The blue points are the results of injection pressure and ambient temperature sweep, while the red and black points are the results of high oxygen concentration and low density cases respectively. According to this plot and the results in the above sections, it is quite apparent that a relationship exists between both variables, which to some extent validates the initial hypothesis. 


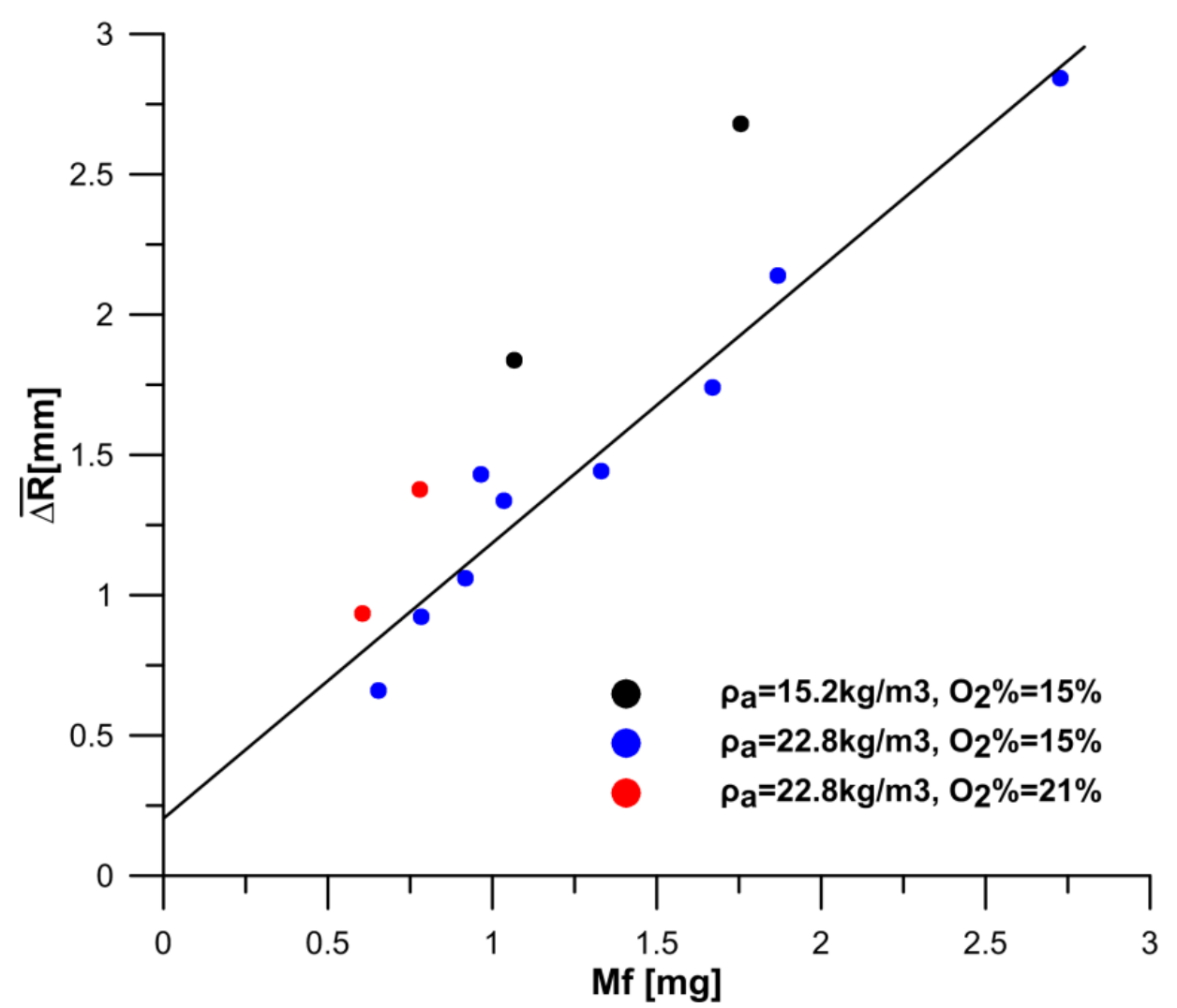

Fig.13. $\overline{\Delta \mathrm{R}}$ as a function of fuel mass from lift-off length to tip penetration at the start of combustion.

A clear linear relationship is observed in Fig. 13 between $\overline{\Delta R}$ and the fuel mass for the variation both injection pressure and ambient temperature. In other words, the difference of the expansion process caused by these two variables is governed by the amount of fuel mass in the igniting region (defined from the LOL until spray tip penetration). Injection pressure affects this characteristic fuel mass only in terms of the injection rate, as it has almost no effect on ignition delay $\left(P_{r}=1,500 \mathrm{bar}\right.$, $I D=0.46 \mathrm{~ms} ; P_{i}=1000 \mathrm{bar}, I D=0.49 \mathrm{~ms} ; P_{i}=1500 \mathrm{bar}, I D=0.52 \mathrm{~ms}$ ), while ambient gas temperature affects this characteristic fuel mass by the different ignition delay, with lower temperature leading to a longer ignition delay and therefore a higher injected mass. It must also be considered that both injection pressure and ambient temperature have a very limited effect on the spatial distribution of the equivalence ratio under inert conditions.

On the other hand, Fig. 13 shows that results for different ambient gas density and oxygen seem to follow a different although also linear trend. Both variables have a strong effect on the local equivalence ratio within the flame, either by modifying the mixture fraction distribution (density) or the oxygen content (oxygen). For a very similar fuel mass at the start of combustion, the equivalence ratio will certainly be different when changing any of both variables, compared to parametric variations of injection pressure and ambient temperature, which have a very limited effect on local equivalence ratio. As a result, resulting combustion temperatures and radial expansion effects will be different between oxygen/density parametric studies, and injection pressure/temperature ones.

The present analysis is only a first estimation of global effects during spray autoignition. More work is needed with detailed CFD simulations that can confirm the previous reasoning. 


\section{Conclusions}

In this study, Schlieren imaging technique has been performed to investigate the dynamics of the evolution of reacting sprays. All measurements were performed in a constant pressure flow vessel under a wide range of operating conditions. Spray penetration and radial width of the spray were investigated with particular focus to the axial and radial expansion of the spray occurring during the combustion. The most relevant observations and conclusions of this study are summarized below:

1) The temporal evolution of tip penetration, consistently with a previous work [12], can be divided into five phases: i) non-reacting, ii) auto-ignition expansion iii) stabilization, iv) acceleration and v) quasi-steady.

2) The radial width was calculated from the probability contours. From the analysis of the results it emerged that the shape of the reacting spray can be divided into three regions:

I. Quasi-steady inert: from nozzle tip to the Lift-off length.

II. Quasi-steady reacting: from lift-off length to the position where the contour stops increasing radially.

III. Transient: from the end of the previous zone to the spray tip.

3) After comparing of the radial width of reacting and inert spray at the same tip penetration, it was found that the contour at the quasi-steady reacting part is almost parallel to the corresponding part in the inert case. In other words, the radial contour of the reacting spray is simply the result of shifting the inert one by an approximately constant value.

4) After the analysis of axial and radial expansions under different test conditions the authors observed a relationship between these quantities and the amount of fuel burned during at the onset of combustion. As a consequence of that the following experimental observations have been explained as below:

a. The radial expansion increases with higher injection pressure and lower ambient gas temperature. It was found with constant ambient density and oxygen concentration, the time-averaged radial width difference increases linearly with fuel mas downstream the lift-off length at the onset of combustion. But higher injection pressure increases fuel mass before combustion by means of a higher mass flow rate, while lower temperature increases it by longer ignition delay.

b. The higher ambient gas density increases gas entrainment. However, this causes a reduction in the initial combustion portion because of the shorter ignition delay and lift-off length. As a consequence of that, the radial expansion decreases with higher ambient gas density.

c. Oxygen concentration has no significant effect on the radial expansion. Although the higher oxygen ratio can lead to a leaner equivalence ratio for combustion, it is compensated by the shorter ignition delay which reduces the fuel mass during the ignition delay. 


\section{Acknowledgements}

Authors acknowledge that some equipment used in this work has been partially supported by FEDER project funds (FEDER - ICTS - 2012 - 06), framed in the operational program of unique scientific and technical infrastructure of the Ministry of Science and Innovation of Spain.

\section{References.}

[1] R. Kiplimo, E. Tomita, N. Kawahara, and S. Yokobe, Effects of spray impingement, injection parameters, and EGR on the combustion and emission characteristics of a PCCI Diesel engine, Applied Thermal Engineering 37 (2012) 165-175.

[2] R. Mobasheri, Z. Peng, and S. M. Mirsalim, Analysis the effect of advanced injection strategies on engine performance and pollutant emissions in a heavy duty DI-Diesel engine by CFD modeling, International Journal of Heat and Fluid Flow 33 (2012) 59-69

[3] Haifeng Liu, Zhaolei Zheng, Mingfa Yao and Peng Zhang, Influence of temperature and mixture stratification on $\mathrm{HCCl}$ combustion using chemiluminescence images and CFD analysis, Applied Thermal Engineering, 33-34(2012) 135-143

[4] Jesús Benajes, Santiago Molina, Antonio García, Eduardo Belarte, Michel Vanvolsem, An investigation on $\mathrm{RCCl}$ combustión in a heavy duty diésel engine using in-cylinder blending of diésel and gasoline fuels, Applied Thermal Engineering, 63(2014) 66-76

[5] J. D. Naber, Dennis L. Siebers, Effects of Gas Density and Vaporization on Penetration and Dispersion of Diesel Sprays, SAE Technical Paper, 960034 (1996)

[6] I. V. Roisman, L. Araneo, C. Tropea, Effect of ambient pressure on penetration of a Diesel spray. International journal of multiphase flow, 33:8 (2007) 904-920

[7] F. Payri, R. Payri, M. Bardi, M. Carreres, Engine combustion network: Influence of the gas properties on the spray penetration and spreading angle. Experimental Thermal and Fluid Science, 53 (2014) 236-243

[8] M. P. B. Musculus, K. Kattke, Entrainment waves in Diesel jets, SAE Technical Paper, 2009-01$1355(2009)$

[9] John E. Dec, A Conceptual Model of DI Diesel Combustion Based on Laser-Sheet Imaging, SAE Technical Paper, 970873 (1997)

[10]M. P. B. Musculus, P.C. Miles, L. M. Pickett, Conceptual models for partially premixed lowtemperature Diesel combustion, Progress in Energy and Combustion Science, 39.2 (2013) 246283

[11]D., Siebers, Chapter 5 in Flow and combustion in reciprocating engines. Springer, 2009

[12] Pickett, L.M., Hoogterp, L. Fundamental spray and combustion measurements of JP-8 at diesel conditions (2009) SAE International Journal of Commercial Vehicles, 1 (1), pp. 108-118.

[13] J. M. Desantes, J. V. Pastor, J. M. García-Oliver, F. J. Briceño, An experimental analysis on the evolution of the transient tip penetration in reacting Diesel sprays, Combustion and Flame, 161:8 (2014) 2137-2150

[14]Pickett, L.M., Kook, S., Williams, T.C. Visualization of diesel spray penetration, cool-flame, ignition, high-temperature combustion, and soot formation using high-speed imaging(2009) SAE International Journal of Engines, 2 (1), pp. 439-459 
[15] Engine Combustion Network webpage: http://www.sandia.gov/ecn (online). Last accessed December 2014

[16]M. Bardi, R. Payri, L. M. Malbec, G. Bruneaux, L. M. Pickett, J. Manin, T. Bazyn, C. Genzale, Engine combustion network: comparison of spray development, vaporization, and combustion in different combustion vessels, Atomization and Sprays, 22:10 (2012) 807-842

[17]R. Payri, J. M. García-Oliver, M. Bardi, J. Manin, Fuel temperature influence on Diesel sprays in inert and reacting conditions, Applied Thermal Engineering 35 (2012) 185-195

[18] M. Meijer, B. Somers, J. Johnson, J. Naber, S.-Y. Lee, L. M. Malbec, G. Bruneaux, L. M. Pickett, M. Bardi, R. Payri, T. Bazyn, Engine Combustion Network (ECN): Characterization and comparison of boundary conditions for different combustion vessels, Atomization and Sprays 22 (2012) 777-806

[19]J. Benajes, R. Payri, M. Bardi, P. Martí-Aldaraví, Experimental characterization of Diesel ignition and lift-off length using a single-hole ECN injector, Applied thermal engineering 58 (2013) 554563

[20] G. S. Settles, Schlieren and sadowgraph techniques: visualizing phenomena in transparent media (Experimental Fluid Mechanic), Springer Verlag, Heidelberg, Germany, 2001

[21] H. Kosaka, T. Aizawa , T. Kamimoto ,Two dimensional imaging of ignition and soot formation processes in a Diesel flame, International Journal of Engine Research, 6:1 (2005) 21-42

[22] L. M. Pickett, J. Manin, C. L.Genzale, D. L. Siebers, M. P. B. Musculus, C. A. Idicheria, Relationship between Diesel fuel spray vapor penetration/dispersion and local fuel mixture fraction, SAE Technical paper 2011-01-0686, (2011)

[23] J.V. Pastor, J.J. López, J.M. García, J.M. Pastor, A 1D Model for the Description of MixingControlled Inert Diesel Sprays, Fuel, 87 (2008) 2871-2885

[24]J.V. Pastor, J.J. López, J.M. García, J.M. Pastor, A 1D Model for the Description of MixingControlled Reacting Diesel Sprays, Combustion and Flame 156 (2009) 234-249 


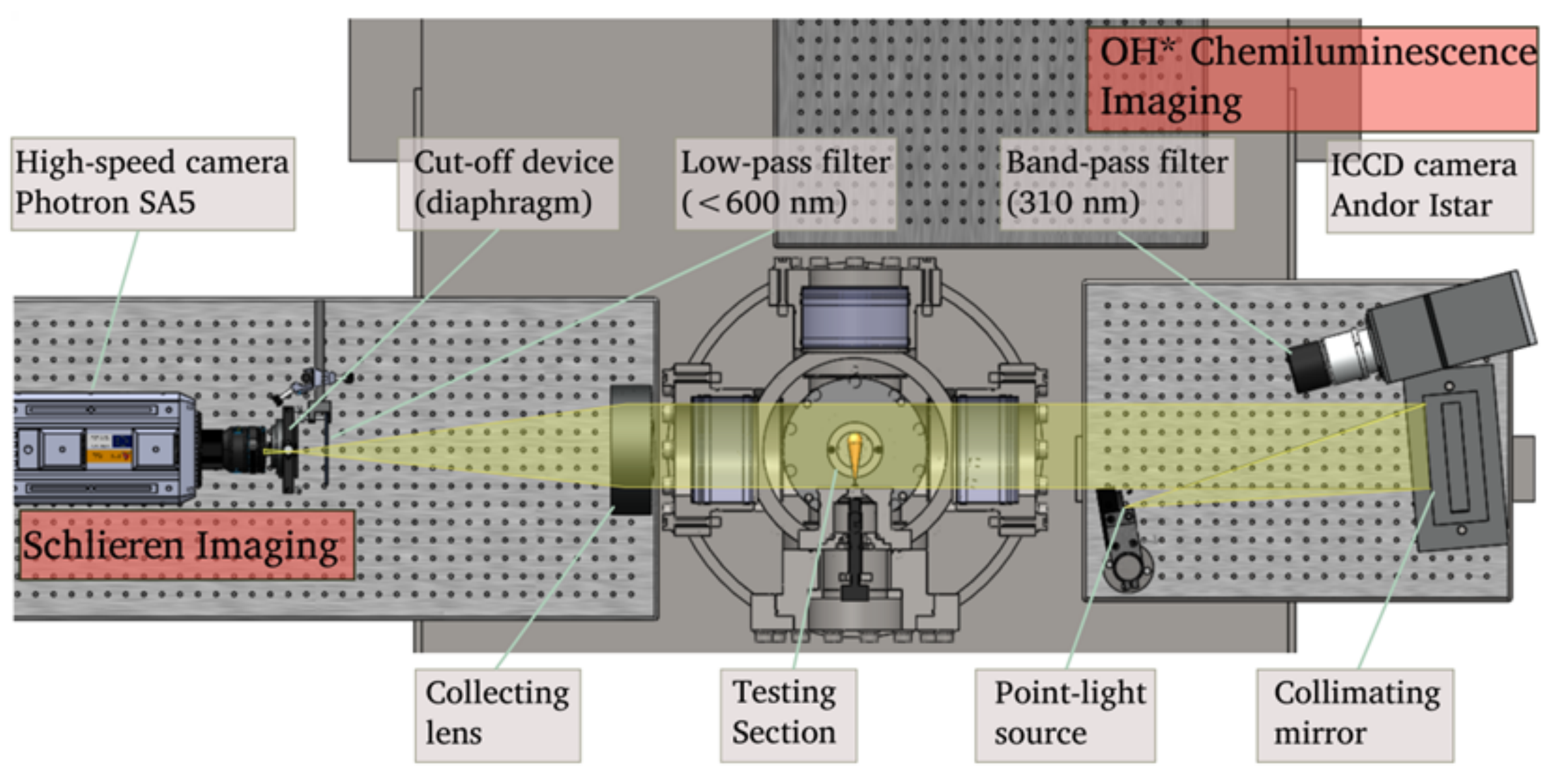


Schlieren images

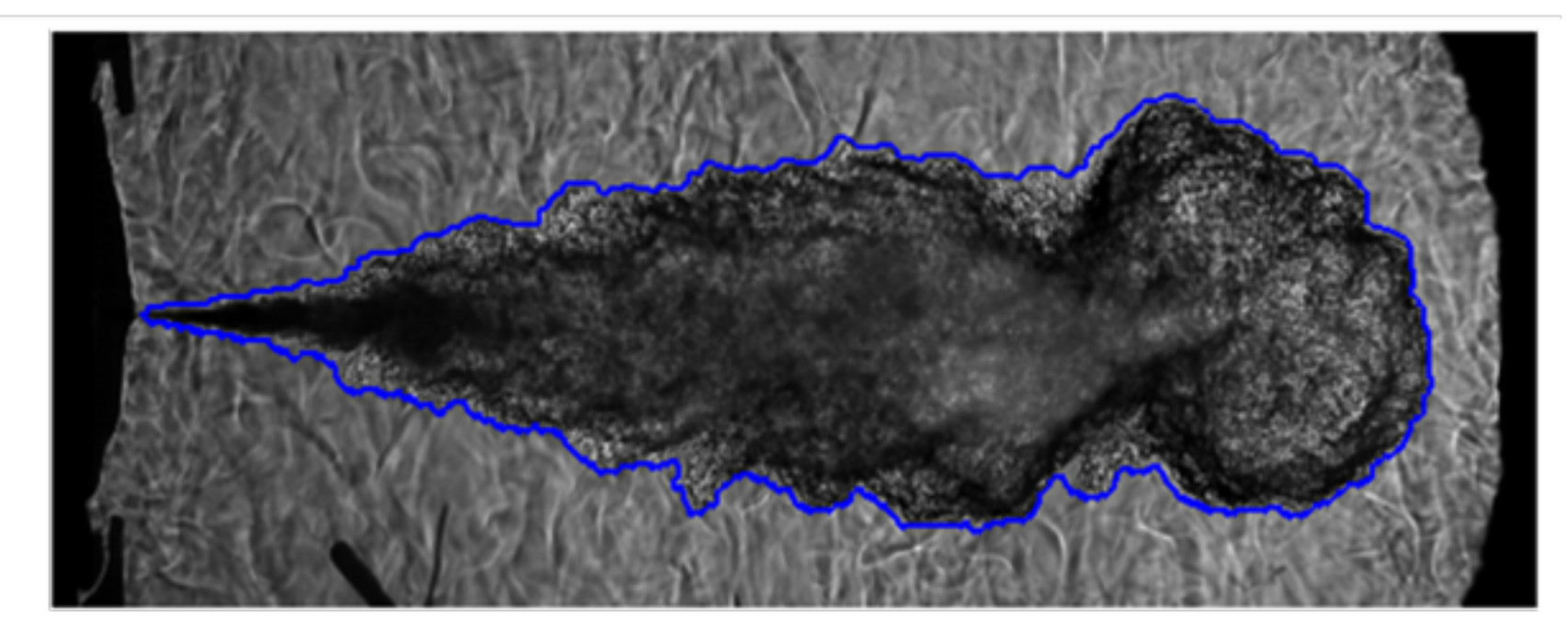

Binarization

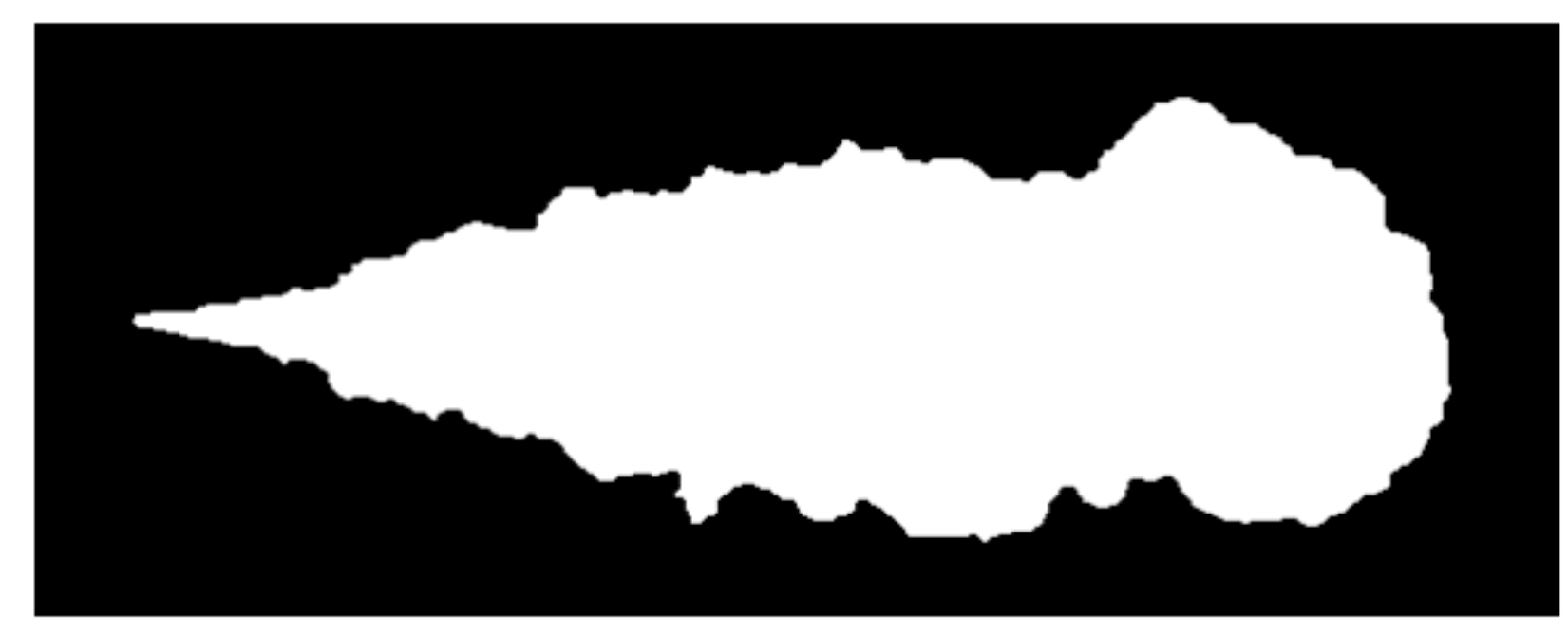

Rep.

\#1

$\vdots$

\#8

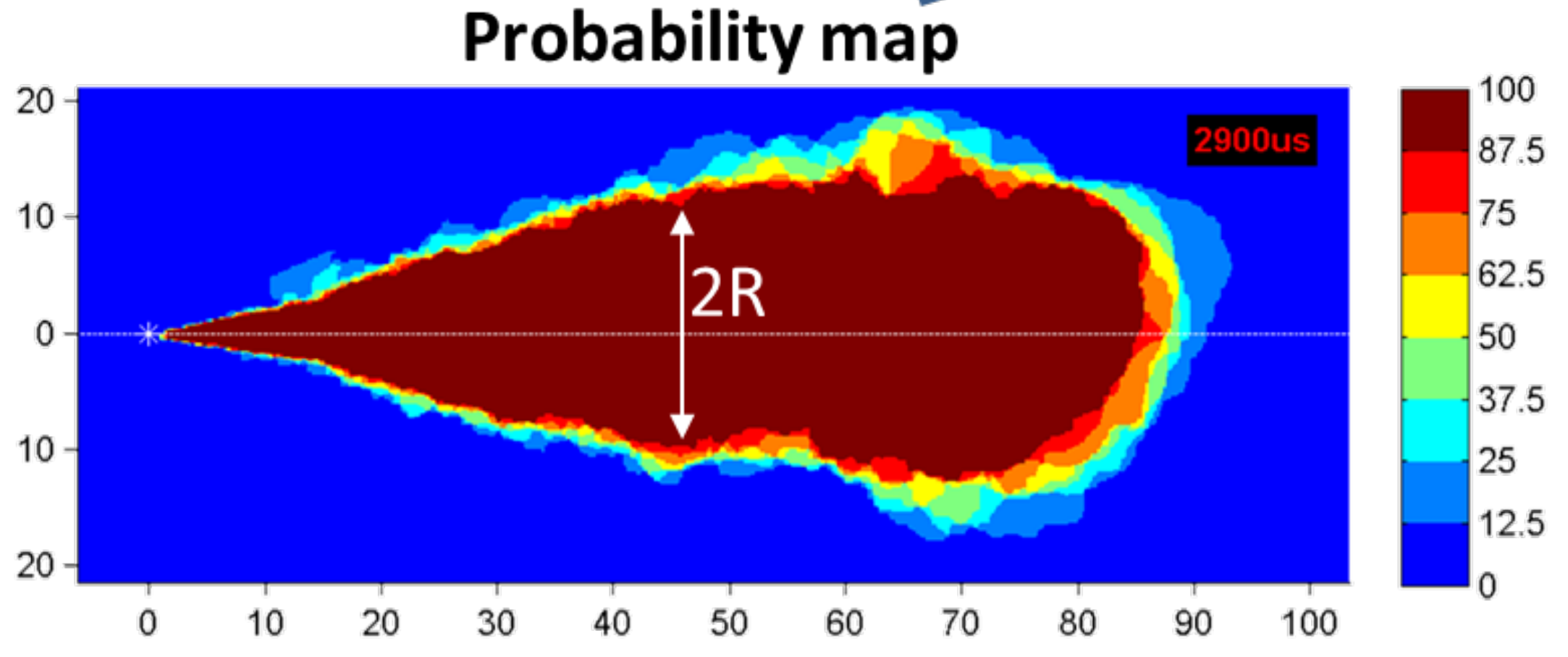




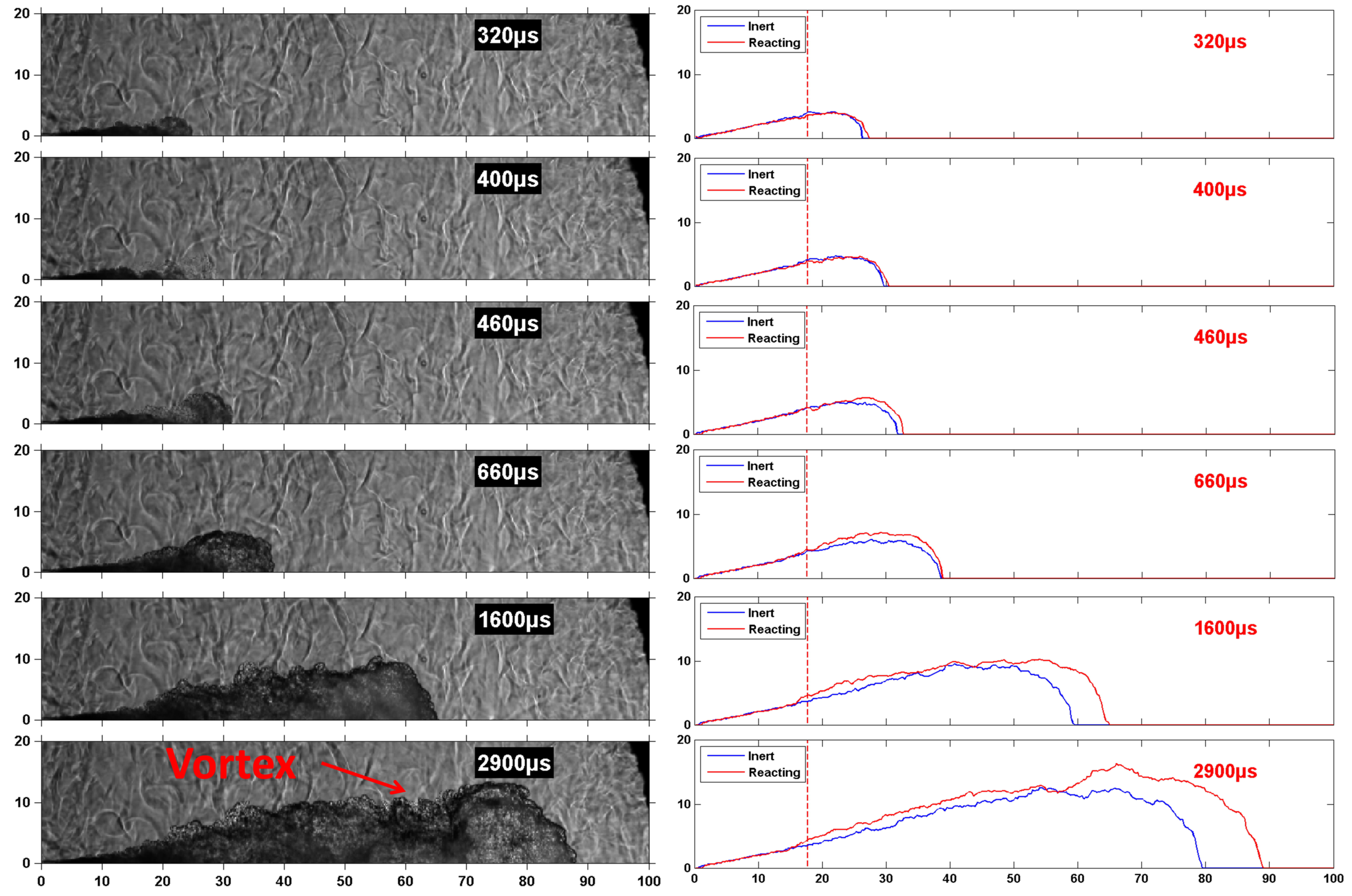


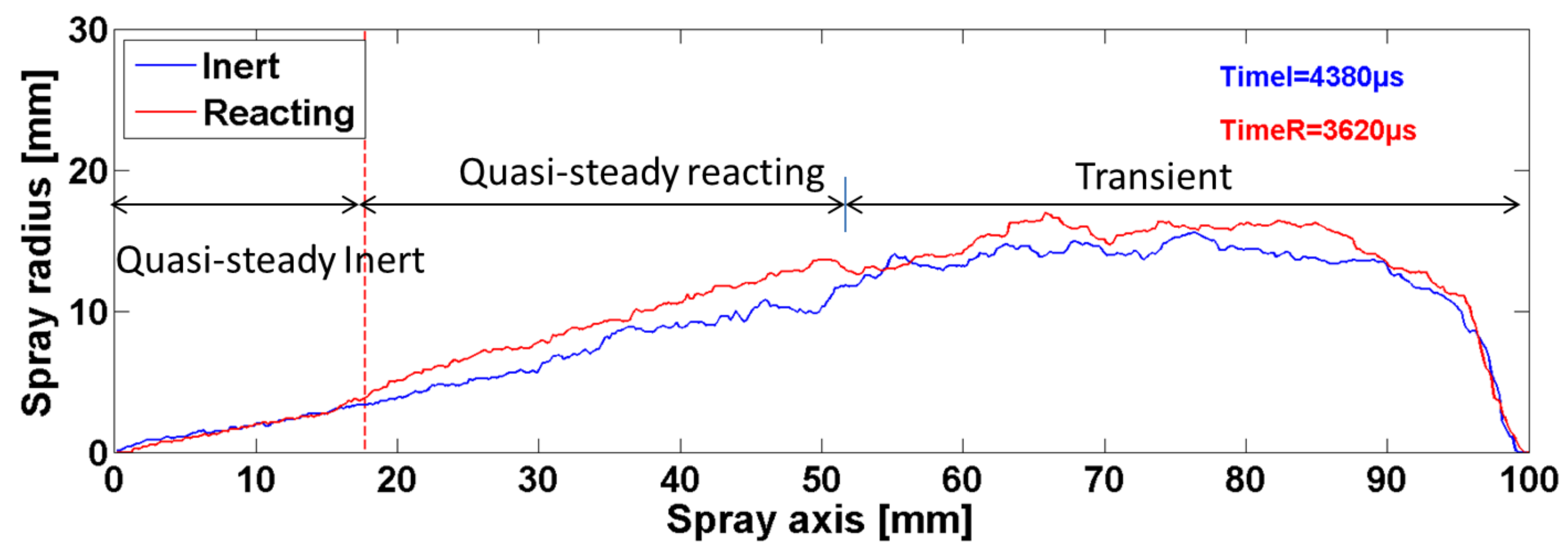



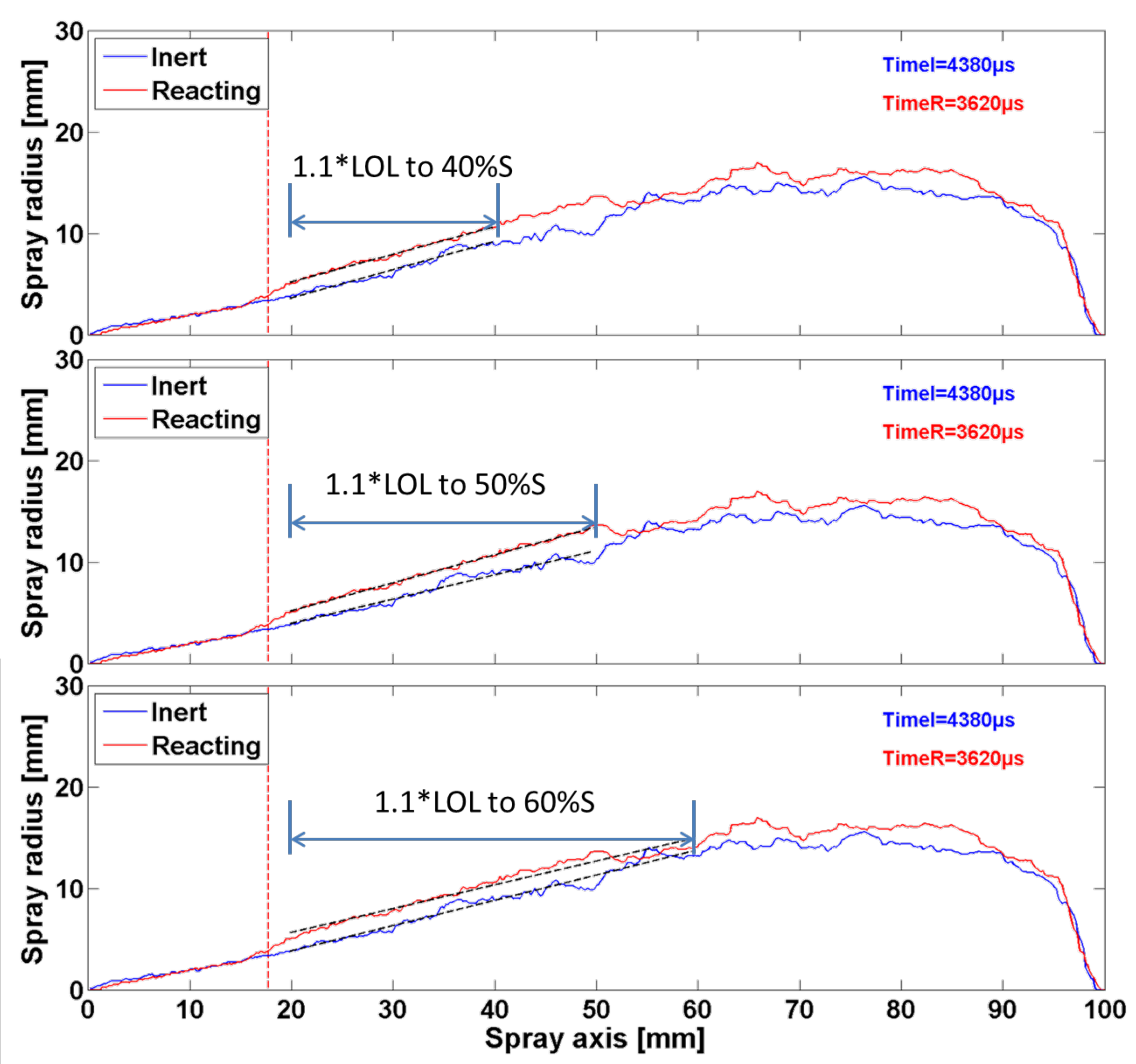


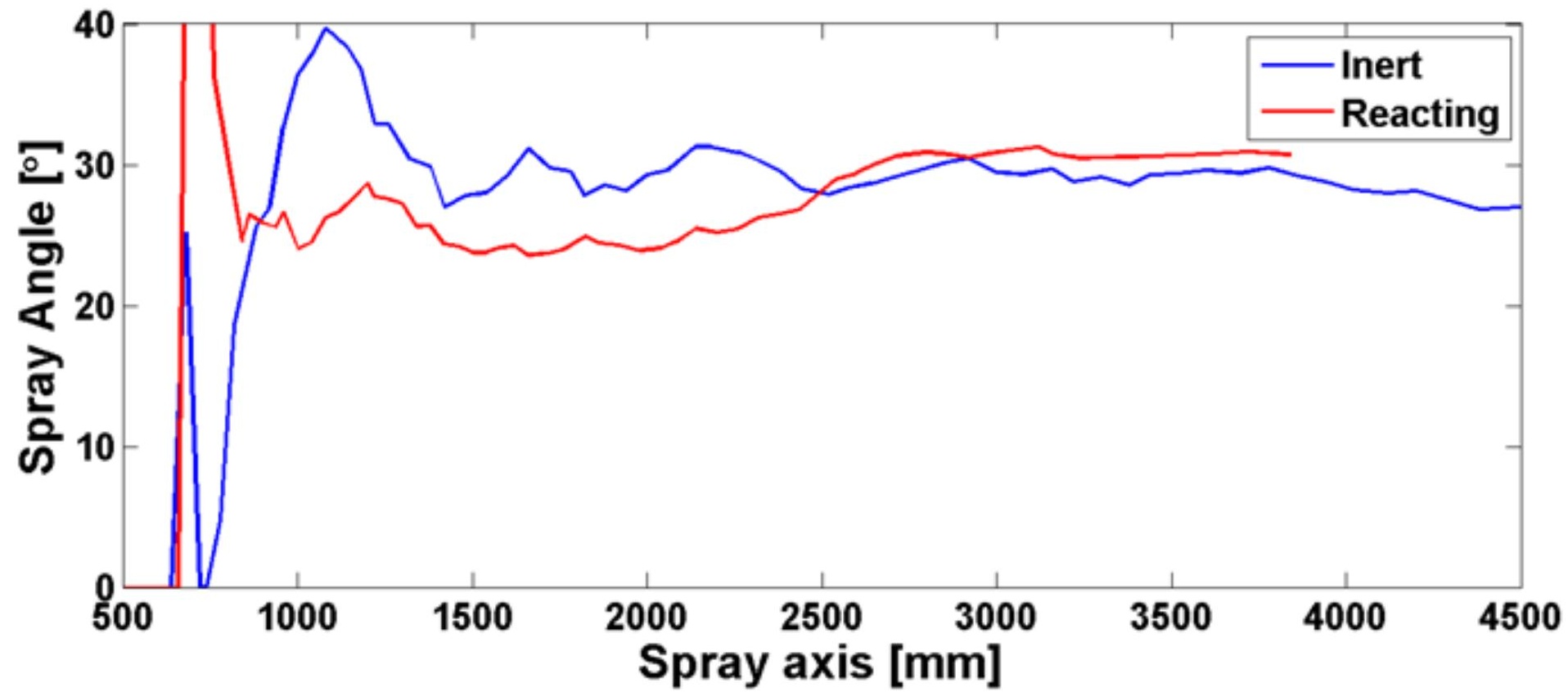




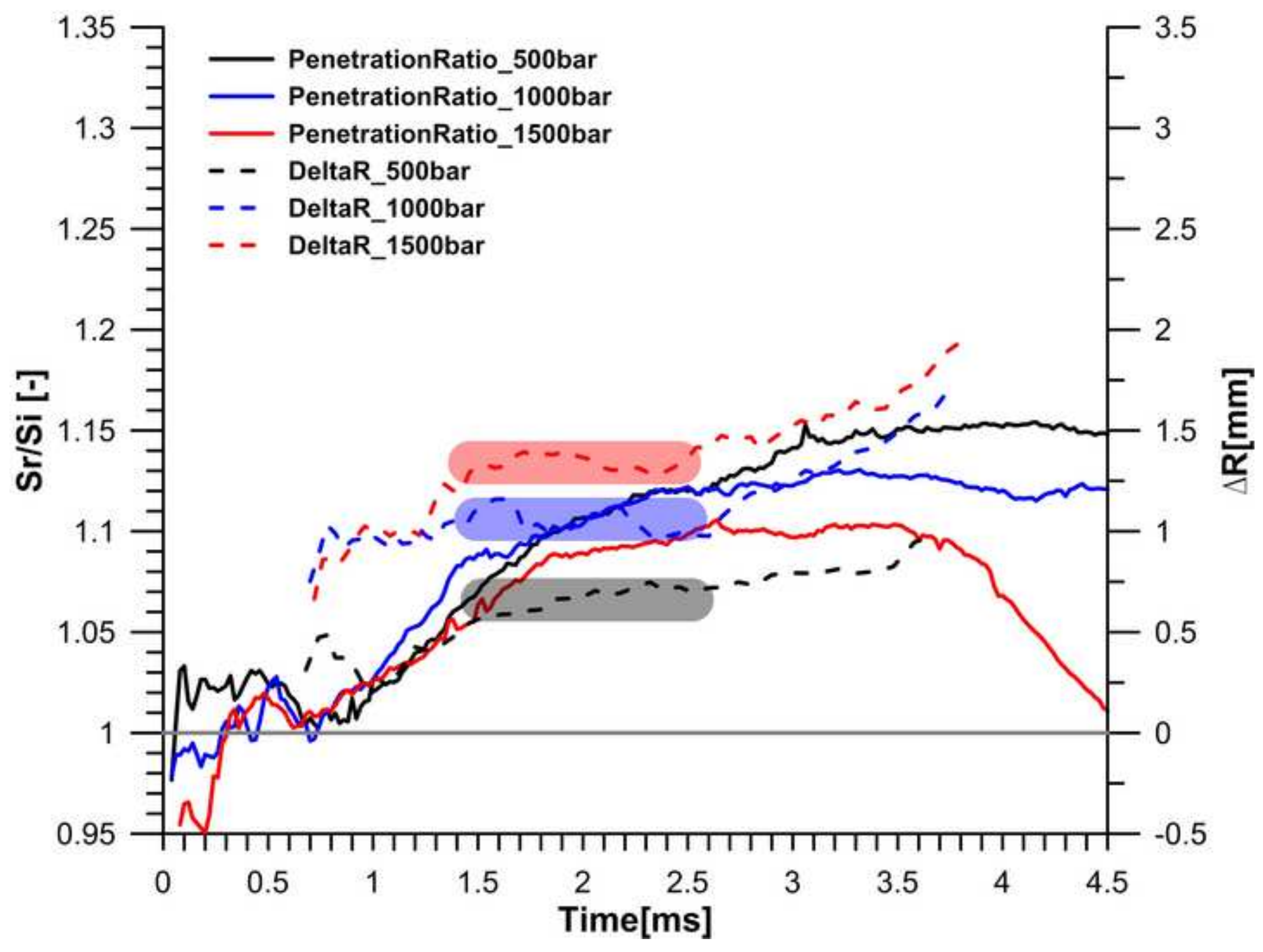




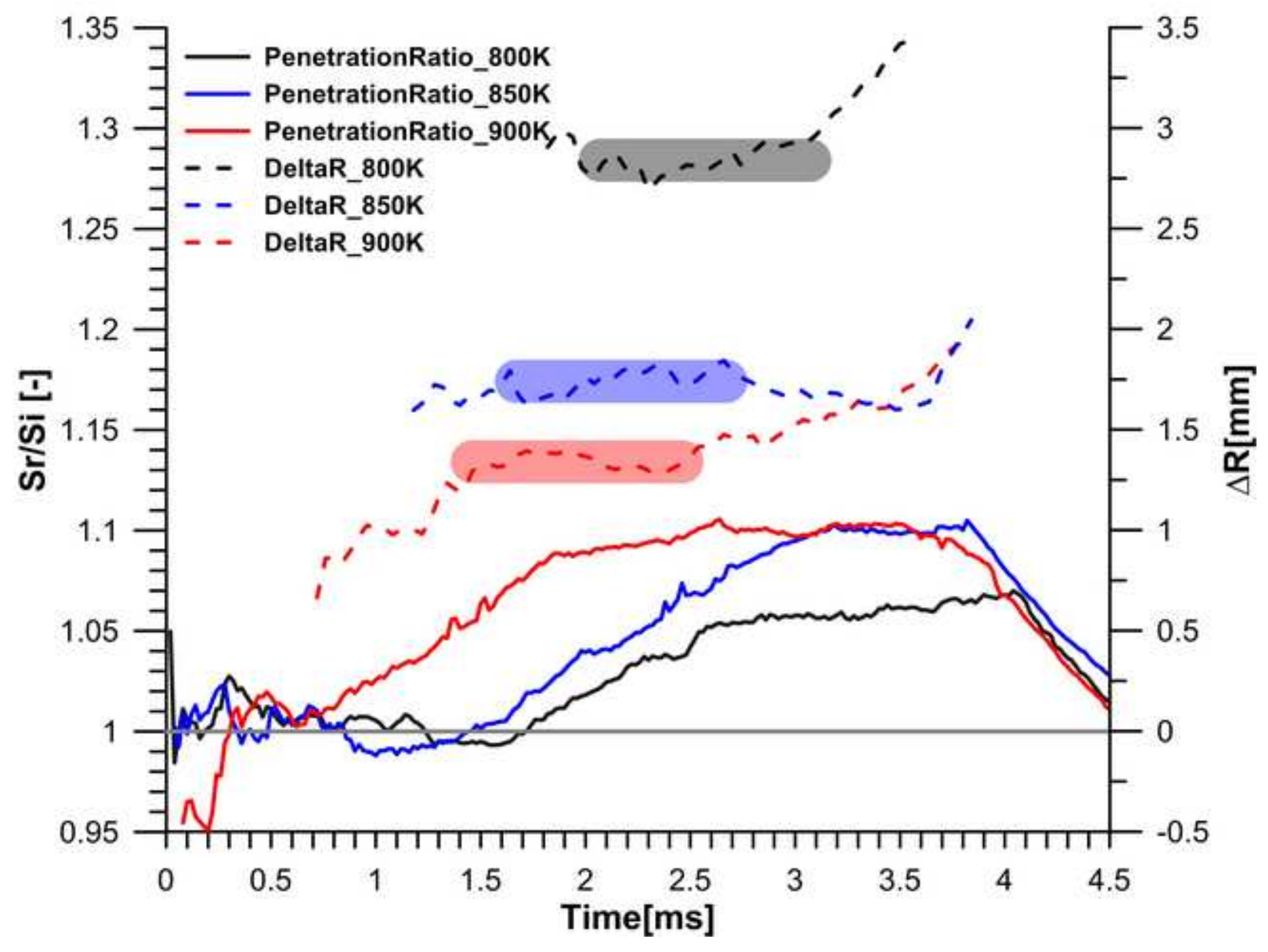




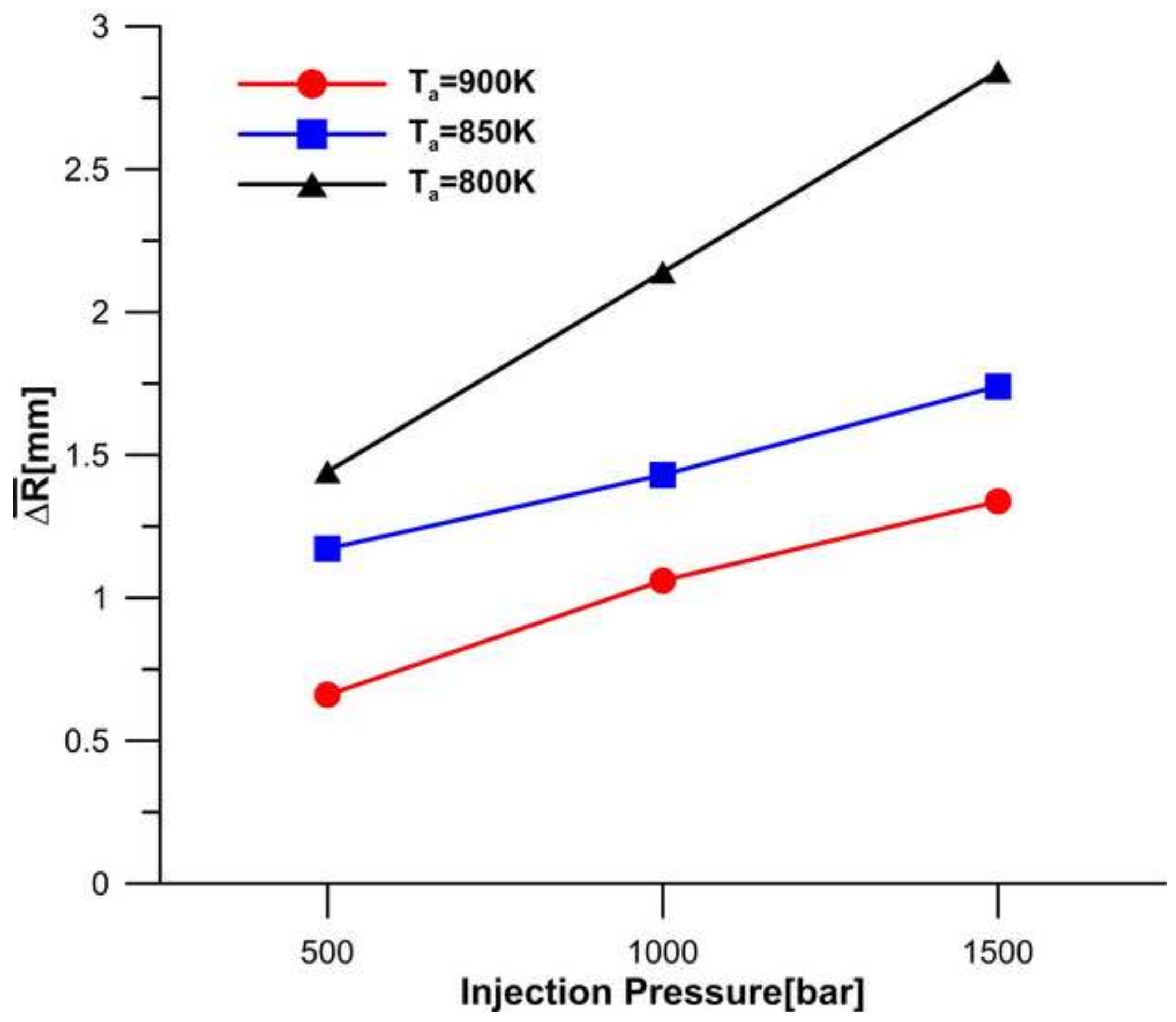




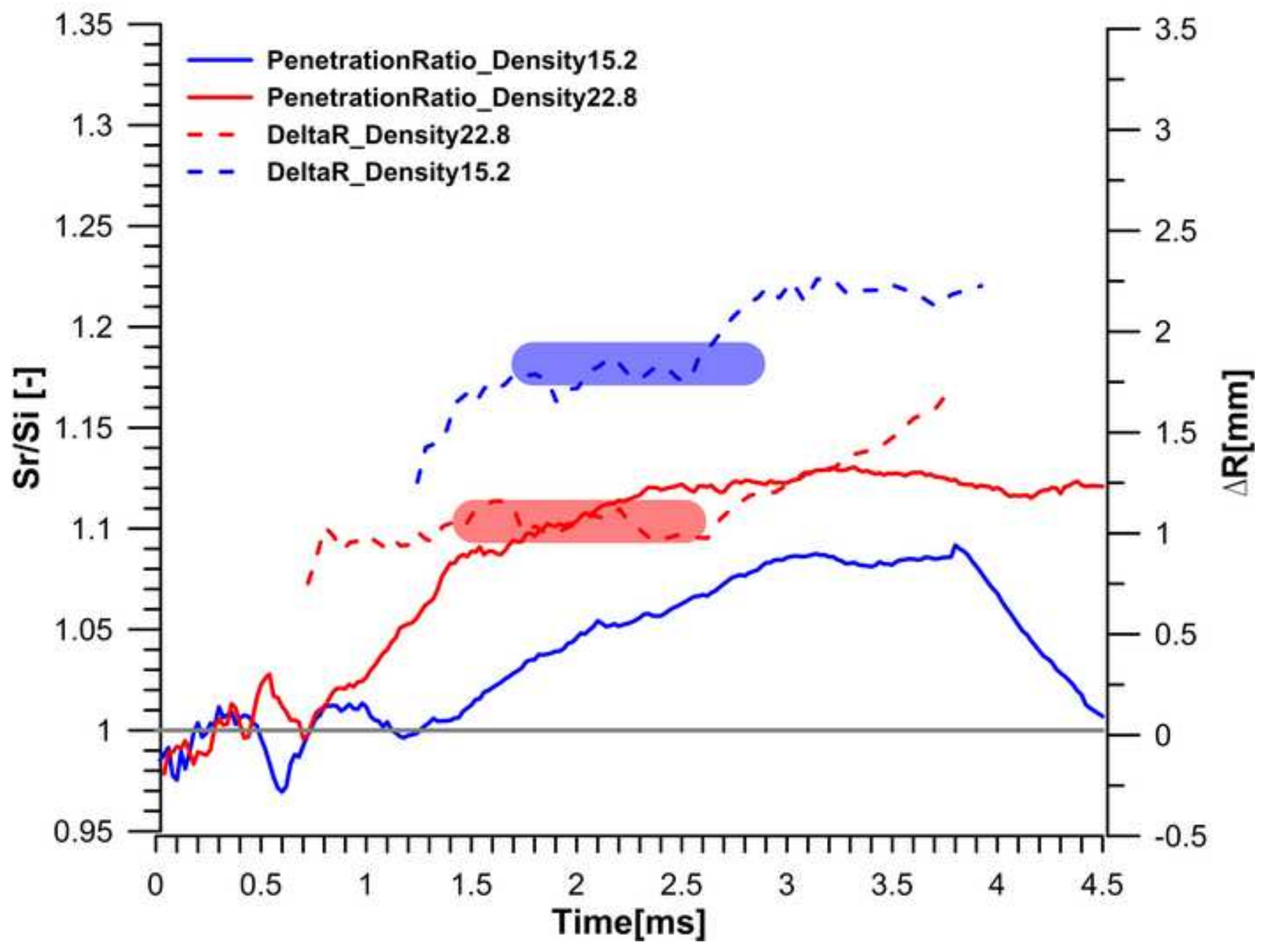




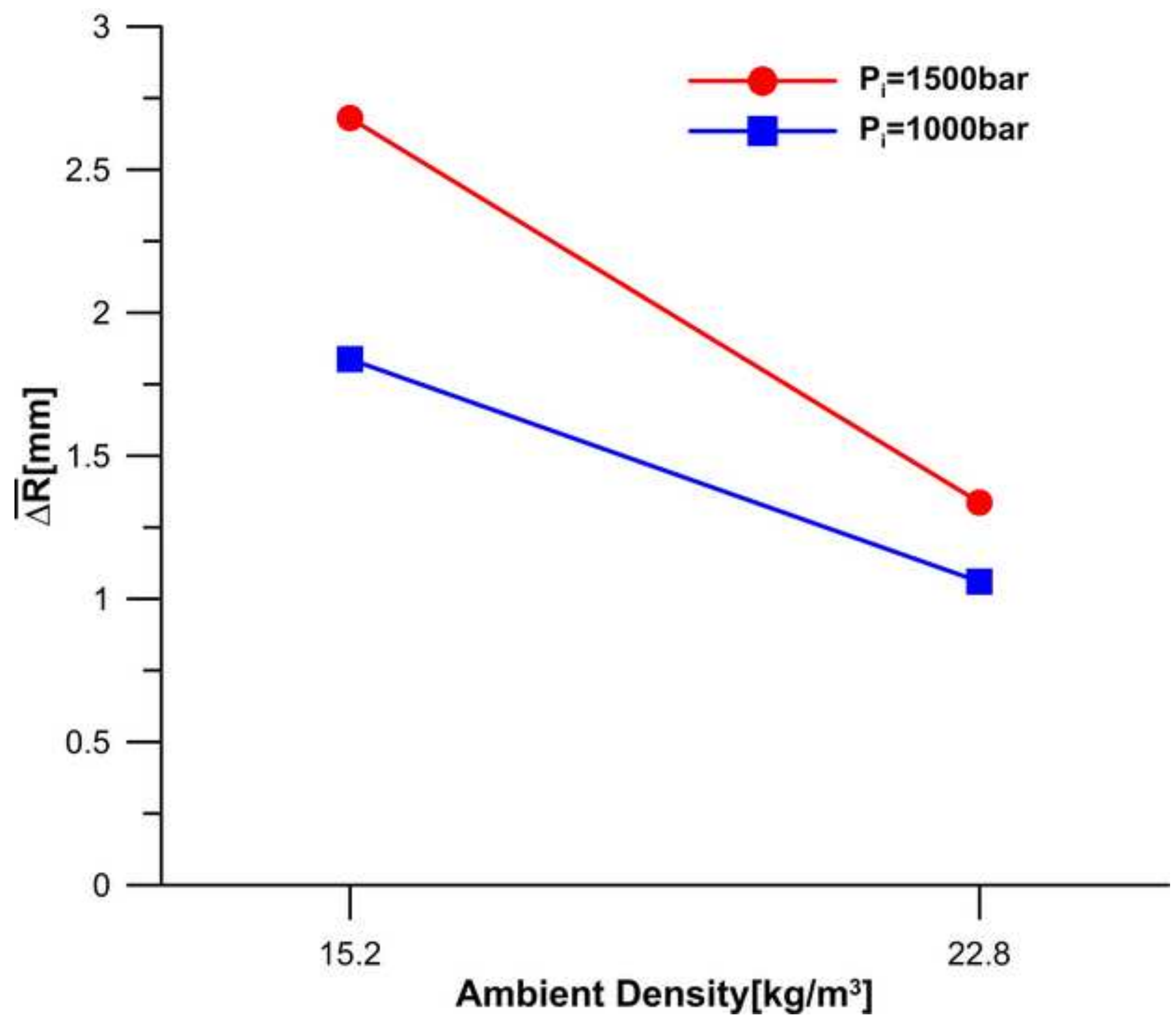




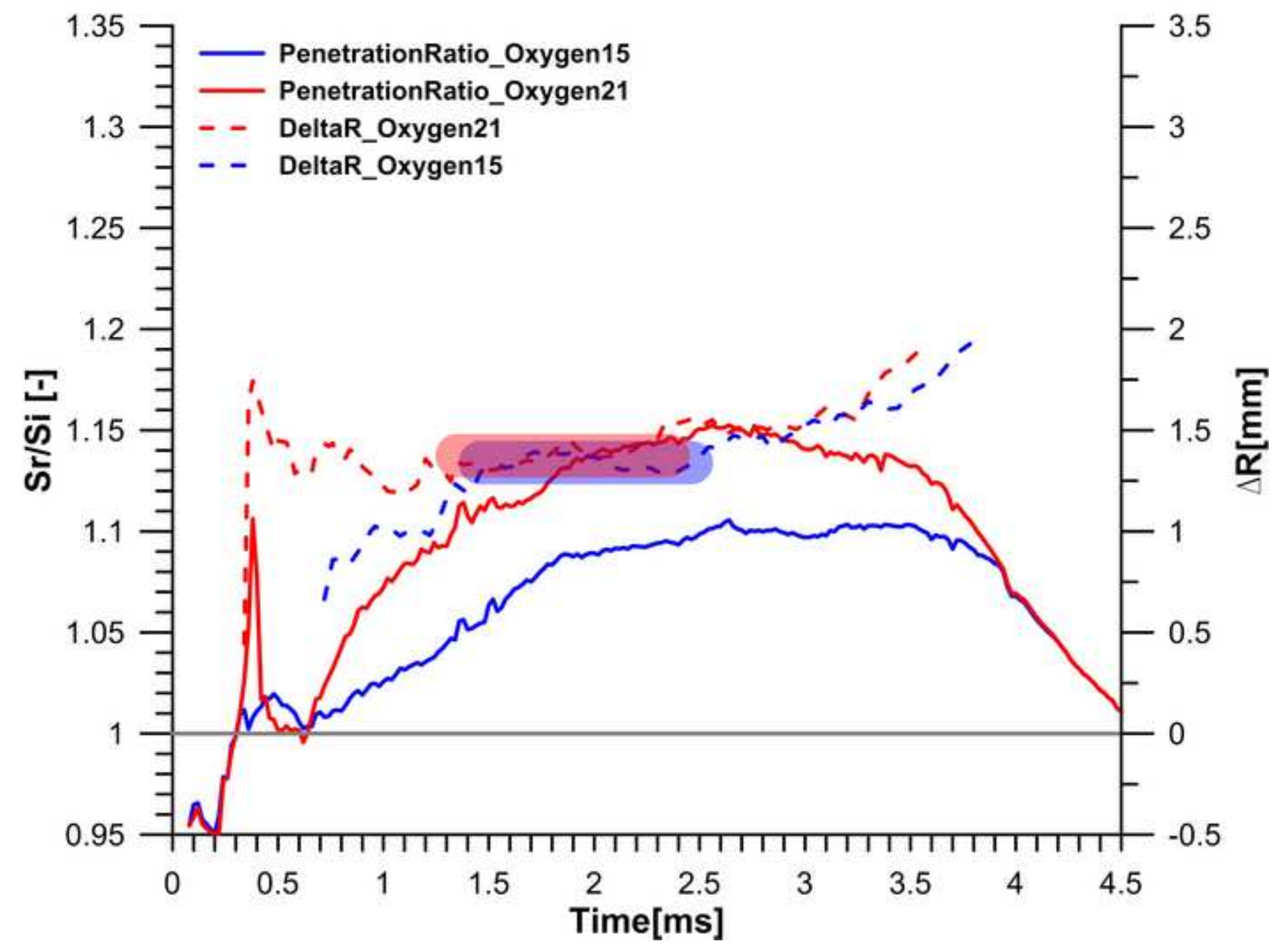




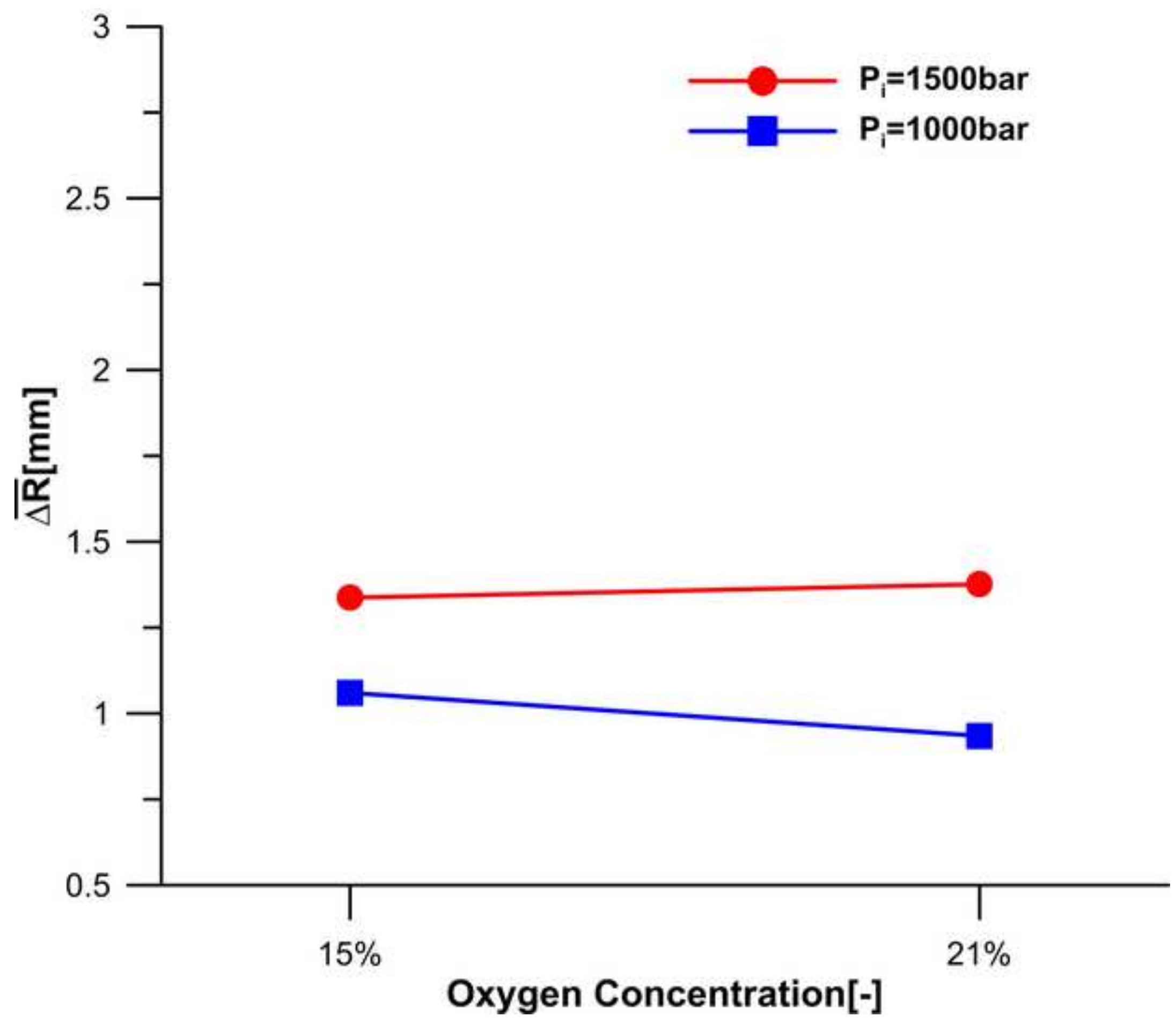




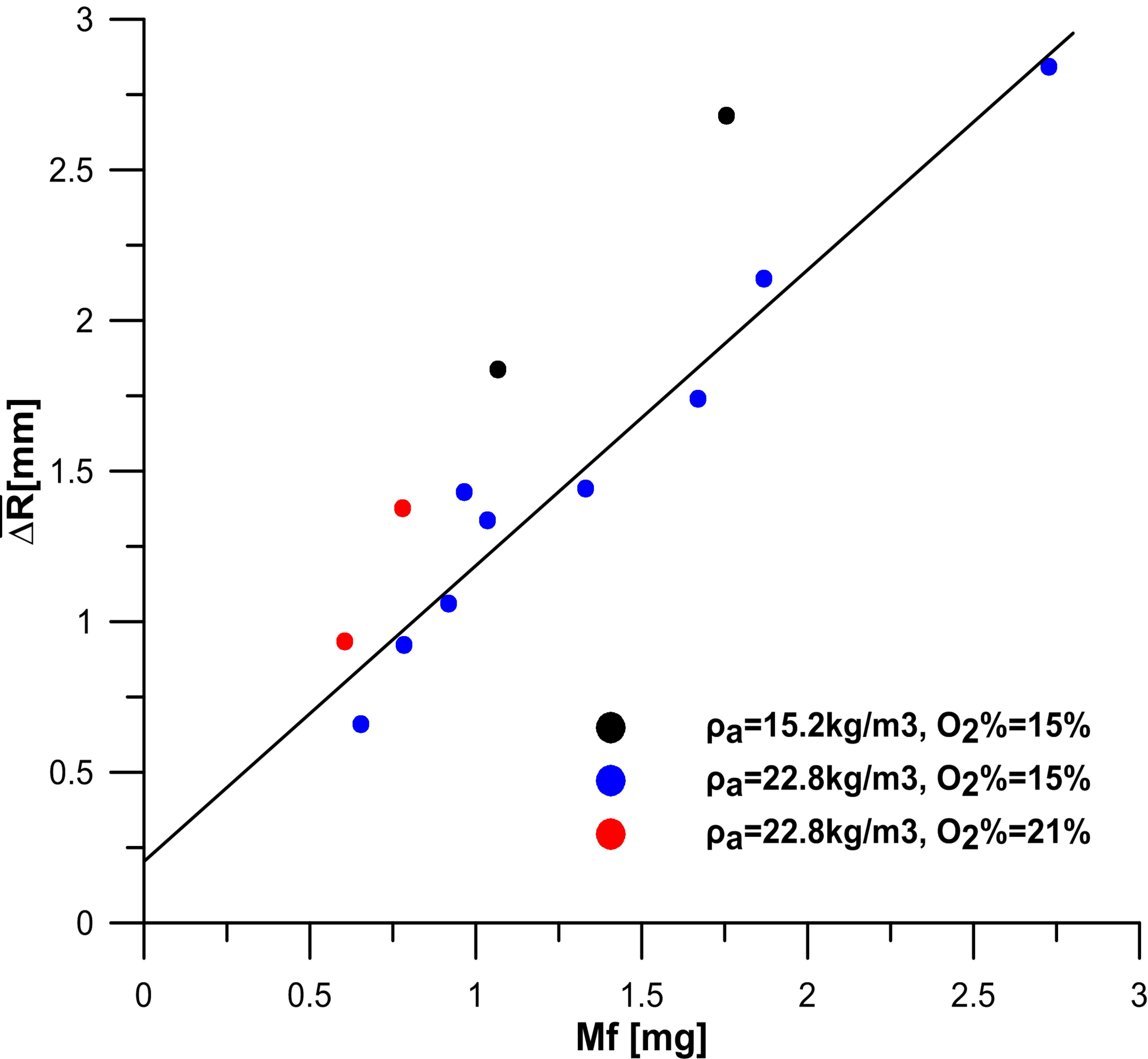

